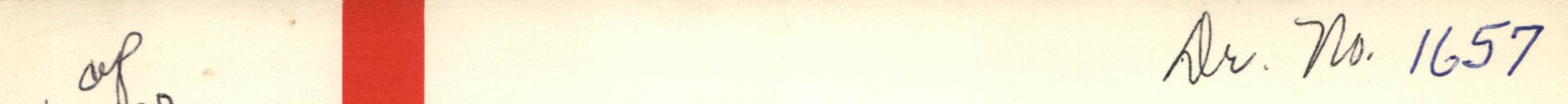

\title{
STABILITY OF \\ NUCLEAR REACTOR SYSTEMS HAVING TIME DELAYS
}

by

Kenneth Dermot Kearns

Department of Nuclear Engineering The University of Arizona Tucson, Arizona 85721

\section{June 1971}

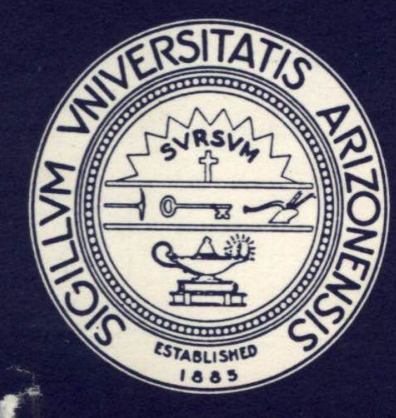

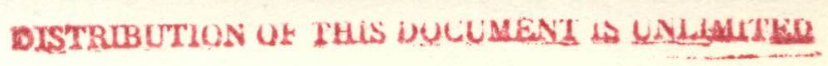

ENGINEERING EXPERIMENT STATION COLLEGE OF ENGINEERING

THE UNIVERSITY OF ARIZONA TUCSON, ARIZONA 


\section{DISCLAIMER}

This report was prepared as an account of work sponsored by an agency of the United States Government. Neither the United States Government nor any agency Thereof, nor any of their employees, makes any warranty, express or implied, or assumes any legal liability or responsibility for the accuracy, completeness, or usefulness of any information, apparatus, product, or process disclosed, or represents that its use would not infringe privately owned rights. Reference herein to any specific commercial product, process, or service by trade name, trademark, manufacturer, or otherwise does not necessarily constitute or imply its endorsement, recommendation, or favoring by the United States Government or any agency thereof. The views and opinions of authors expressed herein do not necessarily state or reflect those of the United States Government or any agency thereof. 


\section{DISCLAIMER}

Portions of this document may be illegible in electronic image products. Images are produced from the best available original document. 
STABILITY OF NUCLEAR REACTOR SYSTEMS

HAVING TIME DELAYS

by

Kenneth Dermot Kearns

Department of Nuclear Engineering
The University of Arizona
Tucsol, Arizona 85721

LEGAL NOTICE

This report was prepared as an account of work This report was prepared states Government. Neither sponsored by the United States Governmestomic Energy the United States nor the United States Atom, nor any of Commission, nor any of their employees, nor any June $1971 \quad \begin{aligned} & \text { their contractors, subcontractors, or the or assumes any } \\ & \text { makes any warranty, express or implied, or }\end{aligned}$ makes any warranty, expribility for the accuracy, com-

legal lability or sos of any information, apparatus, pleteness or usefulness of any informents that its use product or process disclosed, or represents.
would not infringe privately owned rights.

A dissertation submitted to the faculty of the Department of Nuclear Engineering in partial fulfillment of the requirements for the degree of Doctor of Philosophy in the Graduate College of the University of Arizona. Supported by the U. S. Atomic Energy Commission under Contract AT- (04-3) -670 .

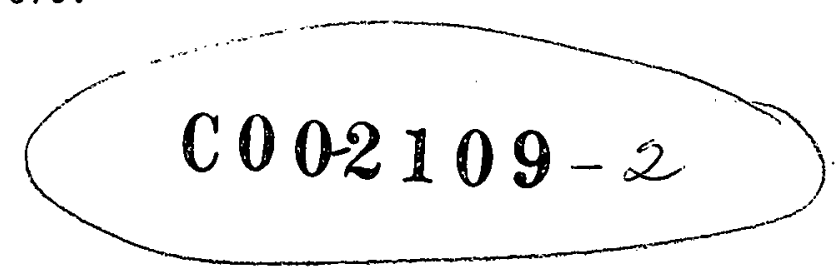




\section{ACKNOWLEDGMENTS}

The author is indebted to his advisor, Dr. Richard L. Brehm, for his direction and assistance during the performance of this research and to the United States Atomic Energy Commission for providing financial support for this study under Contract AT-(04-3)-670.

The author acknowledges the University of Arizona Computer Center for their generous grant of computer time and consulting services necessary in portions of this study.

The author wishes to thank the secretaries in the Department of Nuclear Engineering for their good nature and attention to detail in the preparation of this dissertation.

Finally, the author owes a special debt of gratitude to his wife, Linda, whose unfailing support was a tremendous incentive for the completion of this work. 
TABLE OF CONTENTS

Page

LIST OF ILLUSTRATIONS . . . . . . . . . . . . . . vi v vi

LIST OF TABLES . . . . . . . . . . . . . . . . . . . viii

ABSTRACT . . . . . . . . . . . . . . . . . . ix

1. INTRODUCTION . . . . . . . . . . . . . . . . . 1

2. EXISTING METHODS OF STABILITY ANALYSIS FOR SYSTEMS WITH

TIME DELAY . . . . . . . . . . . . . . . . . . 5

Approximations to the Time Delay . . . . . . . . 6

Linear Methods . . . . . . . . . . . . . . . 8

Approximation of the Exponential in the
Characteristic Equation .......... 13

Methods of Pontryagin ............. 15

The Nyquist Criterion . . . . . . . . . . 16

The Mikhailov Criterion ............ 20

The Modified Mikhailov Criterion . . . . . . . 22

Nonlinear Methods . . . . . . . . . . . . . 24

Summary of Existing Methods . . . . . . . . . 28

3. PARAMETRIC STABILITY ANALYSIS OF SYSTEMS WITH TIME DELAY • • 31

The Mikhailov Criterion for Delayed Systems . . . . . . 31

Critical Time Delays and the Existence of Regions
of Conditional Stability . . . . . . . . . . 40

Mapping Conditionally Stable Regions in System

4. APPLICATION OF THE PARAMETRIC STABILITY ANALYSIS TECHNIQUE

TO A THERMIONIC REACTOR MODEL . . . . . . . . . 55

The Thermionic Reactor Model . . . . . . . . . 55

The Characteristic Equation and the $\left(\omega^{2}\right)^{\mathrm{n}}$ Polynomial . . 70

The Parametric Stability Analysis . . . . . . . . . 73

Déscartes' Rule of Signs.............. 74

Sturm's Theorem ................ 81

The Stability Results ............. 83

Some Comparisons . . . . . . . . . . . . . . . . 98

The Zero-Delay Approximation ............ 98

The Infinite-Delay Approximation . . . . . . . . 98

The Taylor Series Approximation . . . . . . . 99 
TABLE OF CONTENTS-- Continued

Page

5. CONCLUSIONS . . . . . . . . . . . . . . . . 103

APPENDIX A: DEFINITIONS OF SYMBOLS IN CHAPTER 4 . . . . . 107

APPENDIX B: DEFINITIONS OF $\mathrm{C}_{i}$ CONSTANTS . . . . . . . • 111

APPENDIX C: DEFINITIONS OF $\mathrm{F}_{i}$ CONSTANTS . . . . . . . . 113

REFERENCES .................... . 115 
LIST OF ILLUSTRATIONS

Figure

Page

$2.1 \Gamma$-Contour in S-Plane . . . . . . . . . . . . . 18

2.2 Nyquist Diagram. $\tau=0.4$. . . . . . . . . . 19

2.3 Nyquist Diagram. $\tau=0.8$. . . . . . . . . . . . 19

2.4 Mikhailov Curves . . . . . . . . . . . . . . 23

2.5 Modified Silikhailov Diagrams . . . . . . . . . . 25

3.1 Closed Contour C . . . . . . . . . . . . . . 34

3.2 Example of Auxiliary Curve and Unit Circle . . . . . . 43

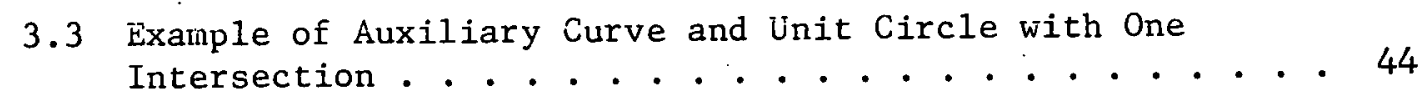

3.4 Unstable Miknailov. Curves for $\tau$ on Either Side of $\tau_{k l} \cdot{ }^{*} 45$

3.5 Example of Auxiliary Curve and Unit Circle with Two
Intersections . . . . . . . . . . . . . 47

4.1 Typical Representation of Series-Connected In-Core
Thermionic Diode in "Flashlight" Configuration . . . . 57

4.2 Thermionic Reactor System Schematic . . . . . . . . 58

4.3 Emitter Heat Flow versus Emitter Temperature . . . . . . 62

4.4 Schematic Representation of "Two-Well-Stirred-Tanks"
Approximation . . . . . . . . . . . . . 64

4.5 Results of Déscartes' Rule of Signs . . . . . . . . . 78

4.6 Zero-Delay Stability Results . . . . . . . . . . 80

4.7 Superimposed Results of Déscartes and Zero-Delay
Analysis . . . . . . . . . . . . . . . . 82

4.8 Results of Sturm's Sequence . . . . . . . . . . 84

4.9 Regions of Stability for Thermionic Reactor Model . . . 85 


\section{LIST OF ILLUSTRATIONS--Continued}

Figure

4.10 Locations of Reference Points and Reference Lines . . .

4.11 Modified Mikhailov Curves at RP-1. $\tau=1 \mathrm{sec}$. $\tau=10$ sec. ................ 88

4.12 Modified Mikhailov Curve at RP-1. $\tau=550 \mathrm{sec}$. . . . 89

4.13 Modified Mikhailov Curve at RP-2. $\tau=1 \mathrm{sec} . \quad . . . .90$

4.14 Modified Mikhailov Curve at $\mathrm{RP}-3 . \tau=10 \mathrm{sec} . . . . .90$

$4.15 \tau_{\text {crit-f }}$ versus $\gamma_{1}$ on $\mathrm{RL}_{-1}$. . . . . . . . . . . . . . 92

4.16 Modified Mikhailov Curves at RP-4. $\tau=10$ sec. $\tau=75 \mathrm{sec} . . . . . . . . . . . . . . . . .994$

4.17 Modified Mikhailov Curve at RP-4. $\tau=275 \mathrm{sec} . \quad$. . . 95

4.18 Modified Mikhailov Curve at RP-4. $\tau=375 \mathrm{sec}$. . . . . 96

4.19 Infinite-Delay Stability Result . . . . . . . . 100

4.20 Comparison of Exact Analysis and Taylor Series Approximations at RP-1 .. . . . . . . . . . 101 


\section{LIST OF TABLES}

Table

$2.1 \mathrm{X}(\omega)$ and $\mathrm{Y}(\omega)$ for Mikhailov Curves ........... 21

4.1 Equilibrium Conditions and System Parameters . . . . . . 75

4.2 Values of $\mathrm{C}_{i}$ and $\mathrm{F}_{i}$ for Example . . . . . . . . 76

4.3 Standard Forms of $\mathrm{A}_{i}$. . . . . . . . . . . . 77 


\section{ABSTRACT}

A technique based on the Mikhailov stability criterion has been developed to study the effects of time delays on the stability of linear systems. Specifically, the technique utilizes Déscartes' rule of signs and Sturm's theorem to determine regions within the system parameter space in which stability or instability is conditional on the value of the time delay. In these regions, the critical time delay indicating the stability boundary may also be determined.

The method is applied to a seventh-order, in-core thermionic reactor model containing two coolant time delays between the radiator and the core. Stability is found to depend on the sum of the two delays. The results of the analysis reveal the existence of conditional instabilities within physically possible operating regions of the párameter space. Within the conditionally stable regions, several critical time delays are calculated to indicate how stability depends on the value of the time delay.

A number of methods commonly used to study the stability of delayed systems are compared for their accuracy and ease of application. In addition, the results of the exact linear treatment are compared with three commonly applied approximations, all of which attempt to simplify the stability analysis. It is found that none of these approximations necessarily give conservative predictions of the exact stability zones. 
CHAPTER 1

INTRODUCTION

In the study of nuclear reactor systems, it is often necessary to predict the stability of systems whose mathematical models include terms that are delayed in time. These terms usually occur in the description of transport phenomena such as coolant lags. The purpose of this work is to develop and compare methods of determining the stability of delayed systems. More specifically, stability zones within the system parameter spaces are to be determined.

Upon perturbation of a system from equilibrium, a term with "pure" or "ideal" time delay will remain at its equilibrium value for some finite time, $\xi$, (the delay time) after which changes will occur. continuous1y but with lag. These effects are represented as state variables with delayed arguments

$x(t-\xi)$

Pure time delays appear in the description of distributed systems to characterize material and signal transport. Probably the most common of this kind is the description of coolant transport. In a thermionic reactor model (Brehm, Hetrick, Guppy, Kearns, and Schmidt 1968, p. $55 \mathrm{ff.}$ ), four delays are necessary in modeling the coolant transport between the reactor and the heat exchanger; and between the 
heat exchanger and the radiator. Similar lags appear in models of desalination plants (Ball 1967) and in nuclear power plants (Thompson and Beckerly 1964, p. 454).

The coupled-core reactor concept is another example of nuclear systems containing time delays (Chezem and Köhler 1967). In these models the source of neutrons in one core, which were born in another, are represented by a pure time delay of the form

$$
S(t-\xi)
$$

where $\xi$, the delay time, represents the neutron transit time between cores.

Not all of the time delay terms appear in the description of transport phenomena: They also occur between sensing and reacting of control devices, or in any instance where a human decision is necessary. Köhler (1969) uses a pure tịme delay to account for the delayed reactivity effect of the time required for a neutron to slow from fission to resonance energies.

Many so-called delayed effects which are prevalent in the modeling of nuclear systems are in fact not delayed in the ideal or pure sense. Upon perturbation of a system which is in equilibrium, deviations in these terms occur instantaneously although any appreciable change may develop much later. The mathematical description of delayed neutrons falls into this category. Since these effects present no special problems in stability analysis, our interest will be directed to systems which include pure time delays. 
It should be remembered that in many cases characterization by a pure time delay may be an approximation to a system more accurately described by a more complex set of equations. This is often necessary to make the system tractable. Occasionally this fact can be used advantageously when approximations to the time delay are necessary.

Ordinary differential equations which include terms that are delayed in time are members of a class termed differential-difference equations (Bellman and Cooke 1963). They are also called differencedifferential, differentials with deviating argument, and others. Although this class includes equations with variable time leads as well as lags, our interest here will be centered only on constant time delays. We will study time invariant, autonomous equations written in state variable notation.

The addition of time delays can often result in serious alterations to the stability of a system. Weaver (1963, p. 74) gives a simple example of a lag term driving a stable system unstable. Heath (1968) discusses a thermionic.system which as a result of effective time lags may be unstable in spite of negative temperature feedbacks.

In Chapter 2, we will discuss and compare the existing methods of stability analysis for systems with time delays. From this discussion we will select the Mikhailov criterion to be used as a basis of a technique to study stability in relation to system parameters. The development of this technique is given in Chapter 3. In Chapter 4, use is made of the technique to study the parametric stability of an example system; and in Chapter 5, conclusions from this work and suggestions for future work are given. 


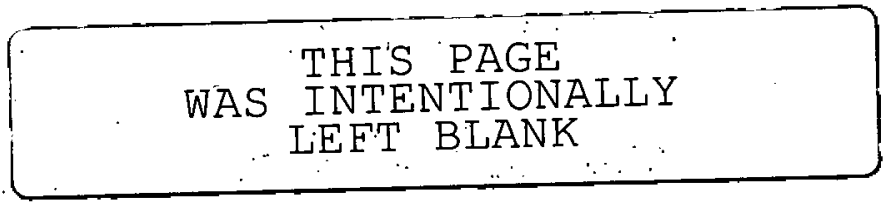


CHAPTER 2

\section{EXISTING METHODS OF STABILITY ANALYSIS FOR SYSTEMS WITH TIME DELAY}

In this chapter we will review a number of the principal techniques usually applied to systems with time delay, and we will discuss their applicability to the study of nuclear systems. These techniques will include several common approximations to the time delay terms, a number of methods applicable to linear or linearized systems, and a brief discussion of methods for nonlinear systems. Prior to this, we will begin to develop some of the notation which will appear throughout this work.

We will study time invariant, autonomous equations written in state variable notation. In matrix form, an $n^{\text {th }}$ order system with $m$ different time delays, $\xi_{i}$, will be written

$$
\underset{\vec{x}}{\dot{\vec{x}}}(t)=\mathrm{A}(\overrightarrow{\mathrm{x}}) \overrightarrow{\mathrm{x}}(\mathrm{t})+\sum_{i=1}^{\mathrm{m}} \mathrm{B}_{i}(\overrightarrow{\mathrm{x}}) \overrightarrow{\mathrm{x}}\left(t-\xi_{i}\right)
$$

where $\vec{x}$ is the vector of deviations from equilibrium of the state variables, and $A$ and $B_{i}$ are $n \times n$ matrices. The dot will denote the time derivative. Further,

$$
\xi_{\mathrm{m}} \geqslant \xi_{\mathrm{m}-1} \geqslant \ldots \geqslant \xi_{1}>0
$$

Generally, there will be many zero terms in A with the non-zero terms clustering around the diagonal exhibiting a band structure. This can 
be convenient for determining stability by computer techniques. Also, the $B_{1}$ matrices are normally very sparse.

Whereas the solution of ordinary differential equations displays a continuous dependence on the initial values of the variables at $t=0$, solutions of equations with delayed arguments continuously depend on initial function conditions

$$
\vec{x}(t)=\vec{g}(t) \quad-\xi_{m} \leqslant t \leqslant 0 .
$$

Since we are only interested in deviations from equilibrium, our initial functions will in many cases be

$$
\vec{x}(t)=\overrightarrow{0} \quad-\xi_{m} \leq t \leq 0 \text {. }
$$

Approximations to the Time Delay

In some situations certain approximations to the pure time delay terms may be justified. These approximations generally transform the system into a set of differential equations which have no-delayed terms and hence can be studied by conventional, more easily applied methods such as Routh or Root-Locus, or by simply solving for the roots of the characteristic polynomial and checking for positive real parts. Some of the techniques which are normally used to approximate time delays are discussed below.

Since we are considering only time delays in this work, and since for stability we are considering deviations from equilibrium, probably the simplest way of approximating the delayed terms is by letting them be zero. This in fact assumes that the time delay is much larger than the time constants of the system, implying that the delayed 
terms: never differ significantly from their equilibrium values. When these assumptions are justified, this technique can be very useful.

In his discussion of this particular approximation, El'sgol'ts (1966, p. 64) proves the following theorem for linear systems: - if the characteristic polynomial of the equation in which the delayed terms have been set to zero has at least one root with a positive real part, then, for a sufficiently large value of the smaliest delay, the system with delay is zonstable. Unfortunately, similar assertions to asymptotic stability can not be made without additional assumptions.

Another simplifying approximation which can be used is to neglect the delay constant thus leaving the system without delayed terms. This approach is justified when the delay is very short compared to the time constants of the system (e.g. very long prompt-neutron generation times and very short delays (Gage, Adler, and Powers 1966, p. 48)). In his discussion of this approximation, El'sgol!ts (1966, p. 61) proves the following theorem: if the set of equations in which the time delays have been set to zero is asymptotically stable, then for a sufficiently small value of the largest time delay, the unapproximated linear system is asymptotically stable. Similar conditions are true for unstable sys tems .

Unfortunately, in both of the above approximations, one must have knowledge about the size of the parameters before the simplifications can be made; and one can of ten be limited in the range of certain parameters if he has chosen to use one of these approximations (Kohler 1969). Clearly, these approximations are frequently too restrictive for a complete parametric stability analysis. 
A method which has been used with some success in approximating the delayed terms is by a Taylor series expanded about $t$ (Brehm, Hetrick, Guppy, Kearns, and Schmidt, 1968; p. 55 ff.; Forbes 1958, E1'sgo1'ts 1964, p.. 191).

$$
x(t-\xi)=x(t)-\xi \dot{x}(t) / 1 !+\xi^{2} \ddot{x}(t) / 2 !+\ldots+(-\xi)^{n} x^{n}(t) / n !+\ldots
$$

Because carrying more than two terms of the series will increase the order of the system, the expansion is usually truncated after two terms thus reducing the system model to a set of equations without delayed terms, but with the important advantage that the time delay constants remain among the parameters of the system. This allows a rough analysis of how the stability depends on the delay constants. Although the Taylor series is still an approximation which can lead to invalid results, it is nevertheless an important technique if used with care. In cases where the delayed terms are themselves approximations, it is possible that their further approximation by the methods discussed above may be just as representative of the physical nature of the system. In these cases the approximations are wholly justified.

\section{Linear Methods}

Of the methods normally applied to linear systems without delay, few can be extended to include delayed terms. This is due primarily to the change in form of the characteristic equation. This problem as well as approximations which alleviate it will be discussed in this section. 
Taking the Laplace transform of an $n^{\text {th }}$ order system without delays, performing the necessary algebra, and forming the integral for the inverse Laplace transform, all lead to a characteristic polynomial of order $\mathrm{n}$. The zeros of this polynomial are the poles of the integrand of the inverse Laplace transform closed at infinity. From Cauchy's residue theorem, it follows that the roots of the characteristic polynomial having positive real parts will result in growing exponentials in the solutions of the equations thus signifying an unstable system. Most of the methods applied to linear systems without delay are in some manner based on discovering whether or not the characteristic equation has roots with positive real parts. These methods can be subsumed under three categories (Wright and Kerlin 1968, p. 12):

1) methods "which determine the eigenvalues of the system matrix (roots of the characteristic polynomial), such as the reduction of the matrix to Hessenberg form and then using the QR transformation to determine the eigenvalues (Householder 1964, pp. 158, 197); 2) methods which operate on the coefficients of the characteristic polynomial such as the Routh criterion (Weaver 1963, p. 62);3) geometric methods such as the Mikhailov criterion (Popov 1962, p. 250) and the Nyquist criterion (Weaver 1963, p. 64).

As with ordinary systems, the stability of linear systems with delayed terms requires that all roots of the characteristic equation have non-positive real parts (Bellman and Cooke 1963, p. 115). However, for delayed systems, the characteristic equation contains exponentials resulting from the Laplace transforms of the delayed terms, 


$$
L[x(t-\xi)]=e^{-\xi s} x(s)
$$

Therefore, the characteristic equation of an $n^{\text {th }}$ order system is no longer a polynomial with at most $n$ distinct roots, but rather a transcendental equation with an infinite number of roots. This equation is normally termed a "quasi" or "exponential" polynomial. Because of the transcendental nature of the exponential polynomial, most of the above methods are precluded. A few, for example the geometric criteria, can be extended to include time delays. These techniques will be discussed later.

The linearized form of Eq. $(2-1)$ is written

$$
\dot{\vec{x}}(t)=\overrightarrow{A \vec{x}}(t)+\sum_{i=1}^{m} B_{i} \vec{x}\left(t-\xi_{i}\right)
$$

where $A$ and $B_{i}$ now represent constant $n \times n$ matrices: $A=\left[a_{j k}\right]$ and $B_{i}=\left[b_{i, j k}\right]$. Upon taking the Laplace transform of Eq. (2-7), the resulting characteristic determinant is of the form $M(s)=$ $\left|\begin{array}{cccc}a_{11}-s+\sum_{i=1}^{m} b_{i, 11} e^{-s \xi_{i}} & a_{12}+\sum_{i=1}^{m} b_{i, 12} e^{-s \xi_{i}} & \ldots & a_{1 n}+\sum_{i=1}^{m} b_{i, 1 n} e^{-s \xi_{i}} \\ a_{21}+\sum_{i=1}^{m} b_{i, 21} e^{-s \xi_{i}} & a_{22}-s+\sum_{i=1}^{m} b_{i, 22} e^{-s \xi_{i}} & \ldots & a_{2 n}+\sum_{i=1}^{m} b_{i, 2 n} e^{-s \xi_{i}} \\ \cdot & \cdot & \cdot \\ \cdot & \cdot & \ddots \\ a_{n 1}+\sum_{i=1}^{m} b_{i, n 1} e^{-s \xi_{i}} & a_{n 2}+\sum_{i=1}^{m} b_{i, n 2} e^{-s \xi_{i}} & \cdots & a_{n n}-s+\sum_{i=1}^{m} b_{i, n n} e^{-s \xi_{i}}\end{array}\right|$.

Evaluating the determinant gives 


$$
M(s)=Q(s)+\sum_{k=1}^{p} R_{k}(s) e^{-s \tau} k
$$

where $Q(s)$ and $R_{k}(s)$ represent ordinary polynomials of order less than or equal to $n$.

$$
\begin{aligned}
& Q(s)=q_{n} s^{n}+q_{n-1} s^{n-1}+\ldots+q_{1} s+q_{0} \\
& R_{k}(s)=r_{k n} s^{n}+r_{k(n-1)} s^{n-1}+\ldots+r_{k 1} s+r_{k 0}
\end{aligned}
$$

The $\tau_{k}$ represent various sums of the time delays resulting from multiplications of the exponentials of the determinant. In this work, we will always multiply the characteristic equation by -1 if this is necessary to make $q_{n}>0$.

To clarify the notation used in Eq. (2-9), consider the following example.

$$
\begin{aligned}
& \dot{x}_{1}(t)=\dot{a}_{11} x_{1}(t)+a_{12} x_{2}(t)+a_{13} x_{3}(t)+x_{1}\left(t-\xi_{1}\right) \\
& \dot{x}_{2}(t)=x_{3}\left(t-\xi_{2}\right) \\
& \dot{x}_{3}(t)=a_{31} x_{1}(t)+a_{32} x_{2}(t)+a_{33} x_{3}(t)+b_{2,32} x_{2}\left(t-\xi_{2}\right)
\end{aligned}
$$

Then

$$
A=\left|\begin{array}{lll}
a_{11} & a_{12} & a_{13} \\
0 & 0 & 0 \\
a_{31} & a_{32} & a_{33}
\end{array}\right| B_{1}=\left|\begin{array}{ccc}
1 & 0 & 0 \\
0 & 0 & 0 \\
0 & 0 & 0
\end{array}\right| \quad B_{2}=\left|\begin{array}{ccc}
0 & 0 & 0 \\
0 & 0 & 1 \\
0 & b_{2,32} & 0
\end{array}\right| .
$$


Therefore,

$$
\begin{array}{ll}
Q(s)=s^{3}-\left(a_{11}+a_{33}\right) s^{2}+\left(a_{11} a_{33}-a_{13} a_{31}\right) s \\
R_{1}(s)=-s^{2}+a_{33} s & \tau_{1}=\xi_{1} \\
R_{2}(s)=-a_{32} s+a_{32} a_{11}-a_{12} a_{31} & \tau_{2}=\xi_{2} \\
R_{3}(s)=a_{32} & \tau_{3}=\xi_{1}+\xi_{2} \\
R_{4}(s)=-b_{2,32} s+a_{11} b_{2,32} & \tau_{4}=2 \xi_{2} \\
R_{5}(s)=b_{2,32} & \tau_{5}=\xi_{1}+2 \xi_{2} .
\end{array}
$$

Often, not every combination of $\xi_{i}$ will be present in the characteristic equation. Since the linear stability is dependent only on the $\tau_{k}$ and not individually on the $\xi_{i}$, we will also refer to the $\tau_{k}$ as the time delays of the system.

$$
\text { often, systems contain only one time delay } \xi \text { occurring in }
$$

several terms. In this case, the characteristic equation takes the form

$$
M(s)=Q(s)+\sum_{k=1}^{p} R_{k}(s) e^{-k s \xi} .
$$

In many instances, the characteristic exponential polynomial may be written

$$
M(s)=Q(s)+R(s) e^{-s \tau}
$$

where $\tau$ will represent a particular sum of the $\xi_{1}$. It is with this equation that we will be primarily concerned in this work. 
Approximation of the Exponential in the Characteristic Equation

One class of approximations which is used with time delay

systems consists of various approximations to the exponential terms in the characteristic equation. Doing this converts the equation again to a polynomial or rational fractional function (Weaver 1963, p. 4) which can then be studied by the conventional procedures. A number of such techniques are discussed below.

Since the exponential function can be expressed by the limit (Truxa1 1955, p. 547),

$$
e^{-\tau s}=\lim _{n \rightarrow \infty}\left[\frac{1}{(1-\tau s / n)}\right]^{n}
$$

one approximation is to substitute this expression with a finite $n$. A similar approach is to approximate the exponential function by the first few terms of the Maclaurin series (Popov 1962, p. 420; Truxal 1955, p. 547). The series for either the positive or negative exponential may be used to give either

$$
e^{-\tau s}=1-\tau s+(\tau s)^{2} / 2 !-(\tau s)^{3} / 3 !+\ldots
$$

or

$$
e^{-\tau s}=\frac{1}{1+\tau s+(\tau s)^{2} / 2 !+(\tau s)^{3} / 3 !+\ldots} \text {. }
$$

clearly, the approximations are valid only for very small values of

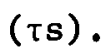

Another of this type is the more sophisticated Pade approximation (Truxal 1955, p. 548). This is a rational algebraic function with numerator polynomial of degree $n$ and denominator polynomial of 
degree $m$, such that a maximum number of terms in the Maclaurin expansion of the approximating function (obtained by long division) agree with similar terms in the expansion of the exponential function. Another approach which uses the Pade approximation is the simulation of the phase function of the time delay frequency response (Truxal 1955, p. 548). Since the time delay has a transfer function whose magnitude is unity at all frequencies, and whose phase angle response is $-\omega \tau$, the approach begins by selecting a rational transfer function with a magnitude of unity and using the Pade approximation to simulate the phase response.

All methods of this class which seek to approximate the exponential terms in the characteristic equation suffer from the same disadvantages. These are 1) the required accuracy of the approximation can only be determined after the approximate expression has been used in the analysis, and 2) the approximations do not necessarily lead to conservative stability results. To exemplify this second disadvantage, Murray and Weaver (1966, p. 45) plotted the stability zones in the parameter space of a simple system obtained by an exact analysis and by an analysis based on the Pade approximation. The approximate analysis gave a significantly larger stability zone than was the actual case, thus leading to the erroneous prediction of stability in regions where in fact the system was unstable. Clearly, this represents a hazardous situation. Although they considered only the Pade approximation for their example, their results are typical for all the approximations of this class. 
Methods of Pontryagin

Several algebraic stability criteria for determining the stability of linear systems with delay were developed by L. S. Pontryagin. Although practical applications remain complicated (Popov.1962, p. 420), these theorems, directed to determining the sign of the real parts of roots of the exponential polynomial, are summarized along with related theorems by'Bellman and Cooke (1963, p. $440 \mathrm{ff.}$ ). Several of these results will be discussed below.

One result which has application to practical system models is based on the notion of a principal term. If $M(y, w)$ is a polynomial in two variables

$$
M(y, w)=\sum_{m, n} a_{m, n} y^{y^{m}} \quad \quad m, n \text { non-negative }
$$

then we call $a_{r t} y^{r}{ }^{t}$ the principal term of the polynomial if $a_{r t} \neq 0$. and if, for each other term $a_{m n} y^{m} w^{n}$ with $a_{m n} \neq 0$, we have either 1) $r>m, t>n, 2) r=m, t>n$, or 3) $r>m, t=n$.

In applications to delayed systems, we will be interested in exponential polynomials of the form $M\left(z, e^{z}\right)$ which would result from a system which has one constant delay. A useful result is given in the following theorem: if the polynomial $\mathrm{M}(\mathrm{y}, \mathrm{w})$ has no principal term, the function $\mathrm{M}\left(\mathrm{z}, \mathrm{e}^{\mathrm{z}}\right)$ has an unbounded number of zeros with arbitrarily Zarge positive real parts. An application of this theorem will be made in the discussion of the Nyquist criterion.

The main result of Pontryagin is given in the following theorem (Bellman and Cooke 1963, p. 441; Pontryagin 1955). Let $M(z)=M\left(z, e^{z}\right)$ be a polynomial with a principal term and let $M(j \omega)=F(\omega)+j G(\omega)$. 
Further, if $\mathrm{v}(\mathrm{a}, \mathrm{b})$ denotes' the change in $\mathrm{M}(\mathrm{j} \omega)$ when $w$ traverses the interval $a<\omega \leqslant b$, call $d v(0, \omega) / d \omega$ the velocity of rotation of $M(j \omega)$. Then if all the zeros of the polynomial lie to the left of the imaginary axis thus implying stability, the vector $M(j \omega)$ for real $\omega$ ranging from $-\infty$ to $+\infty$ continually revolves in the positive direction with a positive velocity. If we let primes denote the derivatives, this criterion can be expressed analytically by the condition

$$
G^{\prime}(\omega) F(\omega)-F^{\prime}(\omega) G(\omega)>0
$$

for. all w.

As indicated previously, the analytic criterion is very difficult to apply to systems of practical size. However, the geometric notion could possibly find application in a computer technique, since it is very similar to the Mikhailov criterion (see the discussion of the Mikhailov criterion).

The Nyquist Criterion

The Nyquist criterion can be extended to study the stability of systems with time delay (Weaver 1963, p. 74; El'sgol'ts 1964, p. 188, 1966, p. 50; Chezem and Seale 1964). To indicate this procedure, we will consider a general $\mathrm{n}^{\text {th }}$ order system with one constant delay, $\xi$, and then give a simple example.

The characteristic equation of this system is

$$
M(s)=Q(s)+\sum_{k=1}^{p} R_{k}(s) e^{-k \xi s}=0 \text {. }
$$

If we apply Pontryagin's theorem on principal terms to this equation, it is evident that for stability to be possible the order of $Q(s)$ must be 
greater than or equal to the largest order of the $R_{k}(s)$. More will be said about this in Chapter 3.

$$
\begin{aligned}
& \text { Dividing } M(s) \text { by } Q(s) \text { gives } \\
& W(s)=M(s) / Q(s)=1+1 / Q(s) \sum_{k=1}^{P} R_{k}(s) e^{-k \xi s} \text {. }
\end{aligned}
$$

If we assume that $Q(s)$ has no purely imaginary zeros, and if we map a T-contour (see Fig. 2.1) from the complex plane of $\mathrm{s}$ to the complex plane of $W(s)-1$, then stability requires that the number of clockwise encirclements of the point $(-1,0)$ in the plane of $W(s)-1$ must equal the number of poles of $W$. in the right half $s$-plane. If $Q(s)$ does have roots on the imaginary axis of the s-plane, then similar results can be obtained by a slight alteration of the $\Gamma$-contour.

To demonstrate the use of this criterion, consider the following characteristic equation:

$$
M(s)=s^{2}+2 s-1+4 e^{-s \tau} \text {. }
$$

Then

$$
W(s)-1=4 e^{-s \tau} /\left(s^{2}+2 s-1\right)
$$

Figure 2.2 shows the Nyquist plot for this equation for $\tau=0.4$, and Fig. 2.3 shows the same curve for $\tau=0.8$. Since $Q(s)$ has one pole in the right-half plane of $s$, stability requires one clockwise encirclement of the $(-1,0)$ point. Therefore, it is clear from Figs. 2.2 and 2.3 that the system is stable for $\tau=0.4$ but unstable for $\tau=0.8$. 


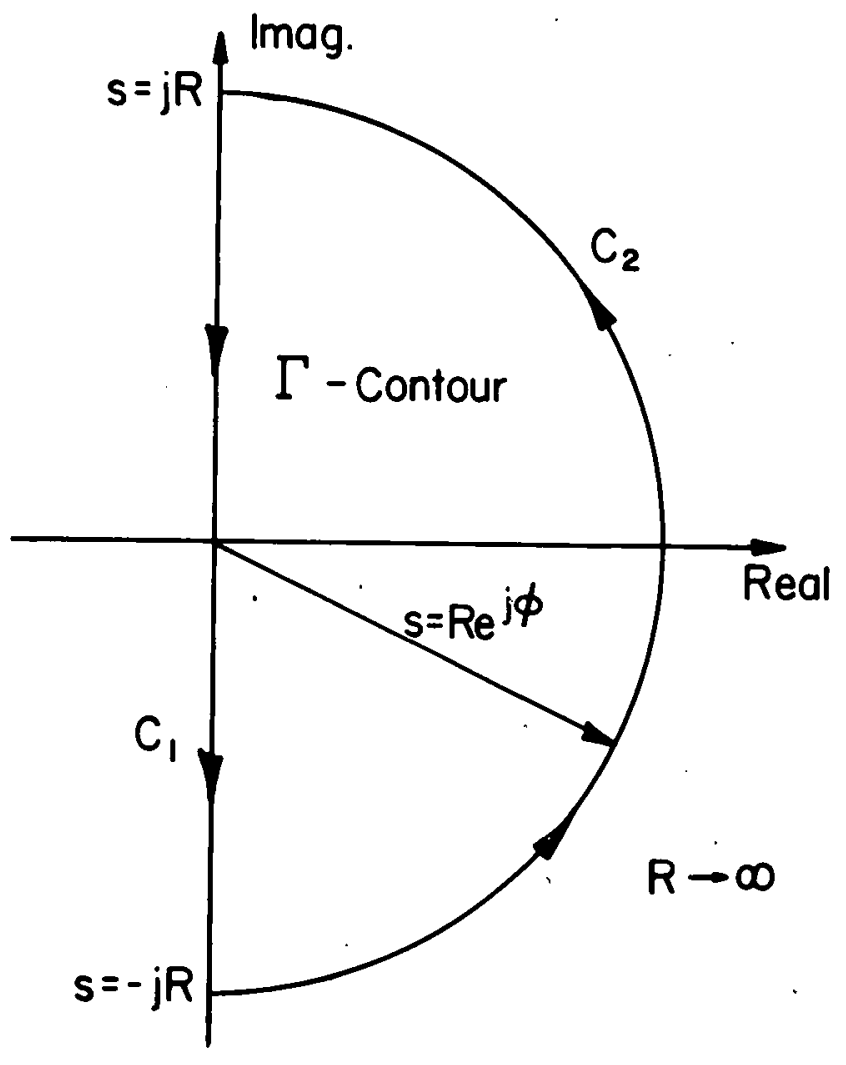

Figure 2.1. $\Gamma$-Contour in S-Plane 


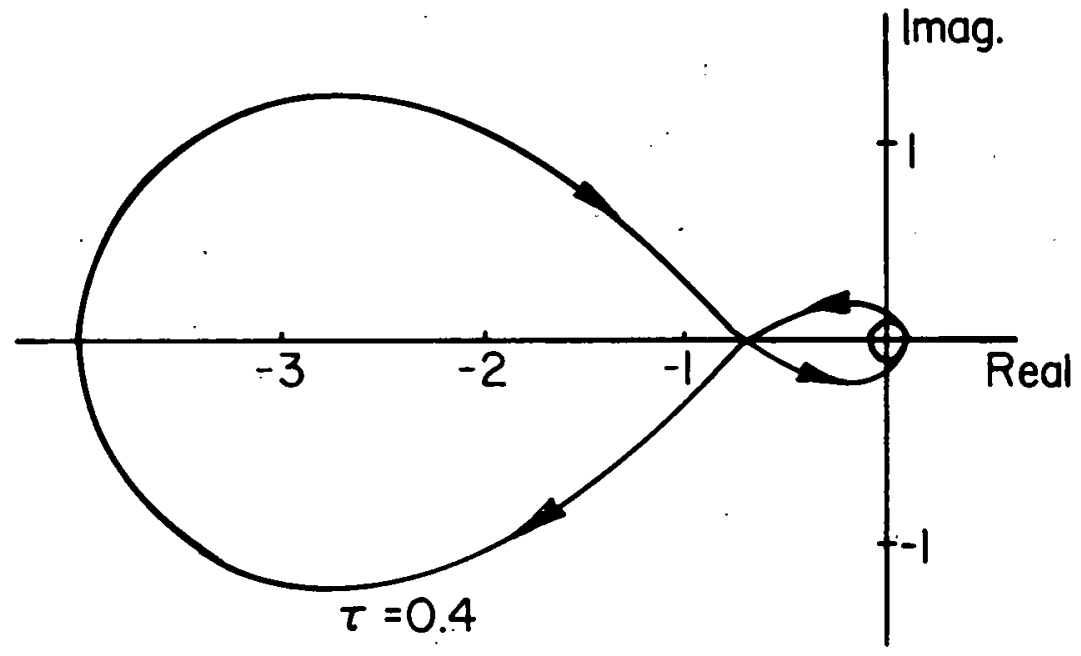

Figure 2.2. Nyquist Diagram. $T=0.4$

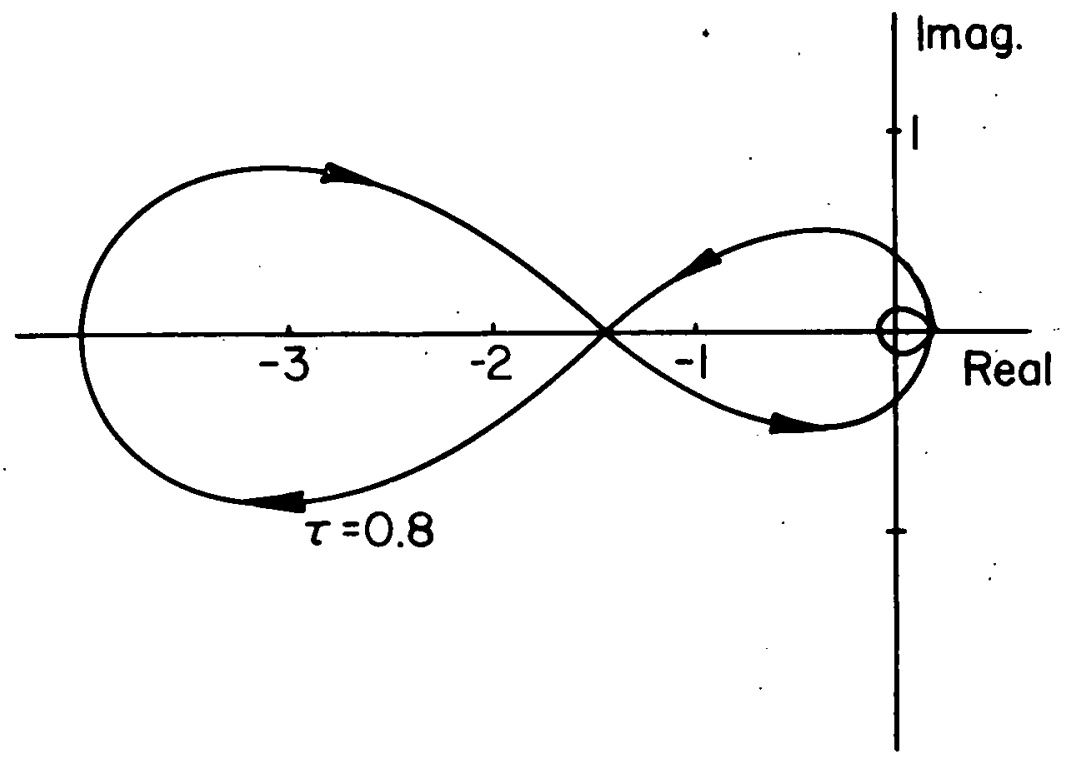

Figure 2.3. Nyquist Diagram. $\tau=0.8$ 
Although this method is not tractable for analytic studies of systems of practical order, it may be possible to develop a computer technique which will increase its applications.

The Mikhailov Critẹion

A method which has been used with some success to study delayed systems is the Mikhailov criterion (Wright and Kerlin 1968; Popov 1962, p. 419 ff.), also called the Leonhard criterion (Lehnigk 1966, p. $152 \mathrm{ff.})$. Like the Nyquist, this is a geometric stability criterion based on the idea of a Mikhailov curve. If $M(s)=0$ is the characteristic equation of a system, then the Mikhailov curve is the image of the positive imaginary axis of $s$ in the complex plane of $M(j \omega)$.

The Mikhailov theorem is stated as follows. In order for an $n^{\text {th }}$ order Zinear system to be stable, the vector $\mathrm{M}(j \omega)$ must complete a net rotation of $\mathrm{n} \pi / 2$ as $w$ ranges from zero to infinity. For systems without delay, this implies that for stability the Mikhailov curve must pass through $\mathrm{n}$ quadrants in succession circling the origin of coordinates in a counterclockwise manner.

For a characteristic polynomial of a non-delayed system, stability requires the curve to begin $(\omega=0)$ on the positive real axis of $M(j \omega)$. This is obviously true since it is a necessary condition for stability that all the coefficients of the characteristic polynomial be positive (Popov 1962, p. 242). Also, for delayed systems, the curve must begin on the positive real axis. This will be shown in Chapter 3 . To exemplify the use of this criterion, consider again the characteristic equation 


$$
M(s)=s^{2}+2 s-1+4 e^{-s \tau} \text {. }
$$

As with the Nyquist, we will plot the Mikhailov curve for two different values of $\tau$. If we let

$$
M(j \omega)=X(\omega)+j Y(\omega),
$$

then

$$
\begin{aligned}
& X(\omega)=-\omega^{2}-1+4 \cos (\omega \tau) \\
& Y(\omega)=2 \omega-4 \sin (\omega \tau) .
\end{aligned}
$$

Table 2.1 gives the values of $X$ and $Y$ versus $\omega$ for $\tau=0.4$ and for $\tau=0.8$.

Table 2.1. $X(\omega)$ and $Y(\omega)$ for Mikhailov Curves

\begin{tabular}{ccccc}
$\omega$ & \multicolumn{2}{c}{$\mathrm{X}(\omega)^{\tau}=0.8$} & $\mathrm{Y}(\omega)$ & $\mathrm{X}(\omega)^{\tau}=0.4$ \\
0 & 3.00 & 0.00 & 3.00 & 0.00 \\
0.4 & 2.64 & -0.46 & 2.79 & 0.16 \\
0.8 & 1.57 & -0.79 & 2.16 & 0.34 \\
1.2 & -1.46 & -0.88 & 1.11 & 0.55 \\
1.6 & -2.41 & -0.63 & -0.35 & 0.81 \\
2.0 & -5.12 & 0.00 & -2.21 & 1.13 \\
3.0 & -13.0 & 3.30 & -8.55 & 2.27 \\
4.0 & -21.0 & 8.23 & 117 & 4.00 \\
10.0 & -102 & 16.0 & -104 & 23.0 \\
$\infty$ & $-\infty$ & $\infty$ & $-\infty$ & $\infty$
\end{tabular}


The two Mikhailov curves are shown in Fig. 2.4. Since the order of this system is two, stability requires the Mikhailov curve to pass through a net angle of $\pi$ as $\omega$ proceeds to infinity. Clearly, from Fig. 2.4, the example system is stable for a time delay 0.4 but unstable for a delay of 0.8 .

It is evident from the above exercise that a computer technique would be necessary to utilize the Mikhailov method for systems of practical size. Some of the difficulties encountered with this approach will be discussed in the next section.

The Modified Mikhailov Criterion

In numerical applications of the Mikhailov criterion, the major shortcoming arising from the study of large systems is the necessity of obtaining a large enough number of points to place the Mikhailov curve in each successive quadrant. This problem is amplified by the ignorance of the values of $\omega$ at which the points should be calculated. These difficulties pervade the Nyquist and Pontryagin methods as well.

In an effort to overcome these shortcomings, Wright and Kerlin (1968, 1969) have modified the Mikhailov criterion to state that the Zinear system is stable if and only if the Zocus of $L(j \omega)$ for $-\infty \leqslant \omega \leqslant+\infty$ does not have any net clockwise encirclements of the origin. L(jw) is given by

$$
L(j \omega)=\frac{M(j \omega)}{\prod_{i=1}^{n}\left(j \omega+\left|a_{i i}\right|\right)}
$$

where $a_{i i}$ represents the diagonal elements of $A$, and $M(j \omega)$ is the characteristic equation of the system. Wright and Kerlin use a very 


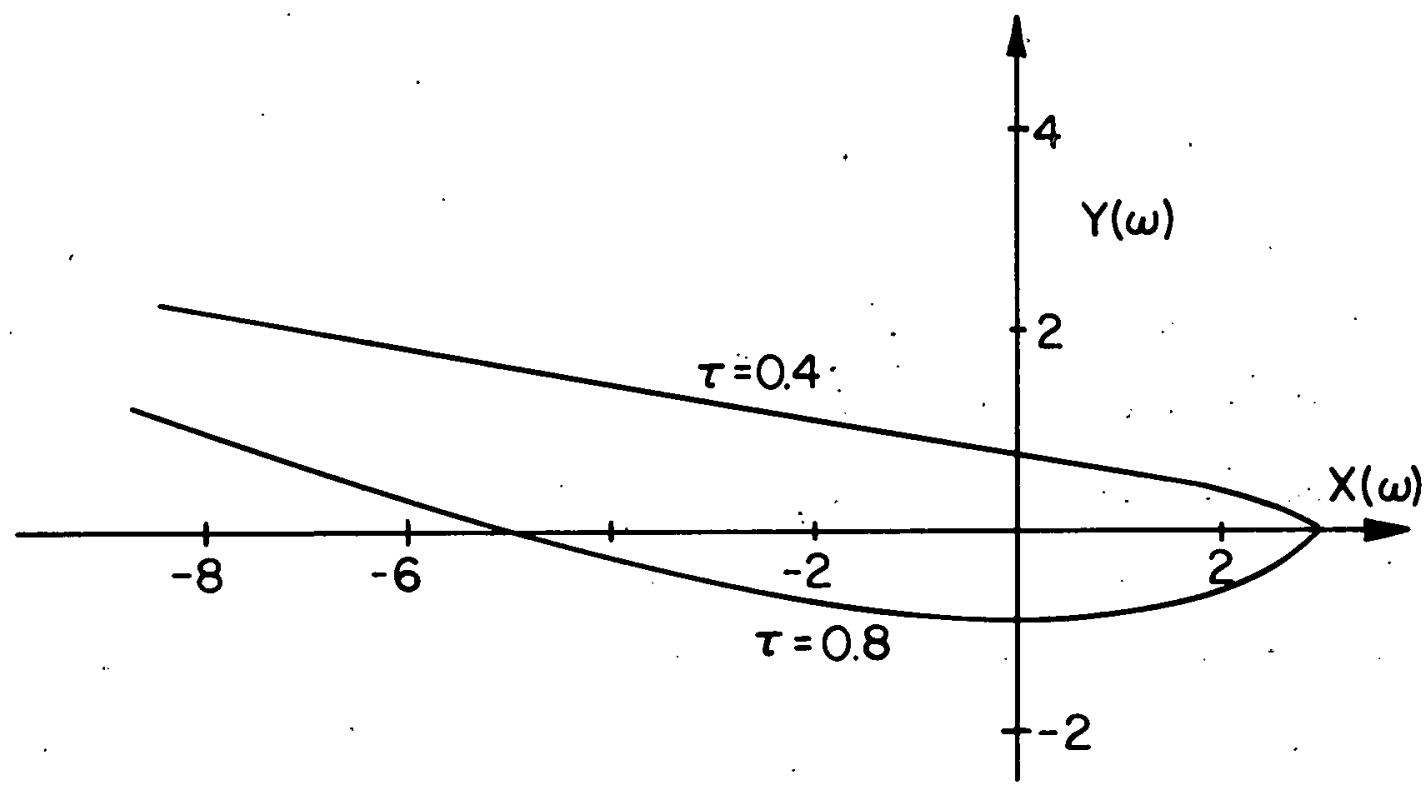

Figure 2.4. Mikhailov Curves 
efficient algorithm for calculating the determinants of $L(j \omega)$ for each value of $w$. The technique has been used successfully for systems of order over 100.

To demonstrate the use of this criterion, consider again the characteristic equation given earlier.

$$
M(s)=s^{2}+2 s-1+4 e^{-s \tau}
$$

Figure 2.5 shows the modified Mikhailov curves for $\tau_{1}=0.4$ and $\tau_{2}=0.8$. As expected, the stability results are the same as given by the Mikhailov criterion. Figure 2.5 shows that the modified plots approach the point $(1,0)$ as a limit rather than infinity. This offers a definite advantage in the computer applications to large systems where otherwise scaling might be necessary. The modification may be thought of as a kind of normalization of both magnitude and phase. (This technique is similar to dividing the characteristic equation by $Q(s)$ when employing the Nyquist criterion.)

\section{Nonlinear Methods}

In earlier works (e.g. Brehm, et. al. 1968; Devooght and Smets 1967), Liapunov's direct method has been used to study the stability of nonlinear nuclear systems; and Brehm, et. al. (1968) compared several different systematic approaches of constructing Liapunov functions for nuclear systems. In this section the extension of Liapunov's theorems to systems with delayed arguments will be discussed.

Briefly, the concept of Liapunov's direct method is as follows. If a V-function can be constructed in some domain of the state variables 


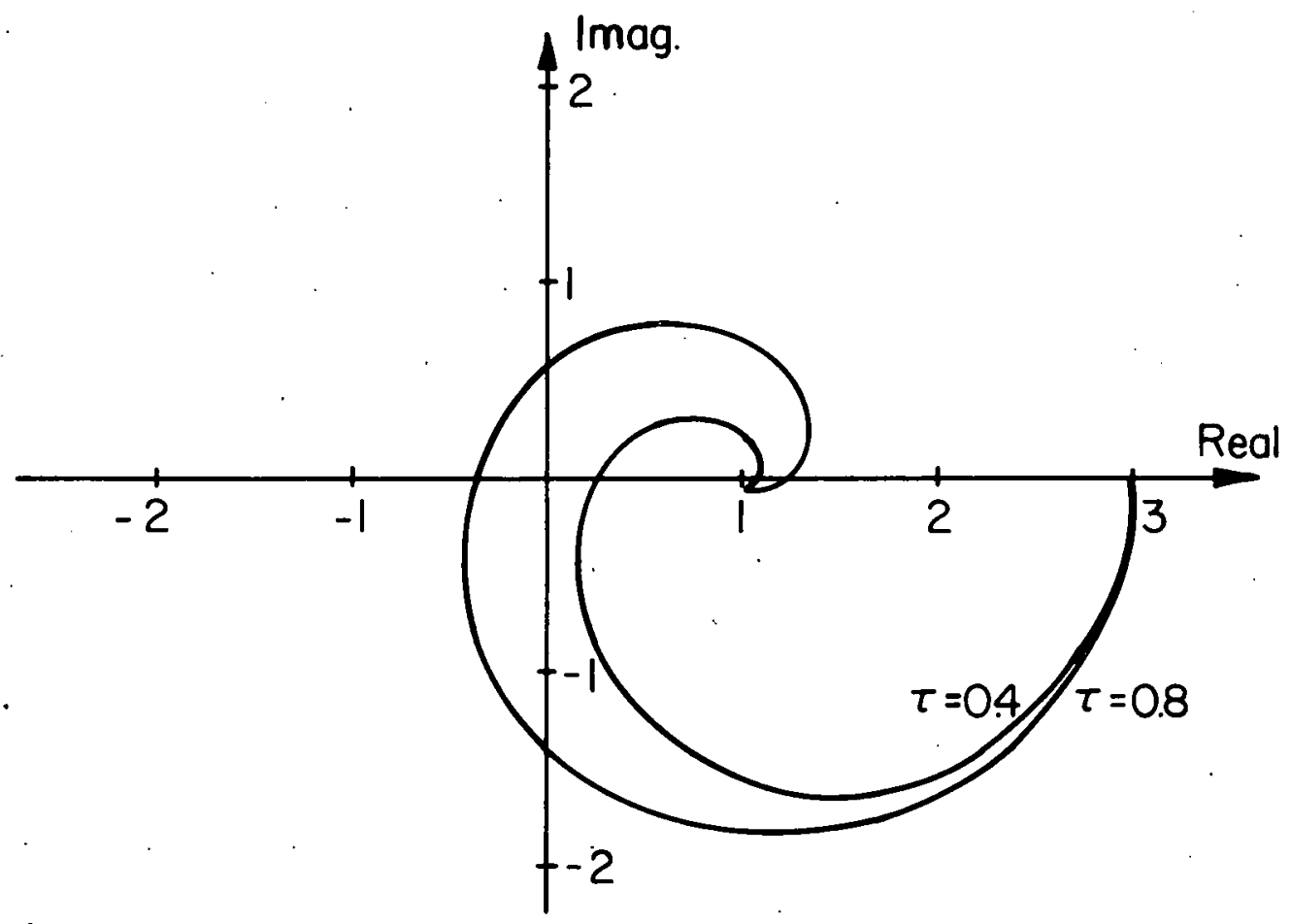

Figure 2.5. Modified Mikhailov Diagrams 
$\vec{x}$ in which the following four properties are satisfied,

1) $V(\vec{x})$ and its first partial derivatives are continuous,

2) $V(\overrightarrow{0})=0$,

3) $V(\vec{x})>0 \quad \vec{x} \neq \overrightarrow{0}$, and

4) $\dot{V}(\vec{x})<0$ (the dot denotes the total time derivative),

then the system is asymptotically stable within that domain. Since the conditions of the theorem are only sufficient for stability, the construction of the V-function becomes the fundamental problem in applying the method.

The theorems of Liapunov can be applied directly to systems with time delay (El'sgol'ts 1966, p. 64; 1964, p. 192), with the inclusion of delay implying the time derivative of the Liapunov function will include the delayed terms. Nevertheless, if the derivative can be shown to be negative definite, the stability results are the same. Unfortunately, this approach to nonlinear stability is specious, being applicable to only a few contrived examples (El'sgol'ts 1966;1964).

The theorems of Liapunov's direct method have been generalized in order to be more useful for studying time delayed systems (Krasovskii 1963, p. 126; Driver 1962), but these generalizations have been used only with limited success (Razumikhin 1960; Murray and Weaver 1966). They do not lead to changes of the fundamental concepts of the second method; the idea is still to find a positive-definite Liapunov function and to draw stability conclusions from the negative-definiteness of its time derivative. Nevertheless, certain modifications are necessary. Driver (1962) states and proves these more general theorems. They take 
into account the fact that with delayed systems the Liapunov function may exhibit an oscillatory nature (alternately positive and negative time derivative), rather than a strict monotonically decreasing nature (negative time derivative), and still represent a stable system. Hence, the stability results have been generalized to include time derivatives which are alternately positive and negative.

Murray and Weaver (1966) applied Liapunov's direct method to a delayed model describing coupled-core reactor systems. In this study quadratic $V$-functions of the form

$$
v(\vec{x})=\sum_{i=1}^{n} x_{i}^{2}
$$

were employed. They discovered that this method quickly lost its tractability as the order of the system increased.

Others have suggested different forms of the $V$-function. Krasovskii (1963, p. 170) suggests the use of the form

$$
v(\vec{x})=v(\vec{x})+\sum_{j=1}^{n} \int_{t-\tau}^{\tau} x_{j}^{2}(u) d u
$$

where $v(\vec{x})$ is a quadratic form developed from the system equations with delayed terms set to zero. Although this expression possesses certain advantages for special problems, it is not generally useful (Murray and Weaver 1966, p. 46). Razumikhin (1960) has suggested a substitution which provides stability results as a function of $\tau$ by using a meanvalue approach to the delayed terms.

Although Liapunov's direct method is powerful in its range of applicablity to both delayed and regular systems, it is not practical 
to apply the method in analytic form to any but very low order systems. Some work has been done in the area of numerical applications of the direct method (Margolis and Vogt 1963; Rodden 1964), but to this author's knowledge none have been extended to include time delays or even applied to practical systems without delay. Liapunov's direct method does not appear promising as a technique for studying the stability of systems of practical order.

The well-known Poincaré-Liapunov theorem, which states that if a system is linearly stable then it is also nonlinearly stable in a sufficiently small region of the equilibrium, has direct extensions to models with time delay (Bellman and Cooke 1963, p. 335). Use of this knowledge may lead to a practicable method for studying delayed, nonlinear system stability based on the linear stability results.

\section{Summary of Existing Methods}

In the discussion of methods normally applied to delayed systems, we have seen that 1 ) approximations to the time delay terms while simplifying the analysis may lead to nonconservative stability predictions, 2) the nonlinear methods of Liapunov are very difficult to apply to practical models, and 3) linear methods vary in their ease of application. In the remaining portion of this work, we will confine our interest to linear systems. We have seen from the Poincare-Liapunov theorem that linear stability implies nonlinear stability in at least a restricted domain. Further, linear stability is currently a requirement in the design of nuclear systems. 
of the several linear methods discussed in this chapter, the Mikhailov appears most appropriate for a parametric stability analysis because the least work is required. Although the same information can be obtained by any of the. Pontryagin, Nyquist, Mikhailov, and Modified Mikhailov results, only the Mikhailov operates directly from the characteristic equation.

In the determination of absolute stability (i.e., stability for a completely specified set of parameters), the computational advantages of the Modified Mikhailov over the Mikhailov have already been discussed. In addition the Modified Mikhailov criterion is simpler to use than the Nyquist since the roots of a polynomial need not be found, and simpler than the Pontryagin result because derivatives are unnecessary. In the following chapters, we will develop and exhibit a technique of performing a parametric stability analysis based on the Mikhailov and Modified Mikhailov stability criterions. 


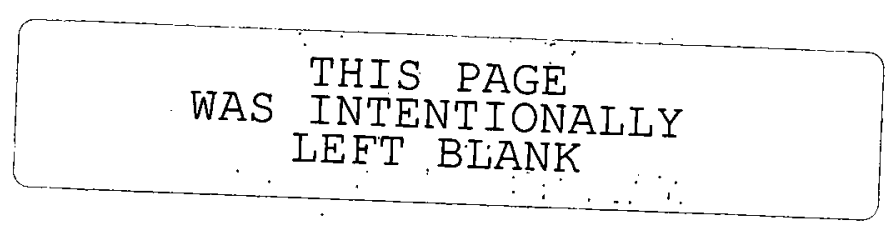




\section{CHAPTER 3}

\section{PARAMETRIC STABILITY ANALYSIS \\ OF SYSTEMS WITH TIME DELAY}

In this chapter, a technique of analyzing the parametric stability of delayed systems will be developed. The method is based on the Mikhailov stability criterion discussed earlier. Since a proof of the Mikhailov criterion for delayed systems has apparently not appeared in English, a proof will be given here. Later, methods of mapping stability regions in the parameter space which are conditional on the value of the time delay will be developed through utilization of Déscartes' rule of signs and Sturm's theorem.

\section{The Mikhailov Criterion for Delayed Systems}

Before proving the Mikhailov criterion, we will first prove the logarithmic derivative theorem on which the Mikhailov criterion is based.

\section{Logarithmic Derivative Theorem. (Churchill 1960, p. 261):}

Let a single valued function $\mathrm{f}$ be analytic inside and on a closed contour, $\mathrm{C}$; and let $\mathrm{f}$ have no zeros on $\mathrm{C}$ and at most a finite number of zeros interion to $\mathrm{C}$. Then if $\mathrm{C}$ is described in the positive (counterclockwise) sense,

$$
\frac{1}{2 \pi j} \int_{C} \frac{f^{\prime}(z)}{f(z)} d z=N_{0}
$$


where $\mathrm{N}_{0}$ is the total number of zeros inside $\mathrm{c}$, a zero of order $\mathrm{m}$ being counted m times.

Proof: Let $D$ be the region enclosed by $C$. Since there are only a finite number of zeros in $D$, and since zeros of analytic functions are isolated (Churchill 1960, p. 150), we may write

$$
f(z)=\left\{\prod_{i=1}^{n}\left(z-z_{i}\right)^{m_{i}} g(z) \quad g(z) \neq 0 \text { if } z \in D \cup C\right. \text {. }
$$

where the $z_{i}$ represent the $n$ distinct zeros of $f$ each with an order $m_{i}$. Therefore,

$$
f^{\prime}(z)=\left\{\prod_{i=1}^{n}\left(z-z_{i}\right)^{m_{i}}\right\} g^{\prime}(z)+\left[\sum_{k=1}^{n} \frac{\prod_{i=1}^{n}\left(z-z_{i}\right)^{m_{i}}}{\left(z-z_{k}\right)^{m_{k}}}\left(z-z_{k}\right)^{m_{k}-1_{m_{k}}}\right] g(z) \text {. }
$$

Then

$$
f^{\prime}(z) / f .(z)=\frac{\prod_{i=1}^{n}\left(z-z_{i}\right)^{m_{i}}\left[g^{\prime}(z)+g(z) \sum_{k=1}^{n} \frac{m_{k}}{z-z_{k}}\right]}{\prod_{i=1}^{n}\left(z-z_{i}\right)^{m_{i}}\{g(z)\}}
$$

Or

$$
f^{\prime}(z) / f(z)=g^{\prime}(z) / g(z)+\sum_{k=1}^{n} \frac{m_{k}}{z-z_{k}} \text {. }
$$

Now since $g^{\prime}(z) / g(z)$ is analytic inside and on $C^{\prime}$, Cauchy's residue theorem (Churchill 1960, p. 155) gives

$$
\begin{aligned}
\frac{1}{2 \pi j} \int_{C} \frac{f^{\prime}(z)}{f(z)} d z & =\frac{1}{2 \pi j} \int_{C} \frac{g^{\prime}(z)}{g(z)} d z+\frac{1}{2 \pi j} \sum_{k=1}^{n} \int_{C} \frac{m_{k}}{z-z_{k}} d z \\
& =0+\sum_{k=1}^{n} m_{k} \\
& =N_{0} \quad \text { Q.E.D. }
\end{aligned}
$$


From the above theorem, the argument principle (Churchill 1960, p. 272; El'sgol'ts 1966, p. 50) may be developed. Since

$$
d / d z[\log f(z)]=f^{\prime}(z) / f(z),
$$

the function $\log (f)$ is the indefinite integral of $\mathrm{f}^{\prime} / \mathrm{f}$. Consider a general closed contour $C$ for which $f(z)$ satisfies the conditions of the above theorem, and 1 et $B_{1}$ and $B_{2}$ represent the same point at the beginning and end of the contour, respectively, as shown in Fig. 3.1. In addition if we let

$$
\begin{aligned}
& f\left(B_{1}\right)=A_{1} e^{j \theta_{1}} \\
& f\left(B_{2}\right)=A_{2} e^{j \theta},
\end{aligned}
$$

we have

$$
\begin{aligned}
\int_{C} d[\log f(z)] & =\int_{B}^{B}{ }^{B} d[\log f(z)]=\log \left[f\left(B_{2}\right) / f\left(B_{1}\right)\right] \\
& =\log \left[\frac{A_{2} e^{j \theta_{2}}}{A_{1} e^{j \theta_{1}}}\right]=2 \pi j N_{0} .
\end{aligned}
$$

And since $\mathrm{A}_{2}=\mathrm{A}_{1}$, we have

$$
\begin{aligned}
& j\left(\theta_{2}-\theta_{1}\right)=2 \pi j N_{0} \\
& \left(\theta_{2}-\theta_{1}\right)=2 \pi N_{0} .
\end{aligned}
$$

We have therefore proved the following theorem.

Argument Principle: Given a closed contour $c$ for which $\mathrm{f}(\mathrm{z})$ satisfies the conditions of the Zogarithmic derivative theorem, then the argument of $\mathrm{f}(\mathrm{z})$ will increase by $2 \pi \mathrm{N}$ radians as $z$ completes one positive revolution about $c$. 


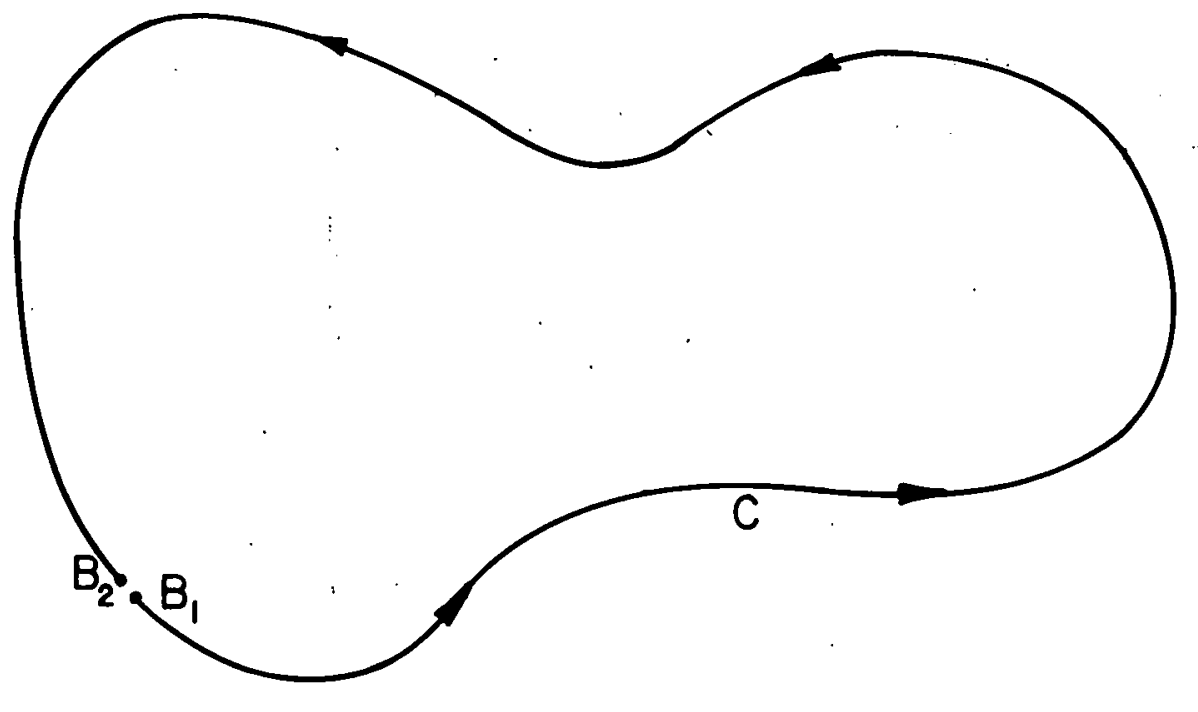

Figure 3.1. Closed Contour C 
When the logarithmic derivative theorem or the argument principle is applied to stability. problems through the Nyquist or similar stability criterions, it is generally tacitly assumed that when the closed contour is extended to cover the entire right-half plane, the function $f$ still has only a finite number of zeros within $C$. Usually this assumption is justified since the theorems are primarily used to analyze polynomials or rational algebraic functions which indeed have only a finite number of zeros in the whole space. However, for the transcendental exponential polynomials with their infinite number of zeros, other considerations are necessary.

By using the Bolzano-Weierstrass theorem (Buck 1956, p. 10), which states that every bounded, infinite set has at least one cluster point, it is possible to show that if $f$ is analytic on a closed bounded region, then there are only a finite number of zeros of $f$ in the region (DePree and Oehring 1969; pp. 177, 199). Hence, in the statements of the logarithmic derivative theorem and the argument principle, we may drop the assumption of the finite number of zeros of $f$ if we require the contour to be bounded as well as closed.

However, in extending the contour to cover the entire righthalf plane, we no longer have a bounded region; and some other considerations must be used to assure there are only a finite number of zeros within the contour.

Pinney (1958, p. $41 \mathrm{ff.}$ ) and Pontryagin (1955) have considered this problem. Pontryagin (see Chapter 2) has shown that if the characteristic exponential polynomial does not have a principal term there are an infinite number of roots in the right-half plane. Pinney shows that, 
in addition to a finite number of roots in any neighborhood of the origin, exponential polynomials will have chains of more-or-less evenly spaced roots extending to infinity.

Consider the general exponential polynomial of the form given in $\mathrm{Eq} \cdot(2-9)$,

$$
M(s)=Q(s)+\sum_{k=1}^{p} R_{k}(s) e^{-s \tau_{k}}
$$

and let $\mu$ be the order of the $Q(s)$ polynomial and $\nu$ be the largest order of the $R_{k}(s)$ polynomials. Then in order for $M(s)$ to have a principal term, we must have $\mu \geqslant \nu$. Pinney (1958) shows, if $\mu>\nu$, there can only be a finite number of roots with real parts greater than any particular value. Asymptotic values will have real parts approaching negative infinity. If. $\mu=\nu$, it is possible that the exponential polynomial will have chains of asymptotic roots whose real parts approach constants asymptotically. Whether or not any of these constants lie to the right of the imaginary s-axis will determine whether or not there will be a finite number of roots with positive real parts. If $\mu<v$, then the real parts of the asymptotic roots will increase indefinitely as the Pontryagin results indicated.

Therefore by the above considerations we can assure that the exponential polynomial has only a finite number of roots with positive real parts if we require $\mu>\nu$. We will not consider the stability of systems with $\mu=\nu$ since additional assumptions are required and since this case is physically unimportant. If $\mu>\nu$, we will say McK; and we can apply the argument principle to the $\Gamma$ - contour (see Fig. 2.1) enclosing the entire right-half plane. 
Under the conditions guaranteeing a finite number of roots in the right-half plane, the Mikhailov criterion is a restatement of the argument principle.

Mikhailov Stability Criterion: If $\mathrm{M}(\mathrm{s})=0$ with $\mathrm{M}$ given by Eq. (3-11) represents the characteristic equation of an $n^{\text {th }}$ order inear system of differential equations with constant time delays and $\mathrm{M}(s) \varepsilon \mathrm{K}$, then stability requires the vector $\mathrm{M}(j \omega)$ to complete a net rotation of an $\Delta \theta=\mathrm{n} \pi / 2$ as $\omega$ ranges from zero to infinity.

Proof: In order to prove the theorem, we will apply the argument principle to $M(s)$ on the $\Gamma$-contour shown in Fig. 2.1. Since $M(s)$ $\varepsilon K$, there are only a finite number of roots of $M(s)$ in the right-half plane. We now divide the $\Gamma$-contour into two segments: $C$ consisting of the imaginary axis with $s=j R,-\infty \leqslant R \leqslant \infty$, and $C_{2}$ consisting of the semicircular portion with $s=R e^{j \phi},-\pi / 2 \leqslant \phi \leqslant \pi / 2, R \rightarrow \infty$. On $\mathrm{C}_{2}$, the characteristic equation can be written

$$
\begin{aligned}
M(s)= & q_{n} R^{n}[\operatorname{cosn} \phi+j \operatorname{sinn} \phi]+q_{n-1} R^{n-1}[\cos (n-1) \phi+j \sin (n-1) \phi]+ \\
& \cdots+q_{0}+\sum_{k=1}^{p}\left\{r_{k(n-1)} R^{n-1}[\cos (n-1) \phi+j \sin (n-1) \phi] e^{-R \tau} e^{j \phi}\right. \\
& \left.+\cdots+r_{k_{0}} \exp \left(-R \tau_{k} e^{j \phi}\right)\right\}
\end{aligned}
$$

Now from the summation, we can write a typical term as

$$
\begin{gathered}
r_{k(n-1)} R^{n-1} e^{j(n-1) \phi_{e}} e^{-R \tau_{k} e^{j \phi}}=r_{k(n-1)} R^{n-1} e^{j(n-1) \phi} \operatorname{exp[-R\tau _{k}\operatorname {cos}\phi -jR\tau _{k}\operatorname {sin}\phi ]} \\
\quad=r_{k(n-1)} R^{n-1} e^{-R \tau_{k} \cos \phi} e^{j\left[(n-1) \phi-R \tau_{k} \sin \phi\right]}
\end{gathered}
$$


Therefore,

$$
\begin{aligned}
M(s)= & q_{n} R^{n}[\cos \phi+j \operatorname{sinn} \phi]+q_{n-1} R^{n-1}[\cos (n-1) \phi+j \sin (n-1) \phi] \\
& +\ldots+q_{0}+\sum_{k=1} e^{-R \tau_{k} \cos \phi}\left\{r _ { k ( n - 1 ) } R ^ { n - 1 } \left[\cos \left((n-1) \phi-R \tau_{k} \sin \phi\right)+\right.\right. \\
& \left.j \sin \left((n-1) \phi-R \tau_{k} \sin \phi\right)\right]+r_{k(n-2)} R^{n-2}\left[\cos \left((n-2) \phi-R \tau_{k} \sin \phi\right)\right. \\
& \left.\left.+j \sin \left((n-2) \phi-R \tau_{k} \sin \phi\right)\right]+\ldots+r_{k 0}\left[\cos \left(R \tau_{k} \sin \phi\right)-j \sin \left(R \tau_{k} \sin \phi\right)\right]\right\} .
\end{aligned}
$$

Hence the argument or phase $\theta$ of $M(s)$ on $C_{2}$ may be written

$$
\operatorname{Tan} \theta=\frac{\left[\begin{array}{l}
q_{n} R^{n} \sin (n \phi)+q_{n-1} R^{n-1} \sin (n-1) \phi+\ldots+\sum_{k=1} e^{-R \tau} k^{\cos \phi} x \\
x\left\{r_{k(n-1)} R^{n-1} \sin \left((n-1) \phi-R \tau_{k} \sin \phi\right)-\ldots-r_{k 0} \sin \left(R \tau_{k} \sin \phi\right)\right\}
\end{array}\right]}{\left[\begin{array}{l}
q_{n} R^{n} \cos (n \phi)+q_{n-1} R^{n-1} \cos (n-1) \phi+\ldots+q_{0}+\sum_{k=1} e^{-R \tau_{k} \cos \phi} x \\
x\left\{r_{k(n-1)} R^{n-1} \cos \left((n-1) \phi-R \tau_{k} \sin \phi\right)+\ldots+r_{k_{0}} \cos \left(R \tau_{k} \sin \phi\right)\right\}
\end{array}\right] .}
$$

Dividing the numerator and denominator by $\mathrm{R}^{\mathrm{n}}$ gives

$$
\operatorname{Tan} \theta=\frac{\left[\begin{array}{l}
q_{n} \sin (n \phi)+\left(q_{n-1} / R\right) \sin (n-1) \phi+\ldots+\sum_{k=1}^{p} e^{-R \tau} k^{\cos \phi} x \\
x\left\{\left(r_{k(n-1)} / R\right) \sin \left((n-1) \phi-R \tau_{k} \sin \phi\right)-\ldots-\left(r_{k 0} / R^{n}\right) \sin \left(R \tau_{k} \sin \phi\right)\right\}
\end{array}\right]}{\left[\begin{array}{l}
q_{n} \cos (n \phi)+\left(q_{n-1} / R\right) \sin (n-1) \phi+\ldots+\left(q_{0} / R^{n}\right)+\sum_{k=1}^{p} e^{-R \tau_{k} \cos \phi} x \\
x\left\{\left(r_{k(n-1)} / R\right) \cos \left((n-1) \phi-R \tau_{k} \sin \phi\right)+\ldots+\left(r_{k_{0}} / R^{n}\right) \cos \left(R \tau_{k} \sin \phi\right)\right\}
\end{array}\right] .}
$$

Now since $|\cos \alpha| \leqslant 1$ and $|\sin \alpha| \leqslant 1$ for any $\alpha$, and since on $C_{2}$ $(-\pi / 2 \leqslant \phi \leqslant \pi / 2) \cos \phi \geqslant 0$, we have as $\mathrm{R} \rightarrow \infty$ 


$$
\operatorname{Tan} \theta=\sin (n \phi) / \cos (n \phi)=\operatorname{Tan}(n \phi)
$$

or

$$
\theta=\mathrm{n} \phi+\mathrm{m} \pi \quad \mathrm{m} \text { an integer. }
$$

Therefore, the change in argument of $M(s)$ on $C_{2}$ is given by

$$
\Delta \mathrm{ARG}_{C_{2}}[M(s)]=\mathrm{n} \pi / 2+\mathrm{mm}-(-\mathrm{n} \pi / 2+\mathrm{m} \pi)=\mathrm{n} \pi
$$

Now from the argument principle we can write.

$$
\Delta A R C_{1}[M(s)]+\Delta A R G_{C}[M(s)]=2 \pi N_{0} \text {. }
$$

Therefore,

$$
\Delta A R C_{C_{1}}[M(s)]=2 \pi N_{0}-n \pi \cdot
$$

Now if we reverse the direction of integration along $c_{1}$ and note symmetry about the real axis, we have

$$
\begin{aligned}
\underset{0 \leqslant \omega \leqslant \infty}{\triangle A R G}[M(j \omega)] & =1 / 2\left(n \pi-2 \pi N_{0}\right) . \\
& =n \pi / 2-\pi N_{0}
\end{aligned}
$$

However, for stability $\mathrm{N}_{0}=0$. Hence stability requires

$$
\underset{0 \leqslant \omega \leqslant \infty}{\Delta A R G[M(j \omega)]}=n \pi / 2 \quad \text { Q.E.D. }
$$

In developing Eq. (3-18), we have seen that as the magnitude of $s$ goes to infinity, the argument $M(s)$ is equal to $n$ times the argument of $s$. Therefore, as $s$ goes to positive infinity along the imaginary axis, we have (assuming $\mathrm{m}=0$ )

$$
\theta=\mathrm{n} \pi / 2
$$


Therefore since for stability the argument must increase by $n \pi / 2$ radians as $\omega \rightarrow \infty$, and since as $\omega \rightarrow \infty \theta \rightarrow n \pi / 2$, stability requires the argument to begin $(\omega=0)$ at $\theta=0$. This in turn requires the constant terms of the $Q(s)$ and $R_{k}(s)$ polynomials to sum to a value greater than zero.

From this argument notion of the Mikhailov stability criterion, it is possible to develop a criterion based on the locus of all points traced out by the tip of the vector $M(j \omega)$ as $\omega$ ranges from zero to infinity. This locus is called the Mikhailov curve. Stability requires the Mikhailov curve to pass through a net number of $n$ quadrants in a counterclockwise fashion as $w$ goes from zero to infinity. The curve must begin on the positive real axis.

\section{Critical Time Delays and the Existence Of Regions of Conditional Stability}

For delayed as well as non-delayed linear systems, we know (Bellman and Cooke 1963, p. 115) that the passage of a system from a region of stability to a region of instability is connected with the passage of roots through purely imaginary values or through the origin. In this section, we will show how this fact can be used to calculate critical time delays, and how regions of conditional stability can be found.

The requirement of the existence of purely imaginary roots (including the origin) of the characteristic equation, $M(s)=0$, is that $M(j \omega)=0$. for some value of $\omega$. (Since $M$ is symmetric about the real axis, this condition need only be checked for non-negative values of $\omega_{.}$) However, it is seen that this condition is the same as requiring the 
Mikhailov curve to pass through the origin for some positive $\omega$. Therefore if, by a small change of the coefficients or of the time delays, we can make the Mikhailov curve pass to the opposite side of the origin, we have driven a pair of roots across the imaginary axis. This does not indicate where the remaining roots are located, and so direct stability conclusions. can not be drawn from this alone. We can say that if the system is stable and we cause roots to cross the imaginary axis then the system becomes unstable. We can not say that if we cause a pair of roots of an unstable system to cross the axis, then it becomes stable. This actually could be the case, but not necessarily since we do not know the location of the other roots. We can use these considerations to our advantage in determining how time delays affect system stability. To introduce the technique of determining critical time delays and regions of a conditional stability, we will discuss several relatively simple examples. Later, we will develop in more detail the stability analysis technique.

Following Popov (1962,.pp. $421 \mathrm{ff.})$, consider a characteristic equation giving a Mikhailov curve equation of the form

$$
M(j \omega)=Q(j \omega)+e^{-j \omega \tau}
$$

or

$$
M(j \omega)=M_{1}(\omega) e^{j \phi_{1}(\omega)}+e^{-j \omega \tau}
$$

where $M_{1}(\omega)$ is the modulus and $\phi_{1}(\omega)$ the argument or phase of $Q(j \omega)$. The Mikhailov curve of this system can be obtained by the geometric addition of the auxiliary curve $(Q(j \omega)$ ) and the unit vector rotated by the correct angle. 
Consider the example shown in Fig. 3.2a. The auxiliary system is stable and has a magnitude greater than one for all positive values of w. Therefore, the resultant curve (typically shown in Fig. 3.2b), while taking a distorted pattern, will encircle the origin in the same manner and rotate by the same angle as the auxiliary system. In this case, stability is unaffected by the value of the time delay.

Now consider another example which is depicted in Fig. 3.3. In this case the auxiliary curve is stable but intersects the unit circle once. Because of this intersection, it is possible that the geometric addition of the two vectors will cause the resultant curve to take a different position with respect to the origin thus indicating that a stability boundary has been crossed. We wish to discover how the value of the time delay is related to this boundary.

Since $M_{1}\left(\omega_{k}\right)=1$, the resultant curve will pass through the origin if

$$
\phi_{1}\left(\omega_{k}\right)=-\omega_{k} \tau+\pi+2 \pi q
$$$$
q=0,1,2, \ldots
$$

Hence the critical time delays (those which cause the resultant curves to pass through the origin) are given by

$$
\tau_{k q}=1 / \omega_{k}\left[\pi-\phi_{1}\left(\omega_{k}\right)+2 \pi q\right] \quad q=0,1,2, \ldots .
$$

Now suppose this system is stable for $\tau<\tau_{k_{0}}$. Then it is true that the resultant curve will pass through the origin at $\tau=\tau_{k_{0}}$, and will become unstable for all values of $\tau>\tau_{k_{0}}$. That this is true can $\ddot{b e}$ : seen by considering the resultant curves for values of $\tau$ slightly less than $\tau_{k l}$ (Fig. 3.4a) and slightly greater than $\tau_{k l}$ (Fig. 3.4b). The system is unstable in each case. 


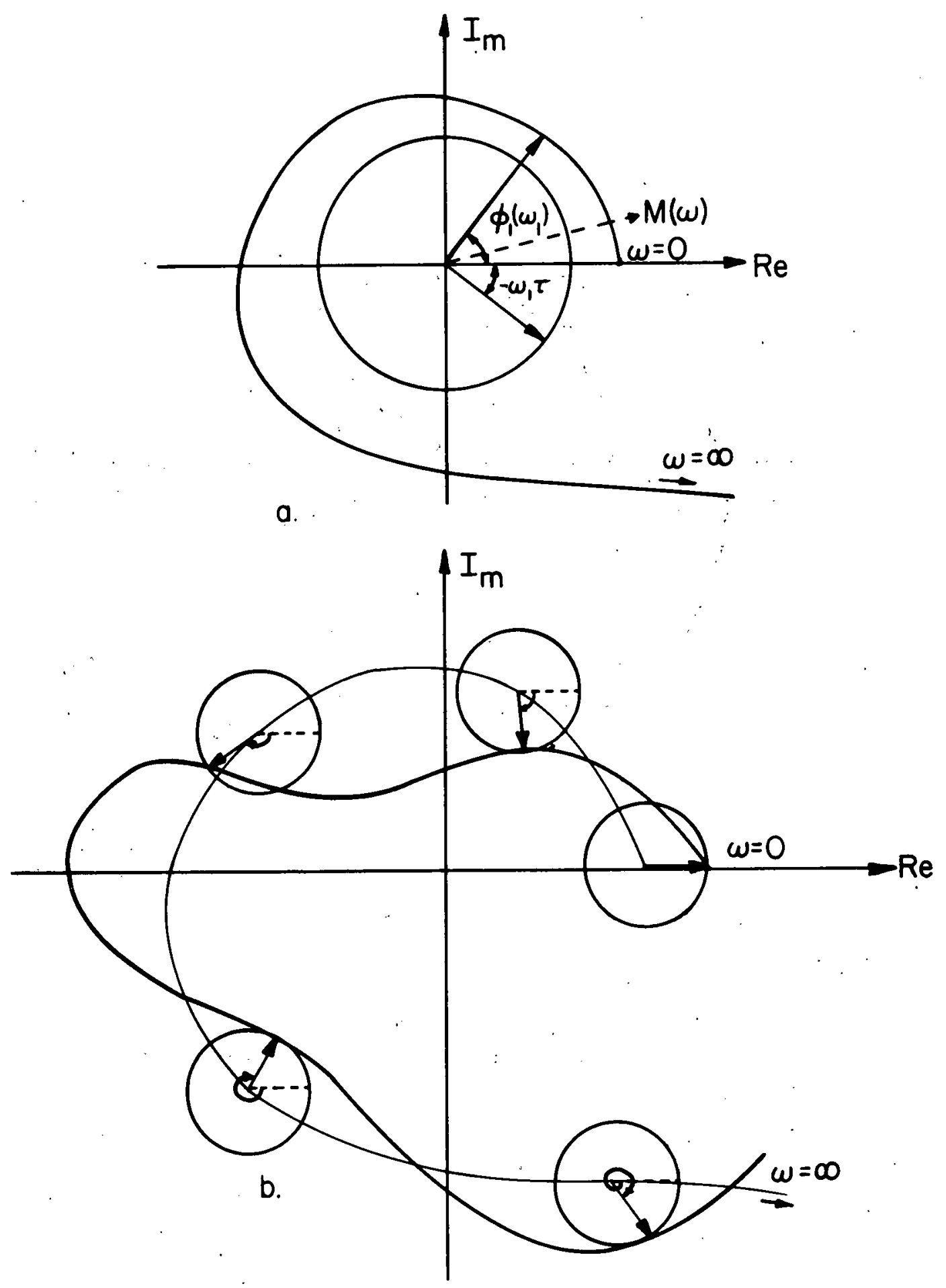

Figure 3.2. Example of Auxiliary Curve and Unit Circle 


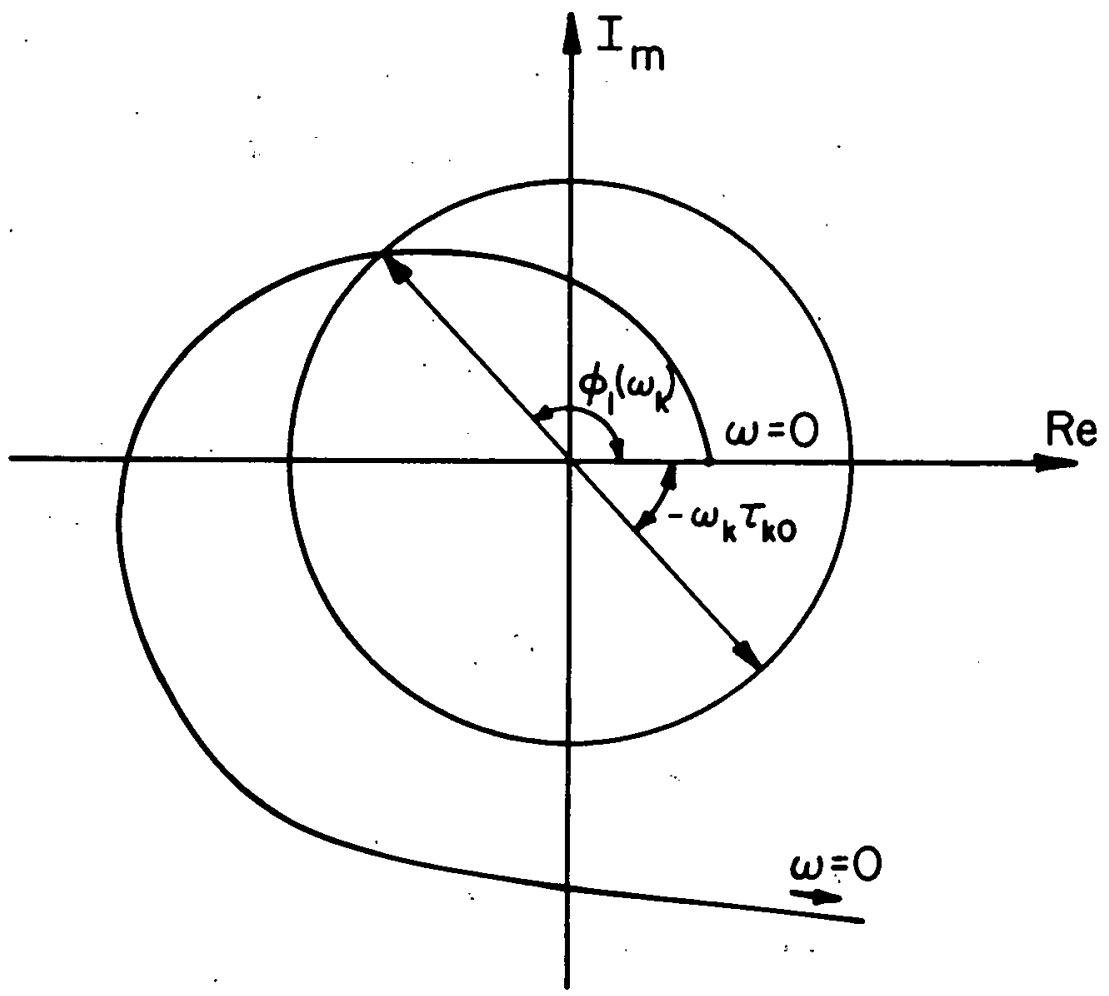

Figure 3.3. Example of Auxiliary Curve and Unit Circle with One Intersection 


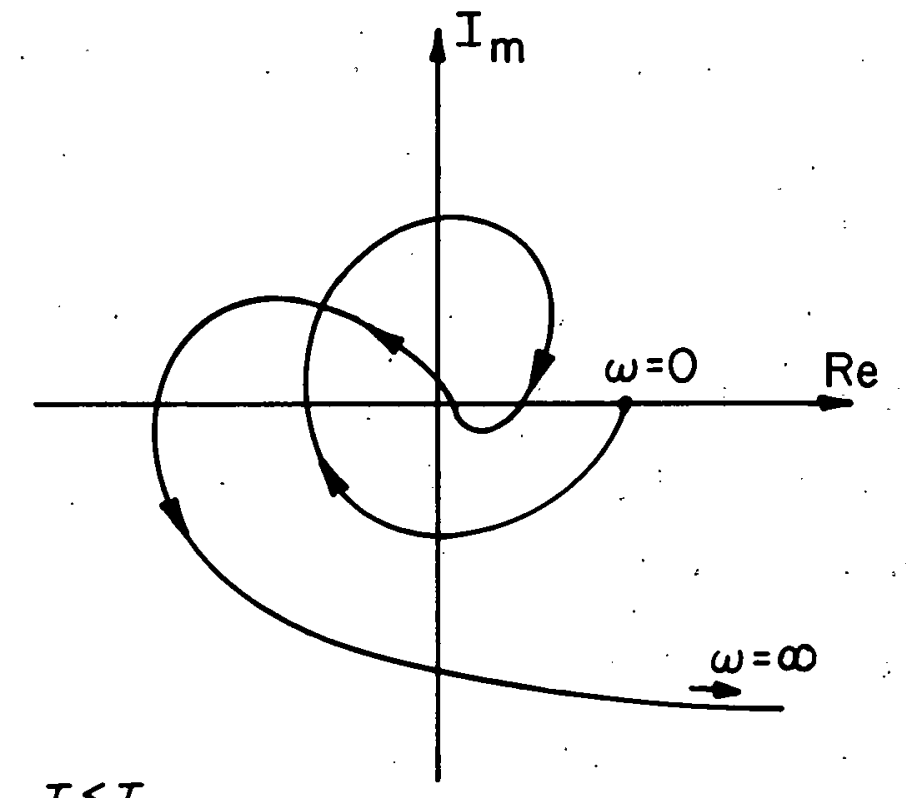

a. $\tau<\tau_{k \downarrow}$

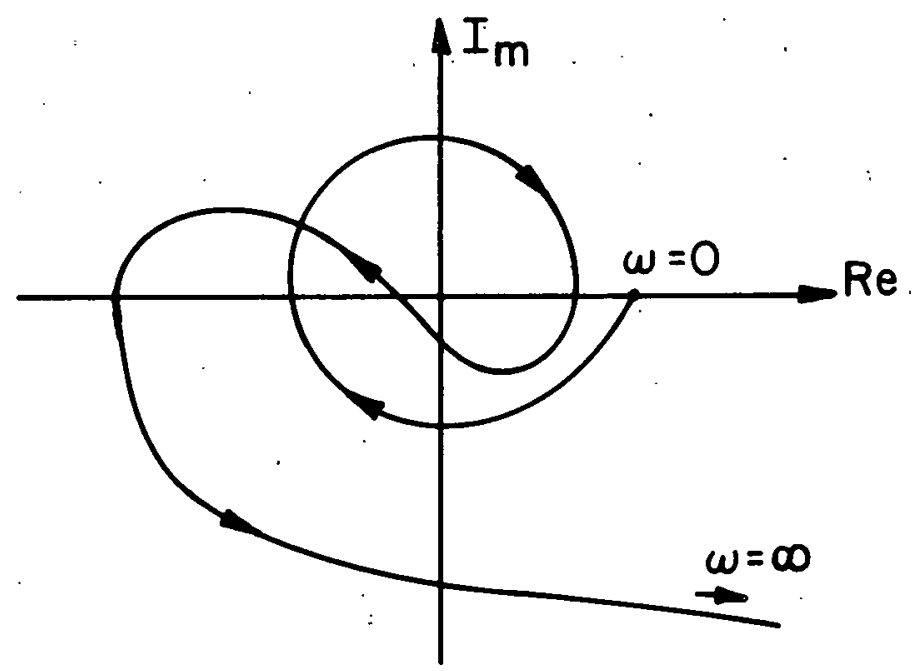

b. $\tau>\tau_{k 1}$

Figure 3.4. Unstable Mikhailov Curves for $\tau$ on Either Side of $\tau_{k l}$ 
In a similar manner, it can be seen that if the system is unstable for $\tau<\tau_{k_{0}}$, then it is unstable for all values of the time delay (Popov 1962, p. 433).

It is instructive to consider the one remaining example shown in Fig. 3.5: The auxiliary curve in this example intersects the unit circle twice. By performing an analysis corresponding to that of Eqs. $(3-27)$ and (3-28), we can find

$$
\begin{aligned}
& \tau_{10}=1 / \omega_{1}\left[\pi-\phi_{1}\left(\omega_{1}\right)\right] \\
& \tau_{20}=1 / \omega_{2}\left[\pi-\phi_{1}\left(\omega_{2}\right)\right] .
\end{aligned}
$$

It is also possible to write

$$
\begin{array}{ll}
\tau_{11}=1 / \omega_{1}\left[\pi-\phi_{1}\left(\omega_{1}\right)+2 \pi\right] & \tau_{21}=1 / \omega_{2}\left[\pi-\phi_{1}\left(\omega_{2}\right)+2 \pi\right] \\
\tau_{12}=1 / \omega_{1}\left[\pi-\phi_{1}\left(\omega_{1}\right)+4 \pi\right] & \tau_{22}=1 / \omega_{2}\left[\pi-\phi_{1}\left(\omega_{2}\right)+4 \pi\right] .
\end{array}
$$

Now since in Fig. 3.5 we have $\omega_{2}>\omega_{1}$ and $\phi_{1}\left(\omega_{2}\right)>\phi_{1}\left(\omega_{1}\right)$, we have

$$
\tau_{20}<\tau_{10}
$$

The behavior of the intersection at $\omega_{2}$ will be the same as the intersection in the previous example (Fig. 3.3). Therefore the system with a delay $\tau<\tau_{20}$ will be stable. With $\tau$ slightly less than $\tau_{10}$ ' it can be seen from Fig. 3.5 that the resultant curve will pass below the origin indicating an unstable system. However, for $\tau$ slightly greater than $\tau_{10}$, the curve will pass above the origin indicating stability. Consequently, in distinction to the previous example, the system will be unstable in the zone $\tau_{20}<\tau<\tau_{10}$ rather than for all 


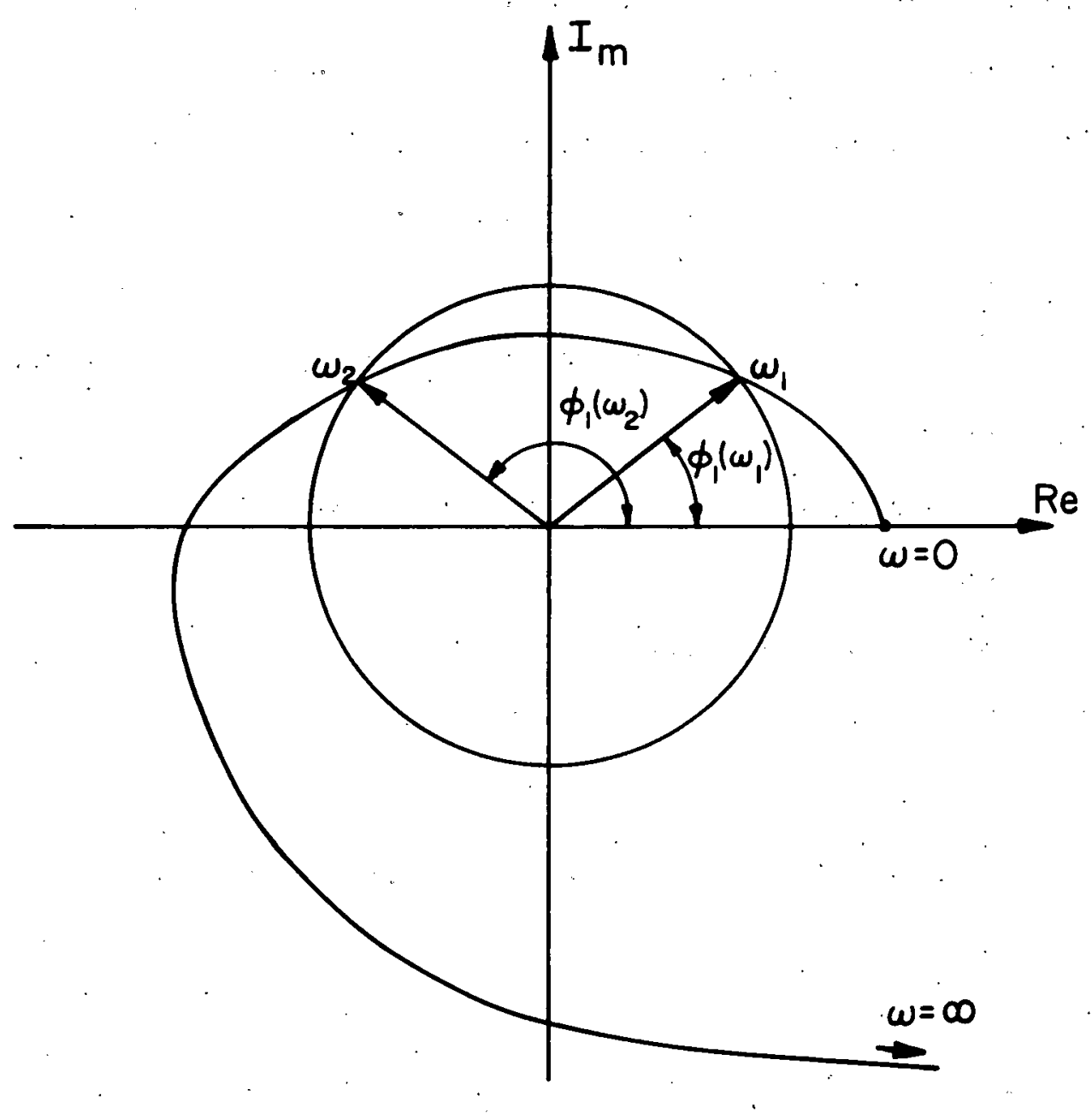

Figure 3.5. Example of Auxiliary Curve and Unit Circle with Two Intersections 
$\tau>\tau_{20}$. With $\tau_{10}<\tau<\tau_{21}$, the system again becomes stable, and there is a continuous alternation of regions of stability and instability corresponding to the wavy nature of the Mikhailov curve with delay. A similar alternation is possible if the system is unstable for $\tau<\tau_{20}$. If at some point it happens that two or more successive values of $T_{1}$ fall between successive values of $\tau_{2}$, then the alternation of stability bands will cease; and the system will probably be unstable for all larger values of $\tau$.

Consider now the characteristic equation developed earlier in Eq. $(2-15)$.

$$
M(s)=Q(s)+R(s) e^{-s \tau}
$$

The equation for the Mikhailov curve is then

$$
M(j \omega)=Q(j \omega)+R(j \omega) e^{-j \omega \tau}
$$

The analysis of this slightly more complicated system proceeds as in the examples above but with the unit circle being replaced by the vector $R(j \omega)$ with the proper rotation by the delay $-\omega \tau$. If we now write $Q$ and $R$ in terms of their moduli and phases, we have

$$
M(j \omega)=M_{1}(\omega) e^{j \phi}(\omega)+M_{2}(\omega) e^{j\left[\phi_{2}(\omega)-\omega \tau\right]}
$$

where $M_{1}(\omega), \phi_{1}(\omega)$ and $M_{2}(\omega), \phi_{2}(\omega)$ represent the moduli and phases of $Q(j \omega)$ and $R(j \omega)$ respectively.

Now in order for the Mikhailov curve to pass through the origin, then for some real $\omega_{k}>0$, the two moduli must be equal, and the phases of the two terms must be oppositely directed. If

$$
M_{1}\left(\omega_{k}\right)=M_{2}\left(\omega_{k}\right) \quad \omega_{k}>0,
$$


then the phase condition requires

$$
\phi_{1}\left(\omega_{k}\right)=\phi_{2}\left(\omega_{k}\right)-\omega_{k} \tau+\pi+2 \pi q \quad q=0,1,2, \ldots \quad \vdots
$$

From Eq. (3-36), we can find the critical values of the time delay, $\tau_{\mathrm{kq}}$ ' for which the Mikhailov curve will pass through the origin.

$$
\tau_{k q}=1 / \omega_{k}\left[\phi_{2}\left(\omega_{k}\right)+\pi-\phi_{1}\left(\omega_{k}\right)+2 \pi q\right] \quad . q=0,1, \ldots
$$

Now since this process only indicates roots on the imaginary axis, some other technique, such as the Modified Mikhailov plot, must be used to determine the stability on either side of the critical value. It should be pointed out that if $\phi_{2}\left(\omega_{k}\right)$ should lead $\phi_{1}\left(\omega_{k}\right)$ by a value $\pi$ or more, then several negative values of $q$ must be included in Eqs. (3-36) and (3-37) in order to include every critical value of the time delay. Physically, this is highly unlikely situation since the order. of $Q$ is generally much larger than the order of $R$. Henceforth, we will assume that $q=0$ gives the minimum positive value of the time delay.

In order to discover the possible existence of regions in which stability is conditional on the value of the time delay, we must search for positive real roots of $\mathrm{Eq} .(3-35)$ as a function of the system parameters. To determine the absolute existence of regions of conditional stability, the procedure is as follows. If Eq. (3-35) has one or more positive real roots, then calculate the critical delays, ' ${ }_{\text {crit }}$. With the aid of the Modified Mikhailov criterion and of the information discussed in the examples above, determine the stability of the system for a time delay slightly less than $\tau_{c r i t}$ and for a time delay slightly greater than $\tau_{\text {crit }}$. Here, we again note that the system can be unstable 
on either side of $\tau_{\text {crit }}$, but not stable on either side. If the system is indeed unstable on both sides, then the calculated value of $\tau_{c r i t}$ is extraneous with regard to stability.

If the system is stable for $\tau=0$, then it is stable for $0<\tau<\tau_{\text {crit-f }}$, where $\tau_{\text {crit-f }}$ is the lowest (fundamental) critical time delay. This approach can often be used to avoid the more complicated Modified Mikhailov plot. Generally, only the fundamental critical delay or the first few stability bands are of interest in physical situations.

\section{Mapping Conditionally Stable Regions} In System Parameter Spaces

In the previous section, we saw how the existence of conditionally stable regions depends on the existence of positive real roots of Eq. (3-35). In this section, we will discuss the determination of positive real roots as functions of the system parameters.

Employing the definition of a modulus of a complex variable, we can write Eq. $(3-35)$ as

$$
\left\{\operatorname{Re}[Q(j \omega)]^{2}+\operatorname{Im}[Q(j \omega)]^{2}\right\}^{\frac{1}{2}}=\left\{\operatorname{Re}[R(j \omega)]^{2}+\operatorname{Im}[R(j \omega)]^{2}\right\}^{\frac{1}{2}} .
$$

Since the modulus of a complex variable is defined to be non-negative, we can square both sides of Eq. (3-38) without losing any information to obtain

$$
\operatorname{Re}[Q(j \omega)]^{2}+\operatorname{Im}[Q(j \omega)]^{2}-\operatorname{Re}[R(j \omega)]^{2}-\operatorname{Im}[R(j \omega)]^{2}=0 .
$$

Now since $R$ and $Q$ are polynomials, $\operatorname{Re}[Q(j \omega)]$ and $\operatorname{Re}[R(j \omega)]$ will be polynomials in even powers of $\omega$ and $\operatorname{Im}[Q(j \omega)]$ and $\operatorname{Im}[R(j \omega)]$ will be 
polynomials in odd powers of $\omega$. The largest order of these polynomials will be $n$, the order of the system. Upon squaring these polynomials, Eq. (3-39) will result in an $\mathrm{n}^{\text {th }}$ order polynomial in $\omega^{2}$.

$$
A_{n}\left(\omega^{2}\right)^{n}+A_{n-1}\left(\omega^{2}\right)^{n-1}+\ldots+A_{1} \omega^{2}+A_{0}=0
$$

Now in order for this equation to give a positive real value for $\omega$ (indicating critical delays), there must be a positive real root for $\omega^{2}$. Therefore, the problem of determining the possible existence of conditionally stable regions in the parameter space reduces to the determination of the positive real roots of an $n^{\text {th }}$ order polynomial as a function of the coefficients where these coefficients are functions of the system parameters.

One of the simplest methods of searching for positive real roots is with Déscartes' rule of signs which can be stated as follows (Archbold 1964, p. 197; Lehnigk 1966, p. 125).

Déscartes' Ruze of Signs. Counting each root according to its multiplicity, the number of positive real roots of a real polynomial is equal to the number $c$ of changes in signs in the sequence of coefficients, or is less than $\mathrm{C}$ by an even number. (Zero coefficients are ignored in counting changes of sign).

This rule as well as the following theorem is used to determine specific zones in the parameter space much as stability zones can be determined by the Routh-Hurwitz criterion.

If the parameters of the system are $\alpha_{1}, \ldots, \alpha_{m}$ such that $A_{\ell}=$. $A_{\ell}\left(\alpha_{1}, \ldots, \alpha_{m}\right)$, Déscartes' rule of signs is used in the following 
manner. Assuming that the $A_{\ell}$ are continuous functions of the parameters, $A_{\ell}$ can only change signs by passing through a zero of $A_{\ell}$ (Buck 1956, p. 41). Therefore if the curves $A_{\ell}=0, \ell=0, \ldots, n$ are drawn in the space of parameters $\alpha_{1}, \ldots, \alpha_{m}$, the parameter space will be divided into regions within which the number of sign changes of Eq. (3-40) remains constant. The number of sign changes can alter only by passing through a zero of one of the coefficients. Generally, in any physically significant region of the system parameter space, not all of the curves $A_{\ell}=0$ will be important. This fact often simplifies the stability analysis.

If the number of sign changes in a region is odd, then the rule of signs guarantees that there will be at least one positive real root of the polynomial. However, if the number of changes is even, then there may or may not be positive real roots because of the "even number less" statement. In fact, a region containing an even number of sign changes can often be divided into subregions, some with and some without positive real roots. Obviously, if this situation occurs in a physically significant region of the parameter space, some other approach must be taken.

If the rule of signs fails to provide the desired information, Sturm's theorem (Archbold 1964, p. 198 ff.; Lehnigk 19.66, p. 105 ff.) may be used to find regions in which positive real roots of Eq. (3-40) exist. Sturm's theorem is based on the Sturm chain which can be described as follows. If $\mathrm{f}(\mathrm{x})$ represents an ordinary real polynomial and $f^{\prime}(x)$ its derivative, then by long division $f(x)$ may be divided by $f^{\prime}(x)$ 
to give a quotient $q_{1}(x)$ and a remainder $-f_{2}(x)$ with the order of $f_{2}(x)$ less than the order of $f_{1}(x)=f^{\prime}(x)$. Thus

$$
f(x)=q_{1}(x) f_{1}(x)-f_{2}(x) .
$$

If $\mathrm{f}_{2}(\mathrm{x})$ is not zero or $\mathrm{a}$ constant, the process is continued by

$$
f_{1}(x)=q_{2}(x) f_{2}(x)-f_{3}(x)
$$

This long division process is known as the negative remainder or Euclid's algorithm. It is continued until the remainder is either zero (indicating that $\mathrm{f}_{\nu}$ is an exact divisor of $\mathrm{f}_{\nu-1}$ ) or a constant: The resulting sequence $\left\{f(x), f_{1}(x), f_{2}(x), \ldots, f_{\nu}(x)\right\}$ is called Sturm's chain. Sturm's theorem can then be stated as follows.

Sturm's Theorem. Let $\rho^{\prime}$ and $\rho^{\prime \prime}, \rho^{\prime}<\rho^{\prime \prime}$, be points at which a real polynomial $\mathrm{f}(\mathrm{x})$ is different from zero. Let $\mathrm{V}(\mathrm{x})$ be the number of variations in sign in Sturm's chain of $f(x)$ at $x$ with zeros not counted. Then the number of distinct zeros of $f(x)$ in the interval $\rho^{\prime}<\mathrm{x}<\rho^{\prime \prime}$ is equal to the number $\mathrm{V}\left(\rho^{\prime}\right)-\mathrm{V}\left(\rho^{\prime \prime}\right)$.

In using this theorem to determine positive real roots, we let $\rho^{\prime}=0$ and $\rho$ " be so large that it is to the right of any real roots.

To demonstrate the use of Sturm's theorem, consider the following example.

$$
f(s)=[s-3][s-(4+j)][s-(4-j)]=s^{3}-11 s^{2}+41 s-51=0
$$

Now Déscartes! rule of signs predicts either one or three $(C=3)$ positive real roots, and hence its results are inconclusive. 
By taking the derivative of $f(s)$ to give $f_{l}(s)$ and by continuing the negative remainder algorithm as indicated above, Sturm's chain can be shown to be

$$
\begin{array}{ll}
f(s)=s^{3}-11 s^{2}+41 s-51 & f_{2}(s)=-(4 / 9) s+8 / 9 \\
f_{1}(s)=3 s^{2}-22 s+41 & f_{3}(s)=-9 .
\end{array}
$$

The sequence at $s=0$ is $\{-51,41,8 / 9,-9\}$ with $V(0)=2$. Similarly, the sequence as $s \rightarrow \infty$ is $\{1,3,-4 / 9,-9\}$ with $V(\infty)=1$. Therefore, the number of positive real roots of $\mathrm{Eq} .(3-43)$ is given by

$$
V(0)-V(\infty)=2-1=1
$$

which is of course evident from Eq. (3-43).

To use this theorem to determine conditionally stable regions in the parameter space, the zeros of the polynomials in the chain $V(0)$ and in the chain $\mathrm{V}\left(\rho^{\prime \prime} \rightarrow \infty\right)$ are plotted in the parameter space. Using similar reasoning to that used with Descartes' rule of signs, the parameter space is divided into regions within which there is a specified number of positive real roots. This number can change only by crossing a zero of one of the polynomials in the chains.

In practice, Sturm's theorem is very difficult to use in analytic form for all but the simplest system. However, drawing from the results of Déscartes' rule of signs, a computer technique to determine regions with positive real roots is easily employed in the physically significant regions of the parameter space. This computer technique, as well as all of the methods discussed in this chapter,' will be demonstrated in example problem in Chapter 4. 


\title{
CHAPTER 4
}

\section{APPLICATION OF THE PARAMETRIC STABILITY ANALYSIS \\ TECHNIQUE TO A THERMIONIC REACTOR MODEL.}

\begin{abstract}
In order to demonstrate the parametric stability analysis techniques discussed in Chapter 3, a seventh-order thermionic reactor model will be treated. After the modeling equations have been presented. with a brief discussion of their development, the model will be analyzed for regions of conditional stability; and critical time delays will be calculated. In addition, the system stability under the zero-delay, infinite-delay, and Taylor series approximations will be determined for comparison with the exact techniques; and a discussion of the physical processes leading to regions of conditional stability in the parameter space will be given.
\end{abstract}

The Thermionic Reactor Model

Several authors have studied the stability problems associated with the in-core thermionic reactor concept (Gronroos 1967; Brehm, et. al. 1968; Brehm, Hetrick, and Schmidt 1969; Guppy, 1970a). With the in-core concept, heat energy from fission is supplied directly to a metallic surface (emitter) causing electrons to gain enough energy to leave the surface, traverse a cesiated gap, and be collected by a second metallic surface called a collector. The electrons then return to the emitter through an electrical load producing the useful work. 
The proposed configuration of an in-core thermionic diode consists of a cylindrical fuel slug with a cylindrical fuel cladding doubling as the emitter surface. A small gap filled with cesium surrounds the emitter which is in turn surrounded by concentric cylinders of the collector material, electrical insulator, and outer cladding material. Ideal thermal contacts between materials are assumed. A number of these diodes are connected in series and are placed one on top of another in a "flashlight" fashion to form a fuel assembly. A diode with these series connections is shown in Fig. 4.1.

The fast reactor core is formed by a number of such fuel assemblies which are electrically connected in parallel and series in a manner to meet specified power conditioning and redundancy requirements. A coolant flows past each stack of diodes transporting the waste heat to a radiator where it is rejected to space. This entire system is shown schematically in Fig. 4.2.

As Brehm, et. al. (1969) have pointed out, the principal feedback mechanisms arise from Doppler-effect contributions from the various regions shown in Fig. 4.1, as well as from expansion effects. With respect to power changes, the in-core thermionic reactor is expected to have a region of prompt, positive reactivity feedback (from the positive Doppler of the fuel), followed by regions of successively delayed (not in a pure sense) negative feedbacks. Previous studies have been directed to determining the ranges of conditions, principally the ranges of temperature coefficients of reactivity, under which the system is stable. As was done previously, we assume that all diodes in the core. may be typically represented by one diode. Following Brehm et. al. 


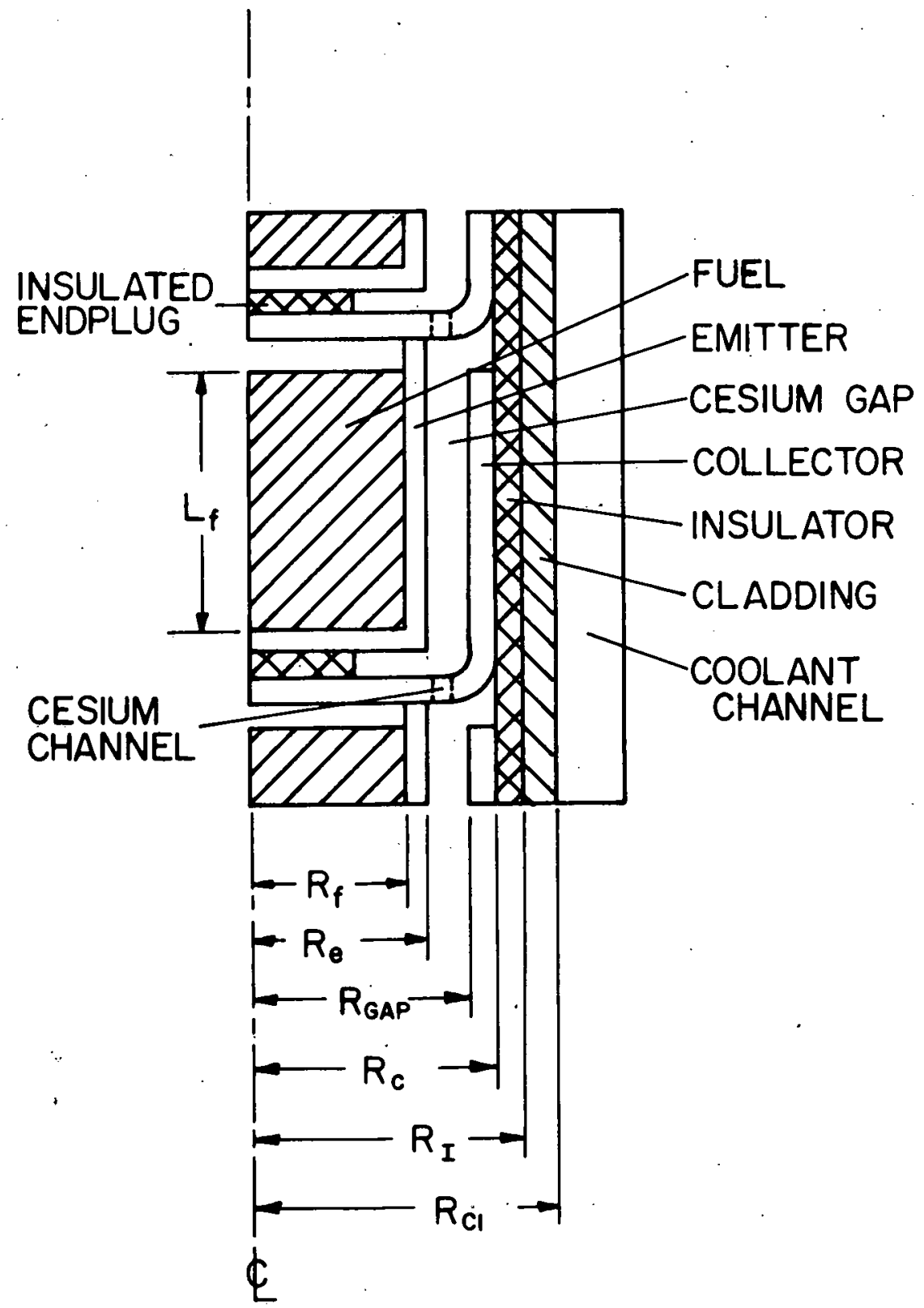

Figure 4.1. Typical Representation of Series-Connected In-Core Thermionic Diode in "Flashlight" Configuration 


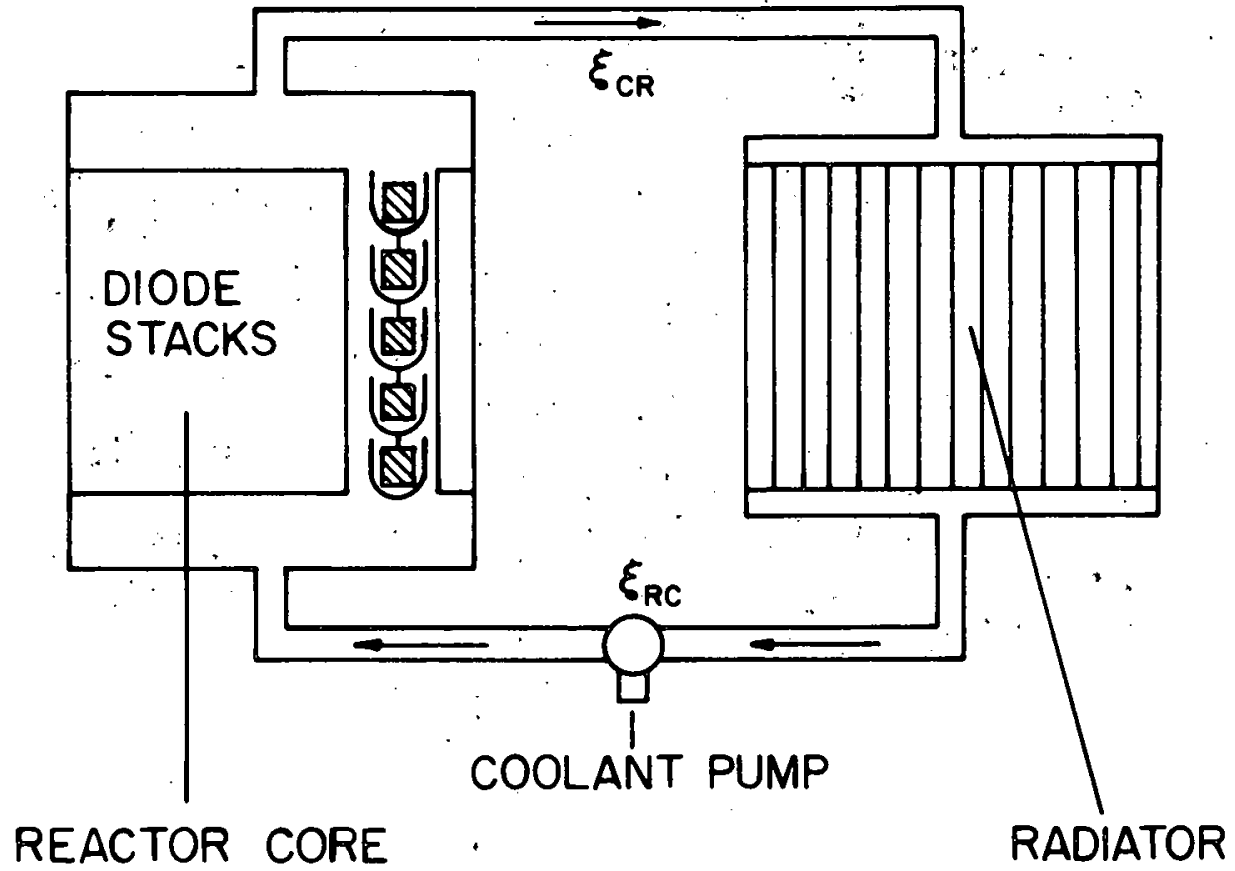

Figure 4.2. Thermionic Reactor System Schematic 
(1969) we then use the prompt-jump approximation to the one-delayedgroup point-reactor equations to describe the neutron dynamics. Rather than writing one heat transfer equation for each region as Gronroos (1967) and Guppy (1970a) have done, we assume that the fuel and emitter may be treated as a single region described by the emitter temperature. Similarly, we treat the collector-insulator-clad region as an entity described by the average insulator temperature. Brehm, et. al. (1969) have discussed the validity of these groupings with regard to a temperature feedback stability analysis.

Previous models used in the dynamic analysis of thermionic reactor systems have used either the constant-coolant-inlet-temperature $(\tau=\infty)$ approximation (Brehm, et. al. 1969; Brehm, et. al. 1968) or the Taylor series approximation (Gronroos 1967; Guppy 1970a). In the model for this analysis, we will go one step further by representing the coolant loop with pure time delays between the reactor and radiator.

With the above introductory comments, we may now begin to write the system model. The prompt-jump equation is written

$$
\dot{\mathrm{n}}=\frac{1}{\beta-\rho}(\lambda \rho+\dot{\rho}) \mathrm{n}
$$

where the dot denotes the time derivative. The other symbols are defined in Appendix A. The feedback reactivity is given by

$$
\rho=-\alpha_{1}\left(T_{1}-T_{10}\right)-\alpha_{2}\left(T_{2}-T_{20}\right)
$$

where $T_{1}$ and $T_{2}$ represent the temperatures of the fuel-emitter and collector-insulator-clad regions respectively, and where the subscript 0 denotes the equilibrium value. The symbols $\alpha_{1}, \alpha_{2}$ represent the 
temperature coefficients of reactivity for regions one and two. Here we use the convention that positive values for $\alpha_{1}, \alpha_{2}$ indicate negative feedback.

Assuming uniform heat generation in the fuel and no heat generation in the other components, we can take heat balances on the fuel and emitter regions to give

$$
\begin{aligned}
& \rho_{f} V_{f} C_{\rho_{f}} \dot{T}_{f}=V_{f} n-\frac{4 \pi\left(T_{f}-T_{e}\right)}{\left[\frac{1}{2 k_{f} L_{f}}+\frac{10 g\left(R_{e} / R_{f}\right)}{k_{e} L_{e}}\right]} \\
& \rho_{e} V_{e} C_{\rho} \dot{T}_{e}=\frac{4 \pi\left(T_{f}-T_{e}\right)}{\left|\frac{1}{2 k_{f} L_{f}}+\frac{\log \left(R_{e} / R_{f}\right)}{k_{e} L_{e}}\right|}-2 \pi R_{e} L_{e} q_{e}
\end{aligned}
$$

The definitions of the symbols are given in Appendix $A . T_{f}$ is an average fuel temperature determined by the following volume average.

$$
T_{f}=1 / V_{f} \int_{0}^{R_{f}} 2 \pi r L_{f} T_{f}^{\prime}(r) d r=T_{f}^{\prime}(0)-\frac{n R_{f}^{2}}{8 k_{f}} .
$$

$R_{f}$ and other radii are shown in Fig. 4.1. Since the emitter and outer regions are relatively thin, linearly averaged temperatures are used. The average temperature for the emitter, for example, is given by

$$
T_{e}=\frac{1}{2}\left[T_{f}^{\prime}\left(R_{f}\right)+T_{e}^{\prime}\left(R_{e}\right)\right]
$$

Several different processes combine to carry heat from the emitter across the cesiated gap to the collector. The combination of these processes are represented in $\mathrm{q}_{e}$. Of the total energy transferred. across the gap, generally $60 \%$ or more is carried by electrons (electron cooling) while the remaining energy is transmitted by radiation, 
conduction, or by end and lead losses (Brehm, et. al. 1969). At the collector, $10 \%$ to $20 \%\left(n_{e}\right)$ of the energy is carried to the load while the remaining waste heat is conducted to the coolant.

Although the processes making up $\mathrm{q}_{\mathrm{e}}$ are highly nonlinear, it has been observed (Gronroos, 1967) that the total heat flow may be approximated quite well by a linear representation

$$
q_{e}=H\left(T_{e}-T_{b}\right)
$$

where $T_{b}$ is an empirical constant and $H$ is determined from the steady state condition

$$
H=\frac{R_{f}^{2} L_{f} n_{0}}{2 L_{e} R_{e}\left[T_{e 0}-T_{b}\right]} .
$$

With the computer program SIMCON (Wilkins 1968), Guppy (1970b) has calculated the emitter heat flow versus emitter temperature for the conditions assumed in this study. The results are shown in Fig. 4.3. If $\mathrm{T}_{\mathrm{e} 0}$ is taken to be approximately $2000^{\circ} \mathrm{K}$, then it can be determined from Fig. 4.3 that $\mathrm{T}_{\mathrm{b}}=1445^{\circ} \mathrm{K}$.

Now since we wish to treat the fuel and emitter as a single region, the resultant heat transfer description is found by adding Eqs. (4-3) and (4-4). Then under the assumption that

$$
\dot{\mathrm{T}}_{\mathrm{f}}=\dot{\mathrm{T}}_{\mathrm{e}}=\dot{\mathrm{T}}_{1}
$$

and

$$
\mathrm{T}_{1}=\mathrm{T}_{\mathrm{e}}
$$


62

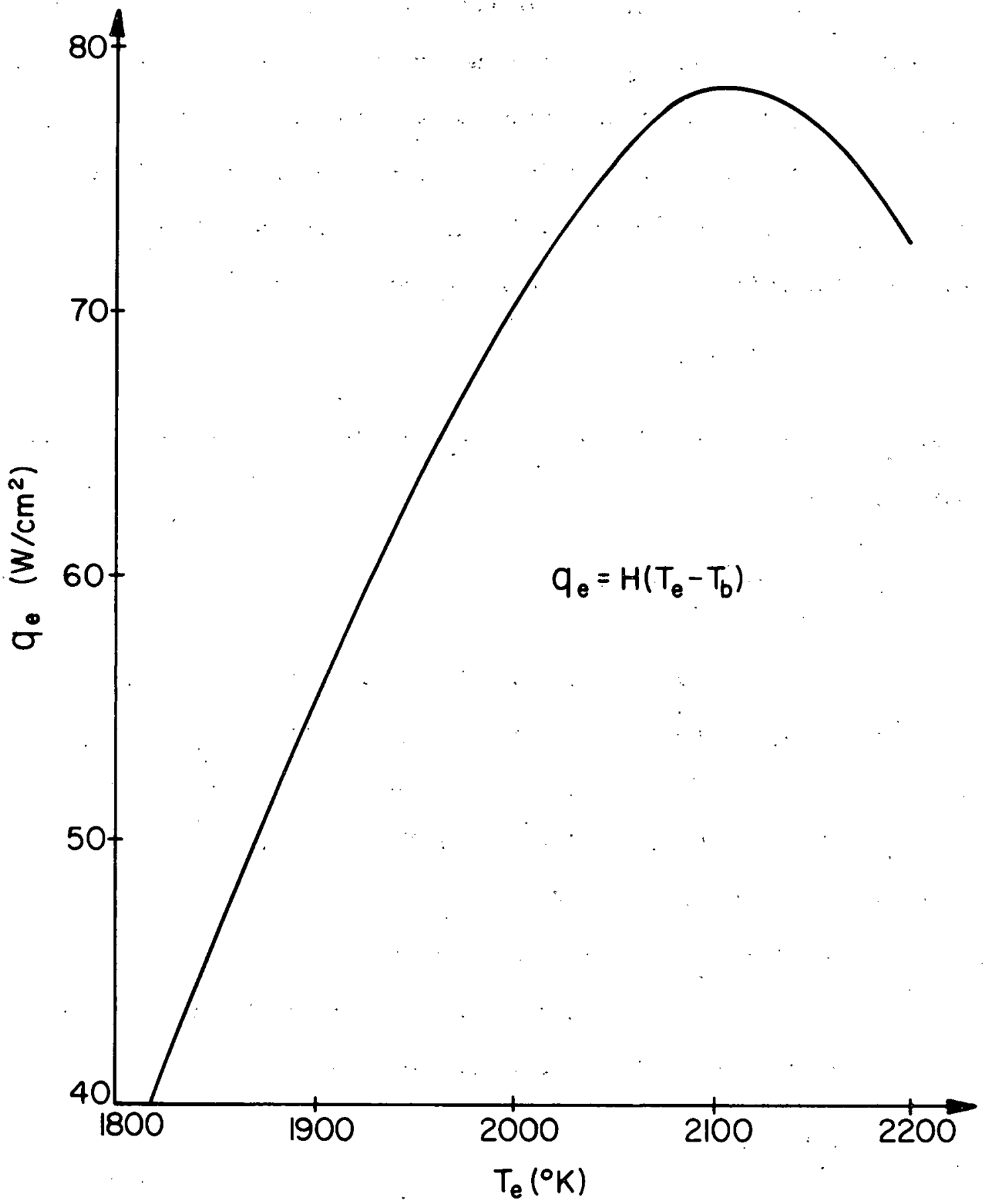

Figure 4.3. Emitter Heat Flow versus Emitter Temperature 
we have

$$
\dot{\mathrm{T}}_{1}=\mathrm{bn}-\mathrm{n}_{1}\left(\mathrm{~T}_{1}-\mathrm{T}_{\mathrm{b}}\right)
$$

where

$$
\begin{gathered}
b=\frac{v_{f}}{\rho_{f} V_{f} C_{p_{f}}+\rho_{e} V_{e} c_{p_{e}}} \\
n_{l}=\frac{2 \pi R_{e} L_{e}}{\rho_{f} V_{f} C_{p_{f}}+\rho_{e} V_{e} C_{p_{e}}}
\end{gathered}
$$

In a manner similar to the fuel-emitter region, the collectorinsulator-clad region can be described by

$$
\dot{\mathrm{T}}_{2}=\eta_{2}\left(\mathrm{~T}_{1}-\dot{\mathrm{T}}_{\mathrm{b}}\right)-\eta_{3}\left(\mathrm{~T}_{2} \cdot-\mathrm{T}_{\mathrm{co}}\right)
$$

where

$$
\begin{aligned}
& \eta_{2}=\frac{2 \pi R_{e} L_{e}\left(1-n_{e}\right) H}{\rho_{c} V_{c} c_{p_{c}}+\rho_{I} V_{I} C_{p_{I}}+\rho_{c I} V_{c l} C_{p_{c l}}} \\
& n_{3}=\frac{4 \pi}{\left[\rho_{c} v_{c} c_{p_{c}}+\rho_{I} V_{I} C_{p_{I}}+\rho_{c L} v_{c L} c_{p_{c L}} \mid\left[\frac{\log \left(R_{s} / R_{c}\right)}{K_{I} L_{I}}+\frac{\log \left(R_{c l} / R_{s}\right)}{K_{c I} L_{c l}}+\frac{2}{h R_{c 1} L_{c l}}\right]\right.} . \\
& \text { (4-16) }
\end{aligned}
$$

-To simulate the coolant flow through the core and radiator, we use a method described by Ball (1964). We assume that the coolant in the channel about a diode stack may be represented by two "well stirred tanks" in series as shown schematically in Fig. 4.4. In addition, we make the additional assumptions that 


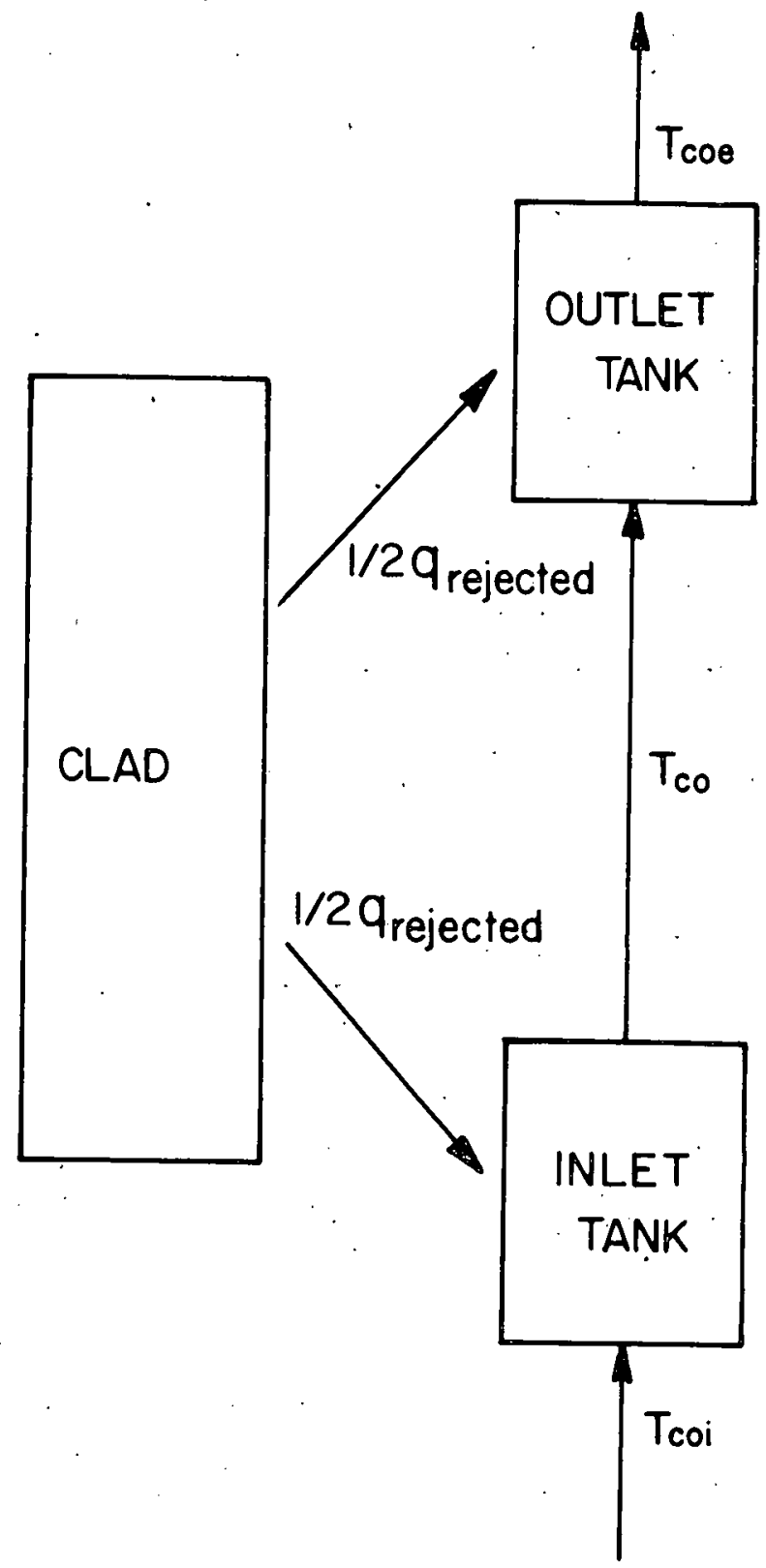

Figure 4.4. Schematic Representation of "TwoWell-Stirred-Tanks" Approximation 
1) the conductivity of the outer clad is infinite in the direction of flow,

2) heat transfer between the clad and coolant is proportional to the mean temperature in the inlet tank,

3) the mean temperatures of the tanks are approximated by their outlet temperatures, and

4) one half of the rejected waste heat flows into each tank.

Under these assumptions, we can write for the inlet tank

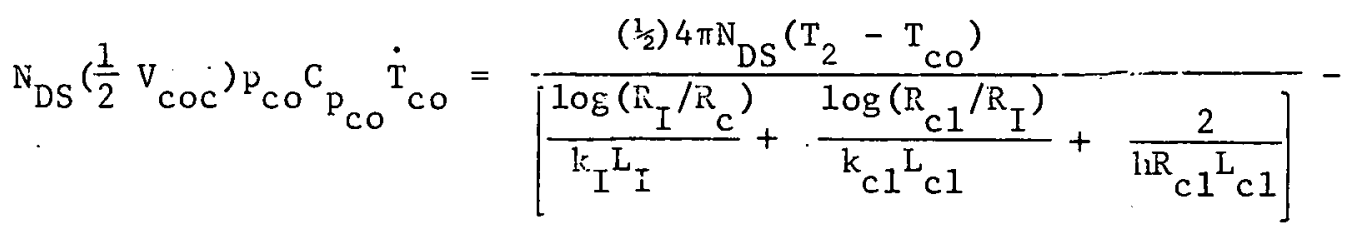

$$
\begin{aligned}
& -w_{\operatorname{coc}} C_{F_{C O}}\left(T_{c o}-T_{c o i}\right)
\end{aligned}
$$

with

$$
\mathrm{T}_{\text {co }}=\frac{1}{2}\left(\mathrm{I}_{\text {coi }}+\mathrm{T}_{\mathrm{coe}}\right)
$$

Rewriting Eq. (4-17), we have

$$
\dot{\mathrm{T}}_{\text {co }}=\eta_{4}\left(\mathrm{~T}_{2}-\mathrm{T}_{\mathrm{co}}^{\prime}\right)-\eta_{5}\left(\mathrm{~T}_{\mathrm{co}}-\mathrm{T}_{\mathrm{coi}}\right)
$$

with

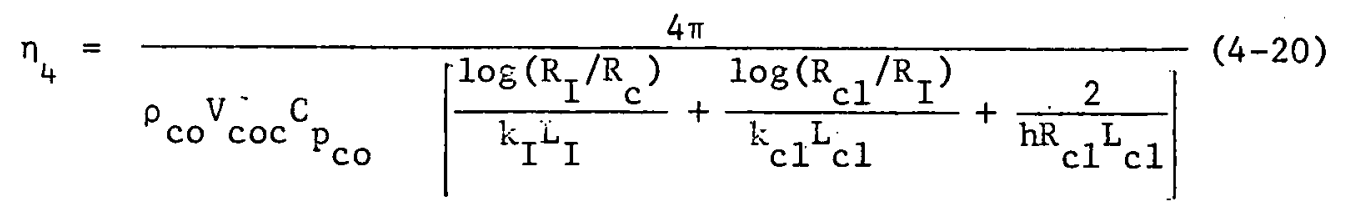

and

$$
n_{5}=\frac{2 \omega \operatorname{coc}}{N_{D S^{\rho} \operatorname{co} V_{c o c}}} \text {. }
$$

Similarly, for the outlet tank, we have 


$$
\dot{\mathrm{T}}_{\text {coe }}=\eta_{4}\left(\mathrm{~T}_{2}-\mathrm{T}_{\mathrm{co}}\right)-\eta_{5}\left(\mathrm{~T}_{\text {coe }}-\mathrm{T}_{\mathrm{co}}\right)
$$

For the description of the coolant passage through the radi- . ator, we again assume the "two-we11-stirred-tanks" approximation. We can then write the following three equations.

$$
\begin{aligned}
& \frac{1}{2} \rho_{C O} V_{c o R} C_{p_{C O}} \dot{T}_{R}=\left(N_{D P} / N_{R P}\right) \omega_{C O C} C_{p_{C O}}\left(T_{R i}-T_{R}\right)-\frac{1}{2} h A_{I N}\left(T_{R}-T_{R P}\right) \\
& \text { (4-23) } \\
& \frac{1}{2} \rho_{C O} V_{C O R} C_{P_{C O}} \dot{T}_{R e}=\left(N_{D P} / N_{R P}\right) \omega_{C O C} C_{P_{C O}}\left(T_{R}-T_{R e}\right)-\frac{1}{2} h A_{I N}\left(T_{R}-T_{R P}\right) \\
& (4-24) \\
& \rho_{R P} V_{R P} C_{P_{R P}} \dot{T}_{R P}=h A_{I N}\left(T_{R}-T_{R P}\right)-\varepsilon_{r} \sigma A_{R S} T_{R P}^{4}
\end{aligned}
$$

If we now make the additional assumptions that $\dot{\mathrm{T}}_{R}=\dot{\mathrm{T}}_{\mathrm{RP}}$ and $\mathrm{T}_{R P} \simeq \mathrm{T}_{\mathrm{R}}$, we obtain; upon adding Eq. (4-25) to each of Eqs. (4-23) and (4-24),

$$
\begin{aligned}
& \dot{\mathrm{T}}_{R}=\eta_{6}\left(\mathrm{~T}_{\mathrm{Ri}}-\mathrm{T}_{R}\right)-\eta_{7}^{\prime} \mathrm{T}_{\mathrm{R}}^{4} \\
& \dot{\mathrm{T}}_{\mathrm{Re}}=\eta_{6}\left(\mathrm{~T}_{\mathrm{R}}-\mathrm{T}_{\mathrm{Re}}\right)-\eta_{7}^{\prime} \mathrm{T}_{\mathrm{R}}^{4}
\end{aligned}
$$

where

$$
\begin{aligned}
& n_{\sigma^{\prime}}=\frac{{ }^{2 N_{D P}{ }^{\omega}} \operatorname{coc}{ }^{C} p_{c o}}{N_{R P}\left[\rho_{c o} V_{c o R}{ }^{C} p_{c o}+{ }_{R P} V_{R P} C_{F_{R P}}\right]} . \\
& \eta_{7}^{\prime}=\frac{\varepsilon_{\mathrm{r}}^{\sigma \mathrm{A}} \mathrm{RS}_{\mathrm{CO}}}{\rho_{\mathrm{CO}} \mathrm{V}_{\mathrm{coR}} \mathrm{C}_{\mathrm{p}_{\mathrm{CO}}}+\rho_{\mathrm{RP}} \mathrm{V}_{\mathrm{RP}} \mathrm{C}_{\mathrm{p}_{\mathrm{RP}}}} .
\end{aligned}
$$

The radiator and the core are coupled by the coolant lagging

terms

$$
\begin{aligned}
& T_{R i}=T_{c o e}\left(t-\xi_{C R}\right) \\
& T_{\text {coi }}=T_{R e}\left(t-\xi_{R C}\right)
\end{aligned}
$$


where $\xi_{C R}$ represents the coolant time delay from the core to the radiator and $\xi_{\mathrm{RC}}$ represents the delay from the radiator to the core. Therefore, the system is described by Eqs. (4-1), (4-11), (4-14), $(4-19),(4-22),(4-26)$, and $(4-27)$.

$$
\begin{aligned}
& \dot{\mathrm{n}}=\frac{1}{\beta-\rho}(\lambda \rho+\dot{\rho}) \mathrm{n}=-\alpha_{1}\left(\mathrm{~T}_{1}-\mathrm{T}_{10}\right)-\alpha_{2}\left(\mathrm{~T}_{2}-\mathrm{T}_{20}\right) \\
& \dot{\mathrm{T}}_{1}=\mathrm{bn}-\eta_{1}\left(\mathrm{~T}_{1}-\mathrm{T}_{\mathrm{b}}\right) \\
& \dot{\mathrm{T}}_{2}=\eta_{2}\left(\mathrm{~T}_{1}-\mathrm{T}_{\mathrm{b}}\right)-\eta_{3}\left(\mathrm{~T}_{2}-\mathrm{T}_{c o}\right) \\
& \dot{\mathrm{T}}_{c o}=\eta_{4}\left(\mathrm{~T}_{2}-\mathrm{T}_{c o}\right)-\eta_{5}\left[\mathrm{~T}_{c o}-\mathrm{T}_{\mathrm{Re}}\left(\mathrm{t}-\xi_{\mathrm{RC}}\right)\right] \\
& \dot{\mathrm{T}}_{c o e}=\eta_{4}\left(\mathrm{~T}_{2}-\mathrm{T}_{c o}\right)-\eta_{5}\left(\mathrm{~T}_{c o e}-\mathrm{T}_{\mathrm{Co}}\right) \\
& \dot{\mathrm{T}}_{\mathrm{R}}=\eta_{6}\left[\mathrm{~T}_{\mathrm{coe}}\left(\mathrm{t}-\xi_{\mathrm{CR}}\right)-\dot{\mathrm{T}}_{\mathrm{R}}\right]-\eta_{7}^{\prime} \mathrm{T}_{\mathrm{R}}^{4} \\
& \dot{\mathrm{T}}_{\mathrm{Re}}=\eta_{6}\left(\mathrm{~T}_{\mathrm{R}}-\mathrm{T}_{\mathrm{Re}}\right)-\eta_{7}^{\prime} \mathrm{T}_{\mathrm{R}}^{4}
\end{aligned}
$$

If, by setting the derivatives of these equations to zero, we determine the equilibrium values of the state variables, and if we make the following definitions,

$$
\begin{array}{ll}
\mathrm{x}_{1}=\mathrm{n}-\mathrm{n}_{0} & \mathrm{x}_{5}=\mathrm{T}_{\text {coe }}-\mathrm{T}_{\text {coe } 0} \\
\mathrm{x}_{2}=\mathrm{T}_{1}-\mathrm{T}_{10} & \mathrm{x}_{6}=\mathrm{T}_{\mathrm{R}}-\mathrm{T}_{\mathrm{R} 0} \\
\mathrm{x}_{3}=\mathrm{T}_{2}-\mathrm{T}_{20} & \mathrm{x}_{7}=\mathrm{T}_{\mathrm{Re}}-\mathrm{T}_{\mathrm{Re}_{0}} \\
\mathrm{x}_{4}=\mathrm{T}_{\text {co }}-\mathrm{T}_{\text {co } 0} &
\end{array}
$$

we can write the linearized system equations in the form of Eq. (2-7).

$$
\dot{\vec{x}}=\overrightarrow{A x}+B_{1} \vec{x}\left(t-\xi_{R C}\right)+B_{2} \vec{x}\left(t-\xi_{C R}\right)
$$


The matrices $A_{,} B_{1}$, and $B_{2}$ are given in the following equations.

$$
\begin{aligned}
& B_{1}=\left[b_{1, i j}\right] \text { with } \\
& b_{1,47}=n_{5} \\
& b_{1, i j}=0 i \neq 4, j \neq 7 \\
& B_{2}=\left[b_{2, i j}\right] \text { with } \\
& b_{2,65}=n_{6} . \\
& b_{2, i j}=0 . i \neq 6, j \neq 5
\end{aligned}
$$




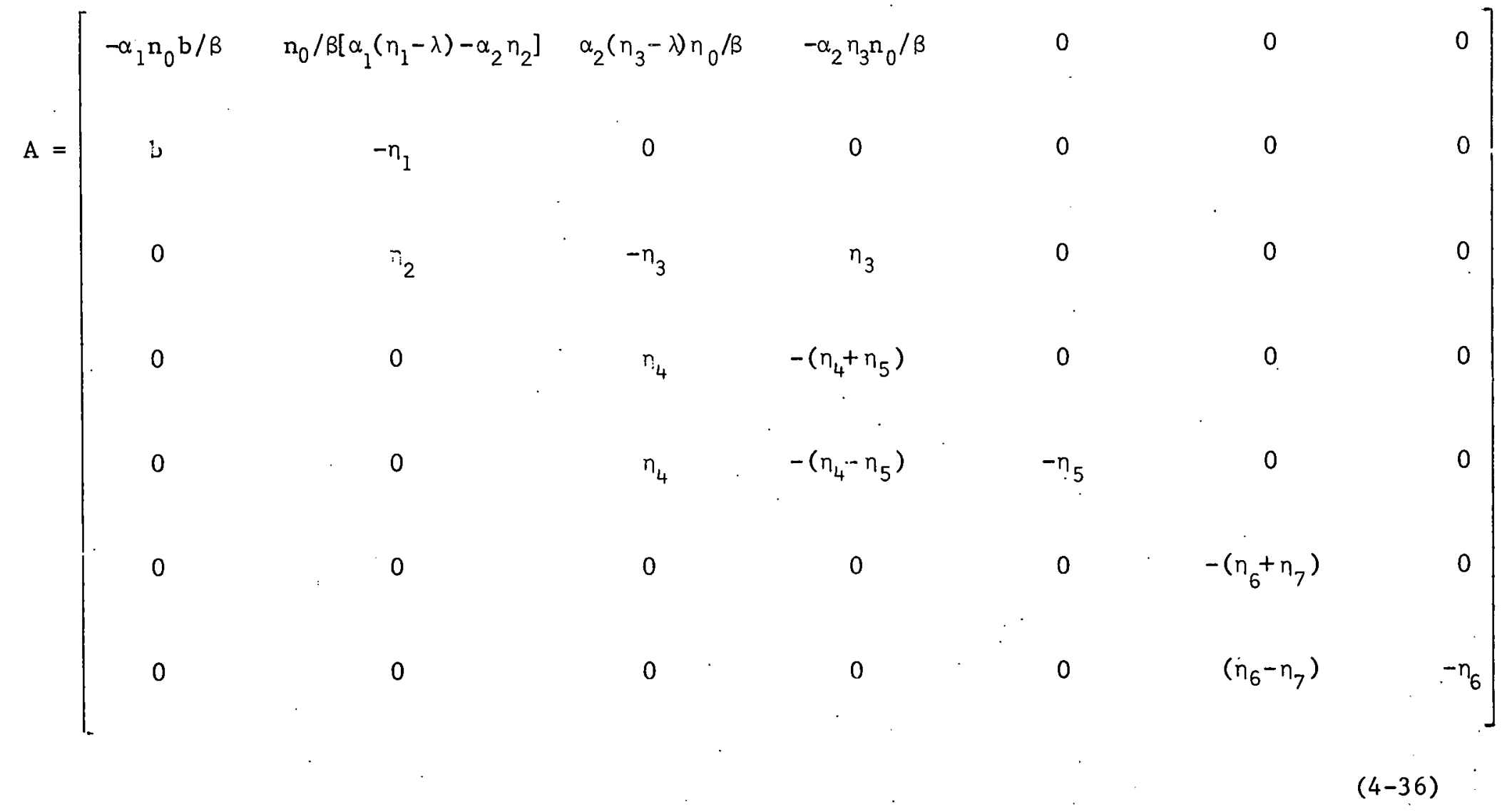


When linearizing the radiative heat loss terms in Eq. (4-31), we made the additional definition

$$
\begin{aligned}
& n_{7}=4 \mathrm{~T}_{\mathrm{RO}}^{3} \eta_{7}^{\prime} \\
& \text { The Characteristic Equation and }
\end{aligned}
$$

The $\left(\omega^{2}\right)^{n}$ Polynomial

In the subsequent analysis of the model presented above, we will be interested in system stability as it is affected by the values of the temperature coefficients of reactivity $\alpha_{1}, \alpha_{2}$ and by the values of the time delay. Therefore, when determining the characteristic equation, we will keep these parameters distinct while the other parameters will be combined as necessary.

From Eq. (4-33), we can write the characteristic determinant by the following equation. 


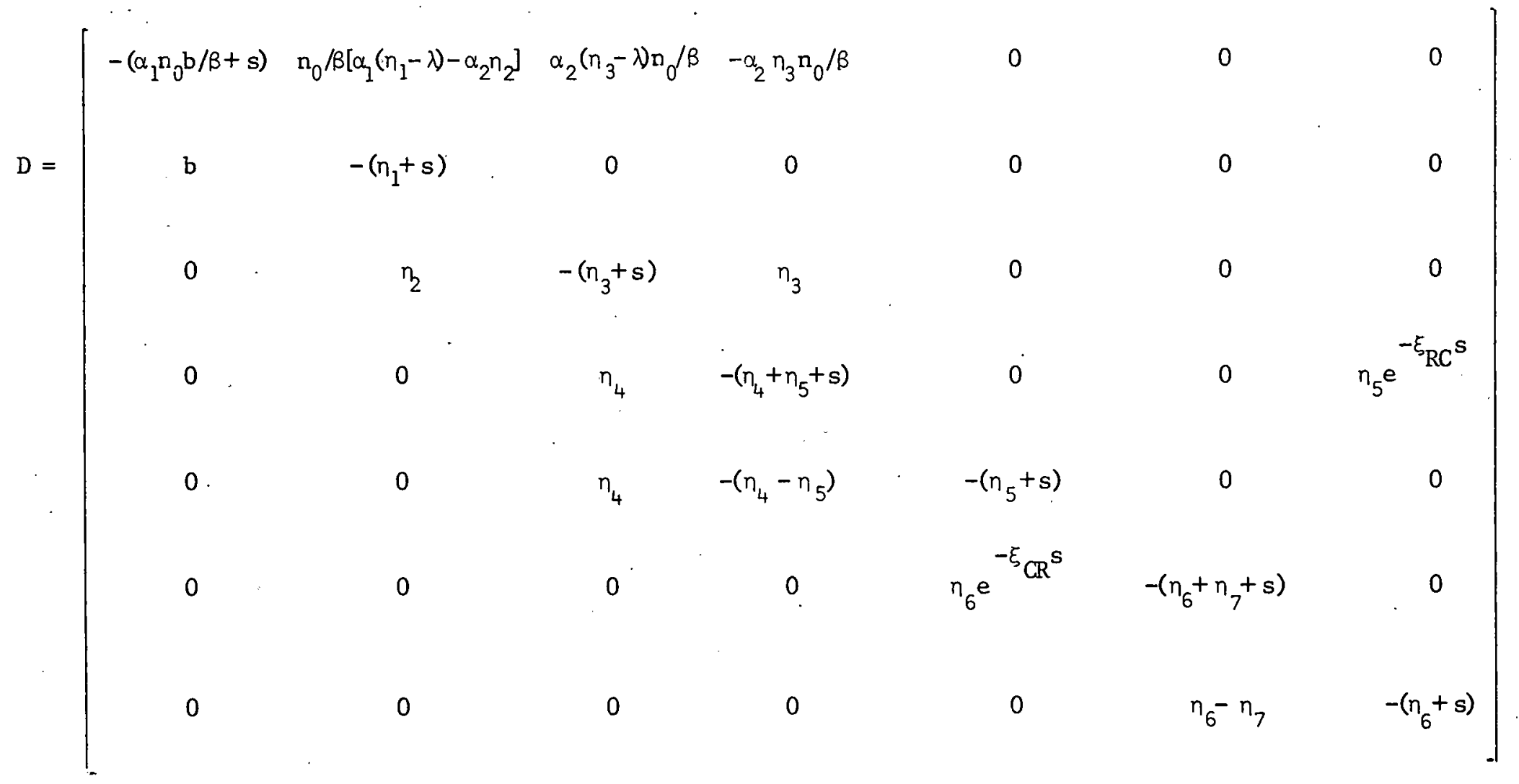

$(4-38)$ 
Evaluating this determinant gives the characteristic equation in the form of Eq. (2-15).

$$
M(s)=Q(s)+R(s) e^{-s \tau}
$$

where

$$
\tau=\xi_{\mathrm{CR}}+\xi_{\mathrm{RC}}
$$

In Eq. $(4-39), \alpha_{1}$ and $\alpha_{2}$ always appear multiplied by $n_{0} b / \beta$.

Therefore, we make the following defiritions

$$
\begin{aligned}
& \gamma_{1}=\alpha_{1} n_{0} b / \beta \\
& \gamma_{2}=\alpha_{2} n_{0} b / \beta .
\end{aligned}
$$

The subsequent stability analysis will be in relation to these newly defined parameters. For Eq. (4-39), $Q(s)$ and $R(s)$ are given by

$$
\begin{aligned}
Q(s)=s^{7} & +\left(\gamma_{1}+C_{10}\right) s^{6}+\left(\gamma_{1} C_{11}+\gamma_{2} \eta_{2}+C_{12}\right) s^{5}+\left(\gamma_{1} C_{13}+\gamma_{2} C_{14}+C_{15}\right) s^{4}+ \\
& +\left(\gamma_{1} C_{16}+\gamma_{2} C_{17}+C_{18}\right) s^{3}+\left(\gamma_{1} C_{19}+\gamma_{2} C_{20}+C_{21}\right) s^{2}+ \\
& +\left(\gamma_{1} C_{22}+\gamma_{2} C_{23}+C_{24}\right) s+\left(\gamma_{1} C_{25}+\gamma_{2} C_{26}\right) \\
R(s)=C_{27} s^{3}+\left(\gamma_{1} C_{27}+C_{29}\right) s^{2}+\left(\gamma_{1} C_{30}+\gamma_{2} C_{31}+C_{32}\right) s & \\
& +\left(\gamma_{1} C_{33}+\gamma_{2} C_{34}\right)
\end{aligned}
$$

where the $C_{i}$ are constants representing various combinations of the system parameters. They are defined in Appendix B.

By setting $s=j \omega$ in Eq. $(4-39)$ and by following the procedure outlined in Chapter 3 for obtaining Eq. (3-40), this system yields 


$$
\begin{aligned}
\left(\omega^{2}\right)^{7} & +A_{6}\left(\omega^{2}\right)^{6}+A_{5}\left(\omega^{2}\right)^{5}+A_{4}\left(\omega^{2}\right)^{4}+A_{3}\left(\omega^{2}\right)^{3}+ \\
& +A_{2}\left(\omega^{2}\right)^{2}+A_{1}\left(\omega^{2}\right)+A_{0}=0 .
\end{aligned}
$$

As was discussed in Chapter 3 , positive real roots of $\omega^{2}$ indicate critical time delays and the possibility of conditionally stable regions. The definitions of the coefficients are given by

$$
\begin{aligned}
& A_{6}=\gamma_{1}^{2}+\gamma_{1} F_{1}+\gamma_{2} F_{2}+F_{3} \\
& A_{5}=\gamma_{14}^{2} F_{4}+\gamma_{1} \gamma_{2} F_{5}+\gamma_{2}^{2} F_{6}+\gamma_{1} F_{7}+\gamma_{2} F_{8}+F_{9} \\
& A_{4}=\gamma_{110}^{2} F_{10}+\gamma_{12} \gamma_{11} F_{1}+\gamma_{2}^{2} F_{12}+\gamma_{1} F_{13}+\gamma_{2} F_{14}+F_{15} \\
& A_{3}=\gamma_{1}^{2} F_{16}+\gamma_{12} \gamma_{2} F_{17}+\gamma_{2}^{2} F_{18}+\gamma_{1} F_{19}+\gamma_{2} F_{20}+F_{21} \\
& A_{2}=\gamma_{122}^{2} F_{12}+\gamma_{12} \gamma_{23} F_{23}+\gamma_{24}^{2} F_{24}+\gamma_{125} F_{25}+\gamma_{226} F_{27}+F_{27} \\
& A_{1}=\gamma_{128}^{2} F_{28}+\gamma_{1} \gamma_{2} F_{29}+\gamma_{2}^{2} F_{30}+\gamma_{1} F_{31}+\gamma_{2} F_{32}+F_{33} \\
& A_{0}=\gamma_{134}^{2} F_{132}+\gamma_{15} \gamma_{2} F_{2}+\gamma_{26}^{2} F_{16} .
\end{aligned}
$$

The $F_{i}$ are functions of the system parameters and are defined in Appendix C.

\section{The Parametric Stability Analysis}

In this section, regions of stability, instability, and conditional stability will be plotted in the parameter space $\left(\gamma_{1}, \gamma_{2}\right)$ for the thermionic reactor model. The procedure will demonstrate the methods of mapping stability regions discussed in Chapter 3. 
The materials selected for the thermionic diode as well as the operating conditions are based on the models used by Guppy (1970a) and Gronroos (1967). The values of the parameters selected are given in Table 4.1. The number of radiator pipes and the radiator emissivity were chosen to make the radiator temperature above that which would normally be expected. This was done because this condition permitted the analysis of a stability zone containing two critical time delays. For this particular set of parameters, the $C_{i}$ and the $F_{i}$ take on values as shown in Table 4.2 .

Déscartes' Rule of Signs

In applying Déscartes' rule of signs to Eq. (4-44), we plot the curves $A_{i}=0$ in the space $\left(\gamma_{1}, \gamma_{2}\right)$. This procedure divides the space into regions in which the coefficients of Eq. (4-44) will have the same number of sign changes. We see from Eq. (4-45) that each curve $A_{i}=0$ is a conic; and therefore, by rotation and translation of the origin, it is possible to put these conics in standard form (Smail 1953). Using the values of $F_{i}$ given in Table 4.2, the rotation and translation (in that order) necessary to transform the conics into standard form are given in Table 4.3. Intercepts on the new axes and the signs of the coefficients in the neighborhood of the origin are also given. The range of interest in the space $\left(\gamma_{1}, \gamma_{2}\right)$ is roughly within the bounds

$$
\begin{array}{lc}
-0.02 \leqslant \gamma_{1} \leqslant 0.02 & \delta \mathrm{k} / \mathrm{k} / \mathrm{sec} \\
-0.01 \leqslant \gamma_{2} \leqslant 0.30 & \delta \mathrm{k} / \mathrm{k} / \mathrm{sec} .
\end{array}
$$


TABLE 4.1

Equilibrium Conditions and System Parameters

\begin{tabular}{|c|c|c|c|c|c|c|c|c|}
\hline \multicolumn{9}{|c|}{ System Material Parameters } \\
\hline I tem & Unit & $\begin{array}{l}\text { Fuel } \\
\text { (f) UC }\end{array}$ & $\begin{array}{l}\text { Emitter } \\
\text { (e) } \mathrm{W}\end{array}$ & $\begin{array}{l}\text { Collector } \\
\text { (c) Mo }\end{array}$ & $\begin{array}{l}\text { Insulator } \\
\text { (I) } \mathrm{Al}_{2} \mathrm{O}_{3}\end{array}$ & $\begin{array}{l}\text { Cladding } \\
\text { (c1) Nb }\end{array}$ & $\begin{array}{l}\text { Coolant } \\
\text { (co) Li-7 }\end{array}$ & $\begin{array}{l}\text { Radiator } \\
\text { (RP) SS }\end{array}$ \\
\hline$\rho_{i}$ & $\mathrm{gm} / \mathrm{cm}^{3}$ & 9.9 & 18.0 & 10.04 & 3.2 & 8.85 & 0.44 & 8.35 \\
\hline$C^{+}$ & $\mathrm{W}-\mathrm{s} / \mathrm{gm}-{ }^{\circ} \mathrm{K}$ & 0.268 & 0.188 & 0.294 & 1.19 & 0.31 & 4.14 & 0.837 \\
\hline $\mathrm{k}_{\mathrm{i}}$ & $\mathrm{W} / \mathrm{cm}^{\circ}{ }^{\circ} \mathrm{K}$ & 0.23 & 1.59 & 1.465 & 0.0347 & 0.63 & - & - \\
\hline $\mathrm{R}_{\mathbf{i}}$ & $\mathrm{cm}$ & 1.5 & 1.6 & 1.725 & $1: 775$ & 1.85 & - & 0.9 \\
\hline $\mathrm{L}_{i}$ & $\mathrm{~cm}$ & 5.0 & 5.0 & 5.0 & 6.0 & 6.0 & 6.0 & 250 \\
\hline$v_{i}$ & $\mathrm{~cm}^{3}$ & 35.4 & 4.87 & 5.26 & 2.75 & 5.13 & 8.78 & 314 \\
\hline
\end{tabular}

\begin{tabular}{|c|c|c|c|c|c|c|c|c|c|c|c|}
\hline \multicolumn{6}{|c|}{ Equilibrium Temperatures and Other Parameters } & \multicolumn{6}{|c|}{ Other Parameters } \\
\hline Item & Unit & Value & Item & Unit & Value & I tem & Unit & Value & Item & Unit & Value \\
\hline & ${ }^{\circ} \mathrm{K}$ & 2006 & $\lambda$ & $\sec ^{-1}$ & 0.1 & $n_{e}$ & - & 0.12 & $\mathrm{R}_{\text {PIN }}$ & $\mathrm{cm}$ & 0.9 \\
\hline $\mathrm{T}_{20}^{10}$ & ${ }^{\circ} \mathrm{K}$ & 1094 & $\beta$ & - & 0.0064 & $\mathrm{~N}_{\mathrm{DS}}$ & - & 5 & $\mathrm{U}_{\mathrm{coc}}$ & $\mathrm{cm} / \mathrm{sec}$ & 117 \\
\hline $\mathrm{T}_{\cos 0}^{20}$ & ${ }^{\circ} \mathrm{K}$ & 1044 & $\mathrm{v}_{\text {coc }}$ & $\mathrm{cm}^{3}$ & 8.78 & $\mathrm{~N}_{\mathrm{DP}}$ & - & 30 & $\mathrm{U}_{\mathrm{COR}}$ & $\mathrm{cm} / \mathrm{sec}$ & 40.4 \\
\hline $\mathrm{T}_{\text {coe } 0}$ & ${ }^{\circ} \mathrm{K}$ & 1069 & $\mathrm{v}_{\mathrm{coR}}$ & $\mathrm{cm}^{3}$ & 636.2 & $\mathrm{~N}_{\mathrm{RP}}$ & - & 50 & $\mathrm{~A}_{\mathrm{RC}}$ & $\mathrm{cm}^{2}$ & 2.55 \\
\hline $\mathrm{T}_{\mathrm{RO}}$ & ${ }^{\circ} \mathrm{K}$ & 1044 & $v_{R P}$ & $\mathrm{~cm}^{3}$ & 314 & $\omega_{\text {coc }}$ & $\mathrm{gm} / \mathrm{sec}$ & 75.3 & $\mathrm{H}$ & $\mathrm{W} / \mathrm{cm}^{2}{ }^{\circ} \mathrm{K}$ & 0.125 \\
\hline $\mathrm{T}_{\mathrm{Re}}$ & ${ }^{\circ} \mathrm{K}$ & 1019 & $\mathrm{~h}$ & $\mathrm{~W} / \mathrm{cm}^{2}-{ }^{\circ} \mathrm{K}$ & 6.0 & $\varepsilon_{r}$ & - & 0.8 & & & \\
\hline$n_{0}$ & $\mathrm{~W} / \mathrm{cm}^{3}$ & 100 & $\mathrm{~A}_{\mathrm{RS}}$ & $\mathrm{cm}^{2}$ & 1728 & $t_{\text {arm }}$ & $\mathrm{cm}$ & 0.2 & & & \\
\hline
\end{tabular}


TABLE 4.2

Values of $\mathrm{C}_{i}$ and $\mathrm{F}_{i}$ For Example

\begin{tabular}{|c|c|c|c|c|c|}
\hline$i$ & $\mathrm{C}_{i}$ & $F_{i}$ & $i$ & $c_{i}$ & $F_{i}$ \\
\hline 1 & 1. $322 \mathrm{E}+01$ & $-8.590 E-02$ & 19 & $3.516 \mathrm{E}+01$ & $-7.580 E+02$ \\
\hline 2 & 1. $316 \mathrm{E}+01$ & $-2.738 \mathrm{E}-01$ & 20 & $4.246 \mathrm{E}+00$ & $-1.768 \mathrm{E}+02$ \\
\hline 3 & $1.187 E+01$ & $2.104 \mathrm{E}+02$ & 21 & $2.584 \mathrm{E}+00$ & $2.648 E+02$ \\
\hline 4 & $1.948 \mathrm{E}-01$ & $2.104 \mathrm{E}+02$ & 22 & $3.581 E+00$ & $3.243 E+02$ \\
\hline 5 & $2.268 \mathrm{E}+00$ & $4.171 \mathrm{E}-01$ & 23 & $4.634 \mathrm{E}-01$ & $8.600 E+01$ \\
\hline 6 & $8.019 \mathrm{E}-01$ & $1.875 \mathrm{E}-02$ & 24 & $7.195 \mathrm{E}-02$ & $5.772 \mathrm{E}+00$ \\
\hline 7 & $8.030 E+00$ & $-1.807 E+01$ & 25 & $1.261 E-01$ & $-2.028 E+01$ \\
\hline 8 & $1.836 \mathrm{E}+00$ & $-5.539 E+01$ & 26 & $1.693 E-02$ & $-3.476 E+00$ \\
\hline 9 & $1.062 \mathrm{E}-01$ & $9.239 \mathrm{E}+03$ & 27 & $-3.466 E-01$ & $1.274 E+00$ \\
\hline 10 & $2.125 E+01$ & $9.241 E+03$ & 28 & 3. $373 \mathrm{E}-01$ & $2.867 E+00$ \\
\hline 11 & $2.130 E+01$ & $6.322 \mathrm{E}+01$ & 29 & $-1.061 E+00$ & $9.580 \mathrm{E}-01$ \\
\hline 12 & $1.206 \mathrm{E}+02$ & $3.681 \mathrm{E}+00$ & 30 & $-1.076 \mathrm{E}+00$ & $6.868 \mathrm{E}-02$ \\
\hline 13 & $1.215 \mathrm{E}+02$ & $-7.936 \mathrm{E}+02$ & 31 & $-4.745 E-02$ & $-4.347 \mathrm{E}-02$ \\
\hline 14 & $2.707 E+00$ & $-2.358 \mathrm{E}+03$ & 32 & $-5.941 E-02$ & $-1.640 \mathrm{E}-02$ \\
\hline 15 & $1.264 \mathrm{E}+02$ & $.8 .853 \mathrm{E}+03$ & 33 & $-1.041 \mathrm{E}-01$ & $1.647 \mathrm{E}-03$ \\
\hline 16 & 1. $316 \mathrm{E}+02$ & $8.916 \mathrm{E}+03$ & 34 & $-4.745 E-03$ & $5.060 \mathrm{E}-03$ \\
\hline 17 & $1.332 \mathrm{E}+01$ & $2.304 E+03$ & 35 & & $3.282 E-03$ \\
\hline 18 & $3.002 E+01$ & $1.546 \mathrm{E}+02$ & 36 & & $2.642 \mathrm{E}-04$ \\
\hline
\end{tabular}


Therefore, from Table 4.3, it is obvious that, within this range, only $A_{1}$ and $A_{0}$ exhibit variations of sign. The remaining coefficients are positive. Figure 4.5 shows the curves $A_{0}=0$ and $A_{1}=0$ in the parameter space $\left(\gamma_{1}, \gamma_{2}\right)$. The number of variations in sign, (C) is shown in each zone.

TABLE 4.3

Standard Forms of $\mathrm{A}_{i}$

\begin{tabular}{|c|c|c|c|c|c|c|c|}
\hline \multirow[b]{2}{*}{ coef. } & \multirow{2}{*}{$\begin{array}{l}\text { rotat. } \\
\text { (deg.) }\end{array}$} & \multicolumn{2}{|c|}{ new origin } & \multicolumn{2}{|c|}{ intercepts } & \multirow{2}{*}{$\begin{array}{l}\text { type of } \\
\text { conic }\end{array}$} & \multirow{2}{*}{$\begin{array}{l}\text { behavior } \\
\text { near origin }\end{array}$} \\
\hline & & $\bar{x}$ & $y$ & $\bar{x}$ & $\mathrm{y}$ & & \\
\hline $\mathrm{A}_{6}$ & 0 & 0.043 & 772 & 0 & 0 & $\begin{array}{c}\text { upward opening } \\
\text { parabola }\end{array}$ & + \\
\hline$A_{5}$ & 0.057 & 0.043 & 1493 & 11.5 & 1200 & ellipse & + \\
\hline $\mathrm{A}_{4}$ & 0.20 & 0.043 & 330 & 6.40 & 326 & ellipse & + \\
\hline $\mathrm{A}_{3}$ & 7.37 & 0.043 & 6.84 & 0.045 & 1.82 & ellipse & + \\
\hline $\mathrm{A}_{2}$ & 7.55 & 0.031 & 5.58 & 0.061 & 4.07 & ellipse & + \\
\hline $\mathrm{A}_{1}$ & 9.45 & 0.008 & -0.41 & n.a. & 0.55 & hyperbola & var. \\
\hline $\mathrm{A}_{0}$ & 17.2 & 0 & 0 & 0 & 0 & $\begin{array}{l}\text { degenerate } \\
\text { hyperbola }\end{array}$ & var. \\
\hline
\end{tabular}

In our previous discussion, it was pointed out that, in regions where $C=1$, we know there exists one positive real root to Eq. (4-44). However, in the region where $\mathrm{C}=2$, we can not yet be sure whether there are indeed two roots or no roots. This region will be further resolved later. First, however, it is instructive to determine more information about the stability within the region where $C=1$. 


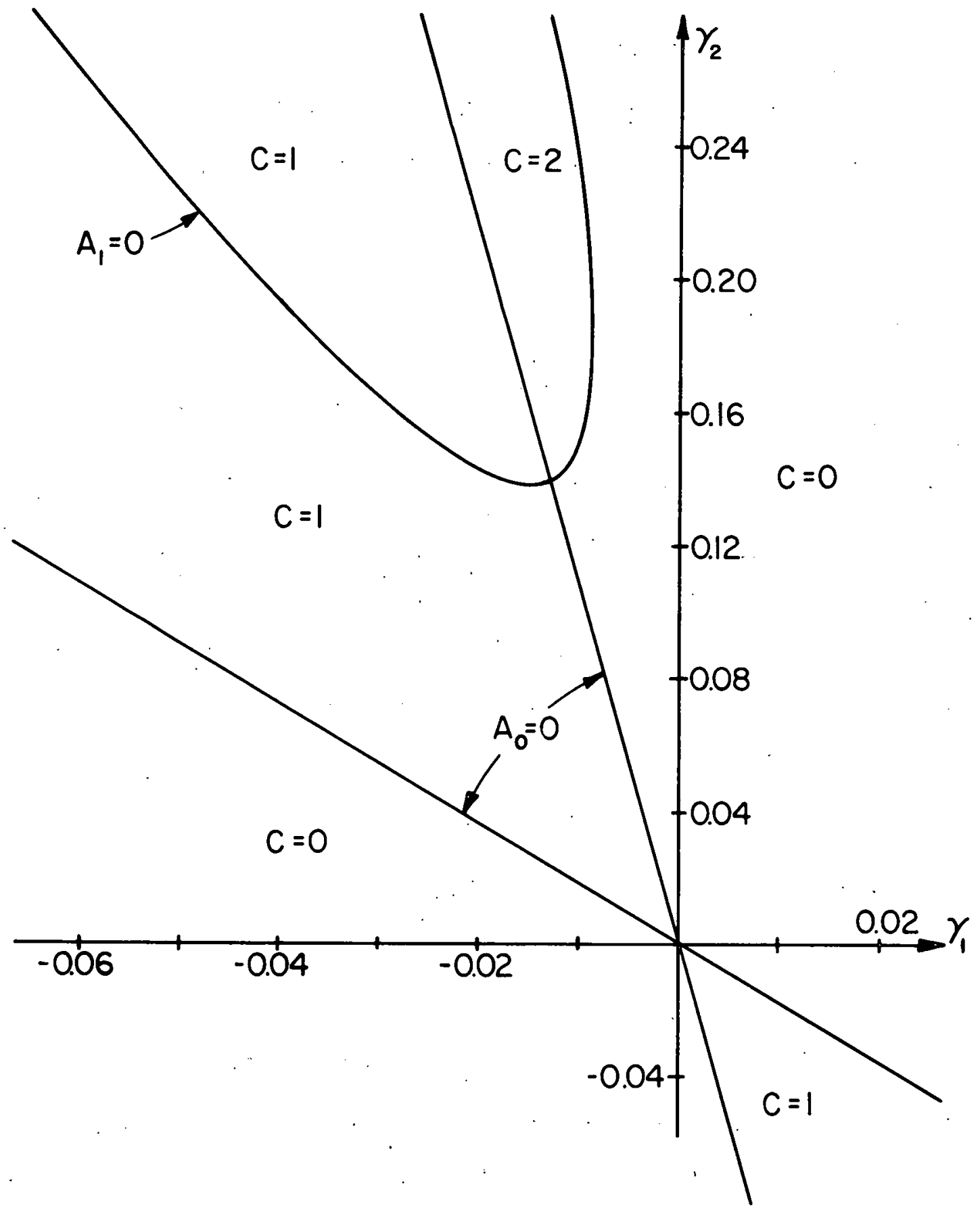

Figure 4.5. Results of Déscartes' Rule of Signs 
As was discussed in Chapter 2, El'sgol'ts (1966) has given a theorem which states that if a linear system is stable for $\tau=0$, then it is also stable for positive values of $\tau$ if they are small enough. Similar assertions can be made for systems which are unstable for $\tau=0$. Therefore, it should be possible to gain more information about system stability from a Routh-Hurwitz analysis of the characteristic equation given by Eq. (4-39) with $\tau=0$.

The Routh-Hurwitz analysis was carried out in the following manner. A computer program was written to multiply polynomials in two variables whïle keeping these variables distinct. With this program, the polynomials making up the first column of the Routh array were calculated. Zeros of these polynomials were then plotted in the physically interesting range of $\left(\gamma_{1}, \gamma_{2}\right)$ with the aid of a standard polynomial-root-solving numerical technique. From these computations, the stability boundaries shown in Fig. 4.6 were determined.

In Fig. 4.6, the straight line positioned at approximately $135^{\circ}$ and extending into the fourth quadrant is a plot of the condition

$$
\gamma_{1}\left(C_{25}+C_{33}\right)+\gamma_{2}\left(C_{26}+C_{34}\right)=0
$$

which results from the constant terms of $Q(s)$ and $R(s)$. Utilizing the definitions of $\mathrm{F}_{34}, \mathrm{~F}_{35}$, and $\mathrm{F}_{36}$ given in Appendix $\mathrm{C}$, we can write the equation $A_{0}=0$ as

$$
\begin{aligned}
\gamma_{1}^{2}\left(C_{25}^{2}-C_{33}^{2}\right)+2 \gamma_{12} \gamma_{2}\left(C_{25} C_{26}-C_{33} C_{34}\right)+ \\
+\gamma_{2}^{2}\left(C_{26}^{2}-C_{34}^{2}\right)=0 .
\end{aligned}
$$




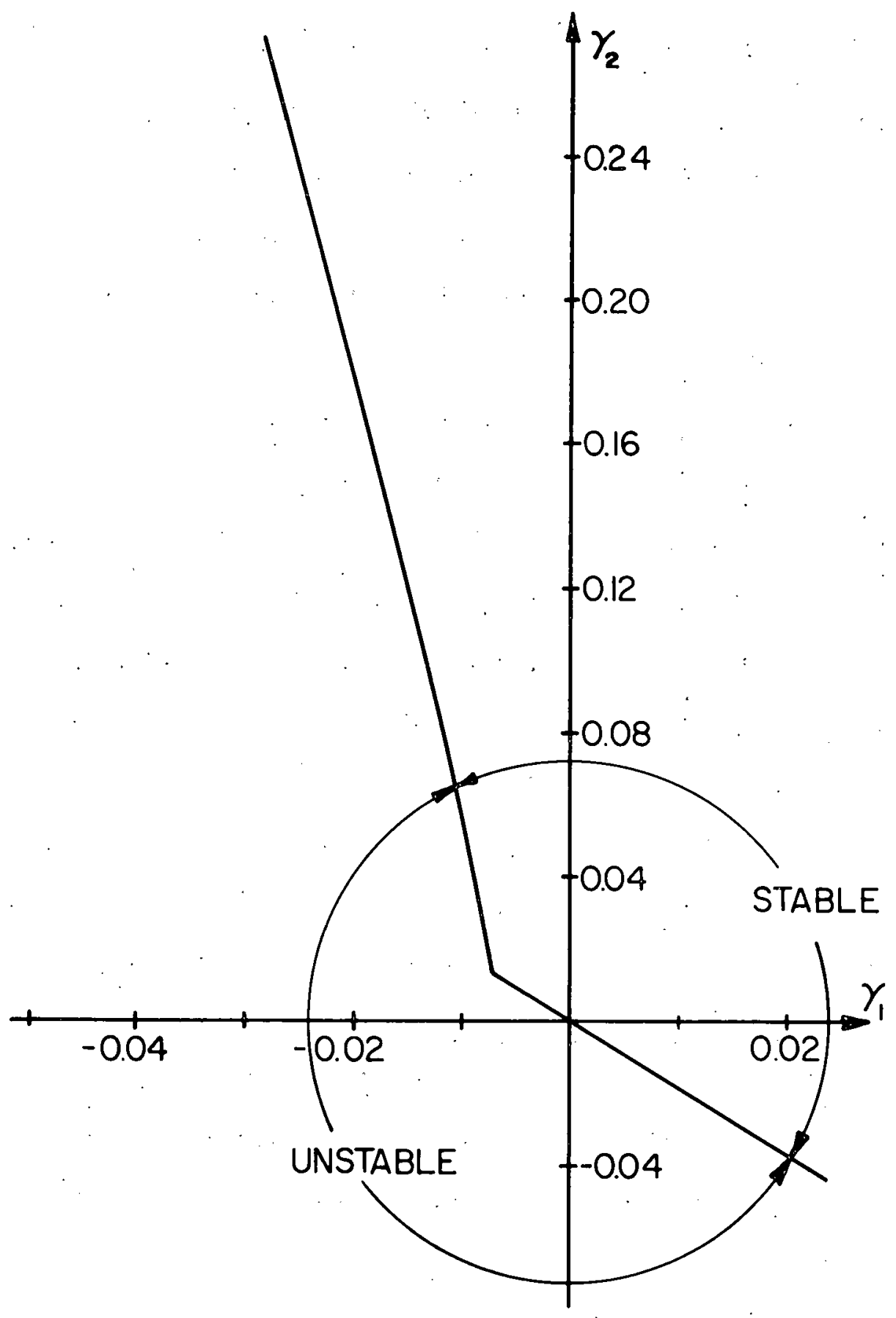

Figure 4.6. Zero-Delay Stability Results 
Equation (4-48) can be factored to give

$$
\begin{aligned}
{\left[\gamma_{1}\left(C_{25}+C_{33}\right)+\right.} & \left.\gamma_{2}\left(C_{26}+C_{34}\right)\right] x \\
& \times\left[\gamma_{1}\left(C_{25}-C_{33}\right)+\gamma_{2}\left(C_{26}-C_{34}\right)\right]=0 .
\end{aligned}
$$

Thus it is seen that the branch of the degenerate hyperbola given in the first set of brackets is identical to the Routh condition given in $\mathrm{Eq} \cdot(4-47)$

Therefore, if we superimpose the results of the zero-delay analysis with the results of the Déscartes' analysis, we obtain the stability zones shown in Fig. 4.7.

Sturm's Theorem

In thj.s section, we will use Sturm's theorem to resolve the as yet unknown region shown in Fig. 4.7. Unfortunately, in order to apply this theorem in a strict analytical form to the thermionic model, solutions of $124^{\text {th }}$ order polynomials would be necessary. Solutions of this order were not practicable in this work. Instead, a numerical search technique was used.

The search technique was written to consider the Sturm sequences $V(0)$ and $V(\infty)$ at each point of a fine grid covering the unknown region. From these sequences, the number of real roots to Eq. (4-44) at each point could be determined. Then, after the approximate boundaries had been indicated from the grid analysis, the boundaries were further delineated with a homing technique. A digital computer was used in performing these searches. 


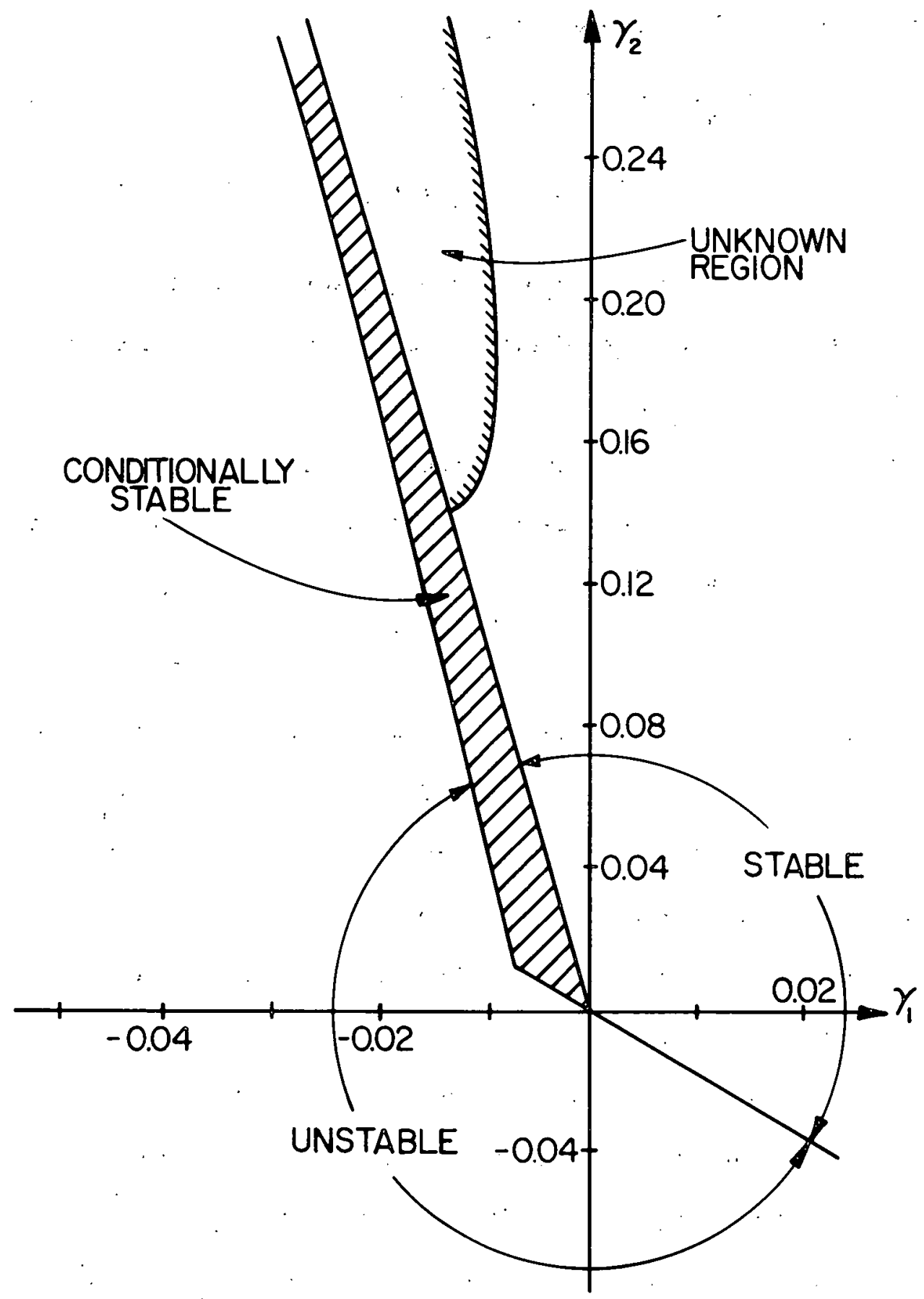

Figure 4.7. Superimposed Results of Déscartes and Zero-Delay Analysis 
The results of this analysis indicated that only one element of the Sturm sequences $V(0)$ and $V(\infty)$ was important in the unknown regions of Fig. 4.7. This element was the final constant entry ( $G_{1}$ ) identical in both the $V(0)$ and $V(\infty)$ sequences. A graph of $G_{1}=0$ is shown in Fig. 4.8. Although nearly all of the elements of $V(0)$ pass through zeros to the left of the degenerate hyperbola, these were left unresolved since they were unimportant in delineating zones with positive real roots. Hence, Fig. 4.8 has an unresolved area to the left of the degenerate hyperbola. In Fig. 4.8, the expansion of the abscissa relative to previous figures should be noted. The point denoted in this figure will be discussed in the next section.

The Stability Results

Superimposing the results of the Sturm sequence analysis with those presented in Fig. 4.7 gives the complete stability analysis results in the physical range of the parameters $\left(\gamma_{1}, \gamma_{2}\right)$. These results are shown in Fig. 4.9. We see that there are regions which are stable for any value of the time delay and regions which are unstable for any delay. In addition, there is a conditionally stable region which is stable for $\tau=0$. Within this region, there are two subregions: the first being stable within a time delay range $0 \leqslant \tau \leqslant \tau$ crit-f, and the second admitting the possibility of stability bands. It is instructive to calculate some of the critical values to indicate how stability depends on the delay within the conditionally stable regions. They are calculated by Eq. (3-37). 


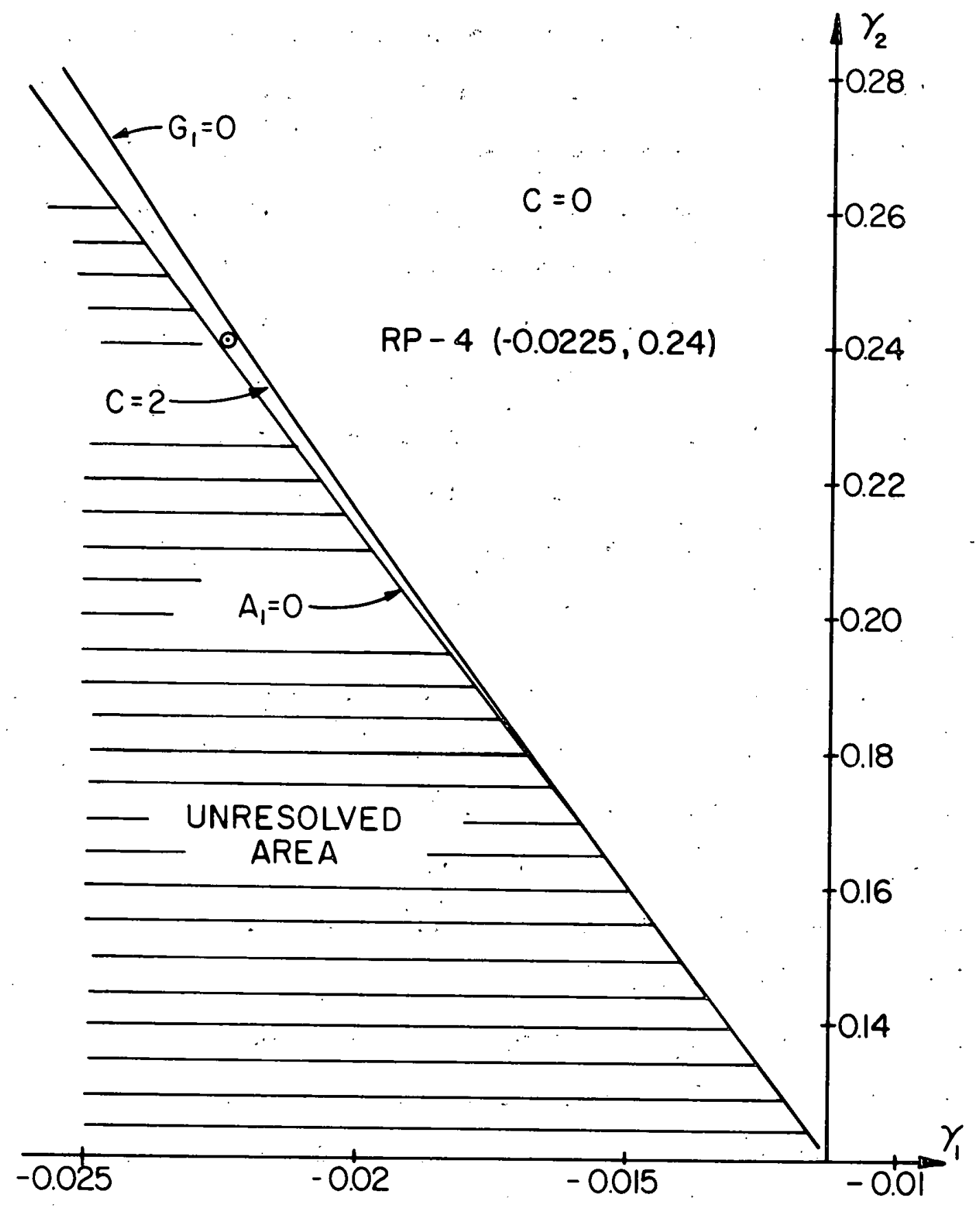

Figure 4.8. Results of Sturm's Sequence 


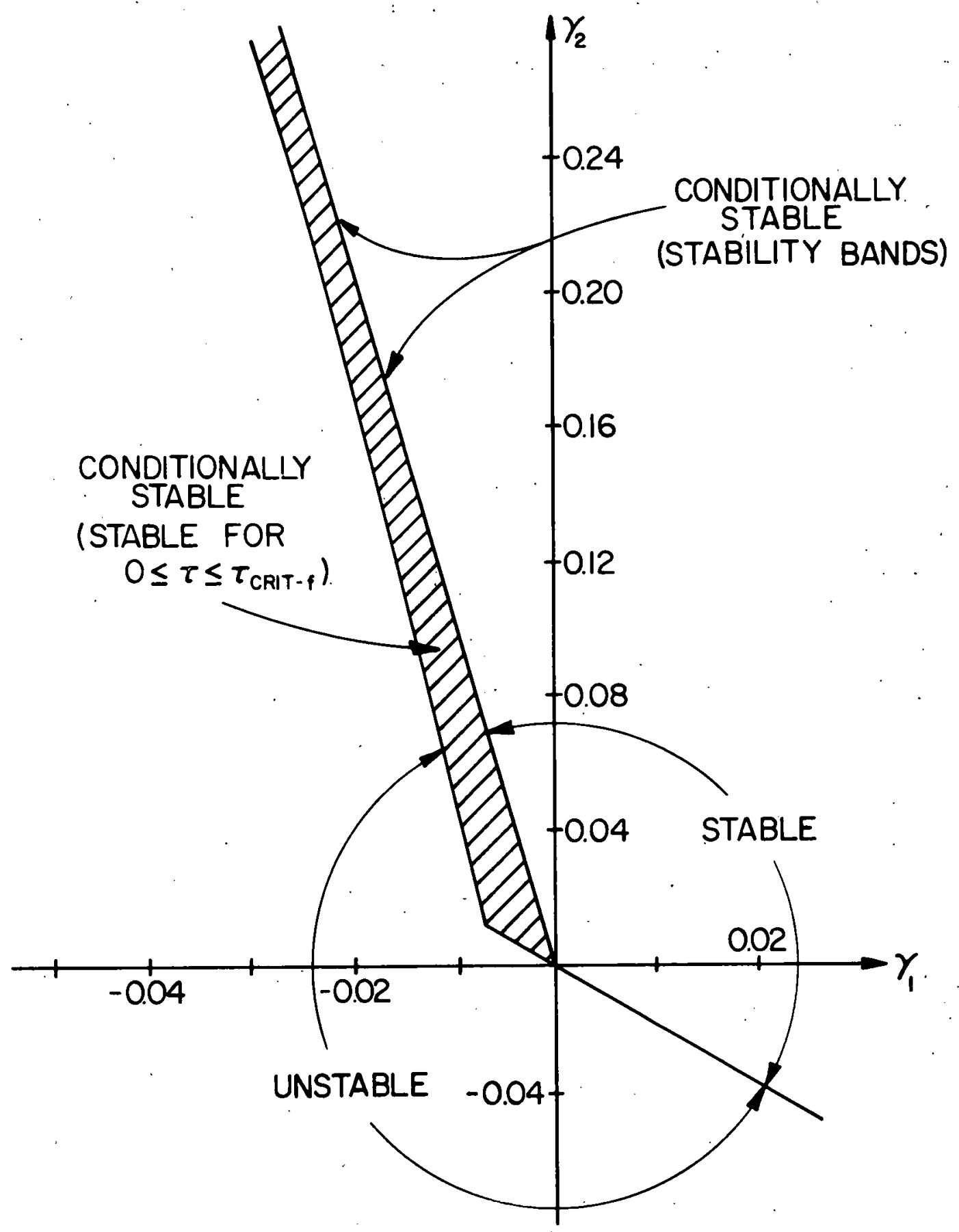

Figure 4.9. Regions of Stability for Thermionic Reactor Model 
Figure 4.10 again shows the stability analysis results with several reference points (RP) and reference lines (RL) indicated. With a standard root solving numerical technique, the following results were obtained for RP-1.

$$
\begin{aligned}
& \omega_{1}=1.385 \times 10^{-2} \sec ^{-1} \\
& \tau_{10}=4.31 \mathrm{sec} \\
& \tau_{11}=458 \mathrm{sec} \\
& \tau_{12}=912 \mathrm{sec}
\end{aligned}
$$

Therefore, from our previous discussion, we know that the system is stable within the range

$$
0 \leqslant \tau \leqslant 4.31 \mathrm{sec}
$$

and unstable if $\tau>4.31 \mathrm{sec}$. That this is true can be further demonstrated by Modified Mikhailov curves for $\tau=1 \mathrm{sec}$. (Fig. 4.11 a), $\tau=10 \mathrm{sec} .($ Fig. $4.11 \mathrm{~b}$ ), and $\tau=550 \mathrm{sec}$ (Fig. 4.12). In these figures, we see that increasing the time delay tends to open the spiral of the Modified Mikhailov curve. A critical time delay is indicated when a loop of the spiral changes its position with respect to the origin. At $\tau=4.31$ seconds, the first loop will pass to the opposite side of the origin, and the system passes from stability to instability. At $\tau=458$ seconds, a second loop passes to the opposite side of the origin. This, however, has no effect on the system stability since the system remains unstable.

For comparison; Modified Mikhailov curves are plotted at RP-2 (Fig. 4.13) for $\tau=1$ second and at $R P-3$ (Fig. 4.14) for $\tau=10$ seconds. It is seen that these curves are very similar to their counterparts in 


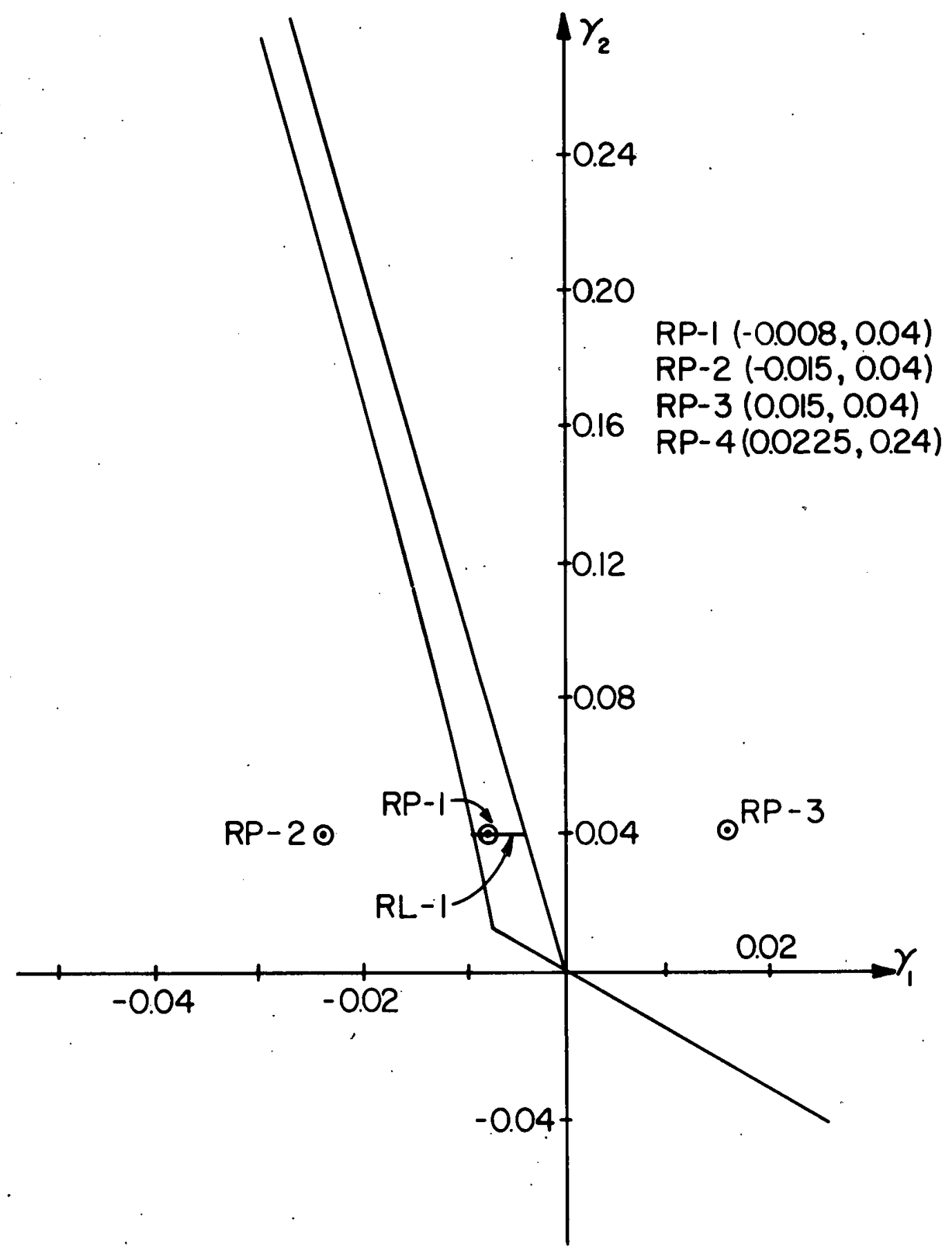

Figure 4.10. Locations of Reference Points and Reference Lines 


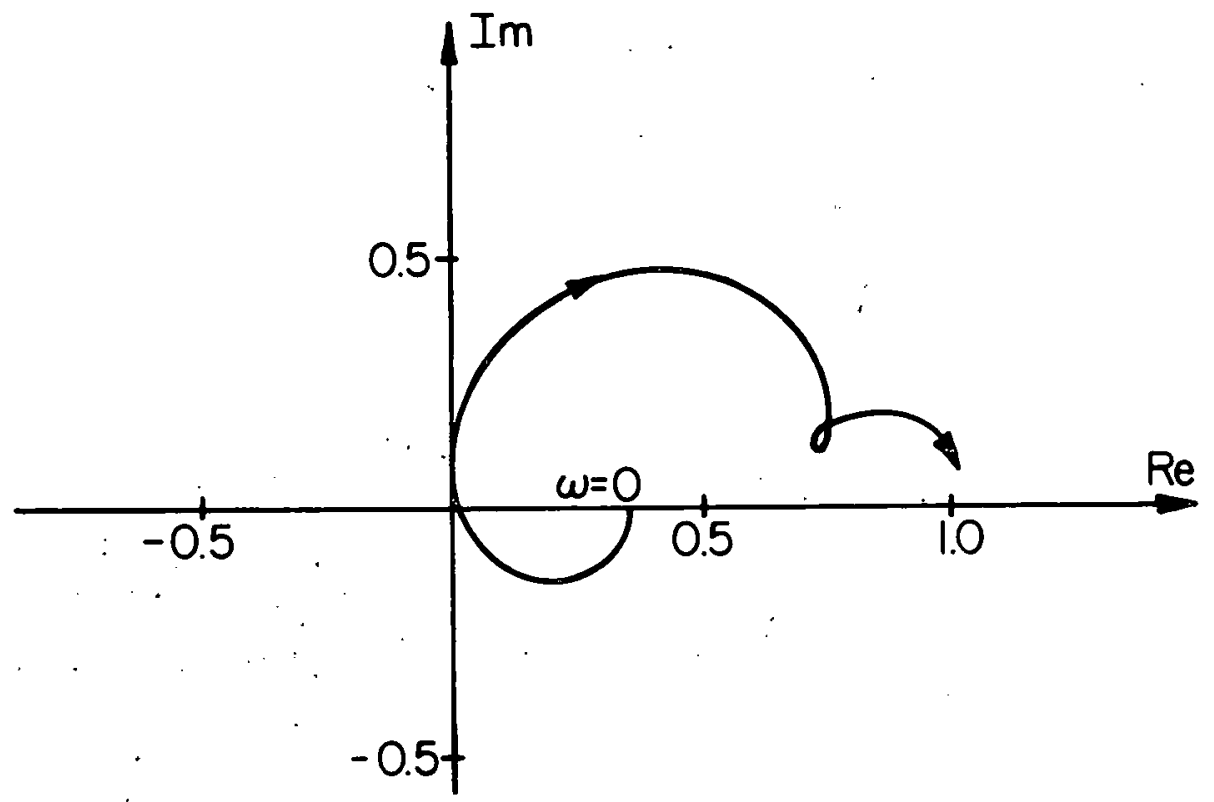

a. $\tau=1 \mathrm{sec}$

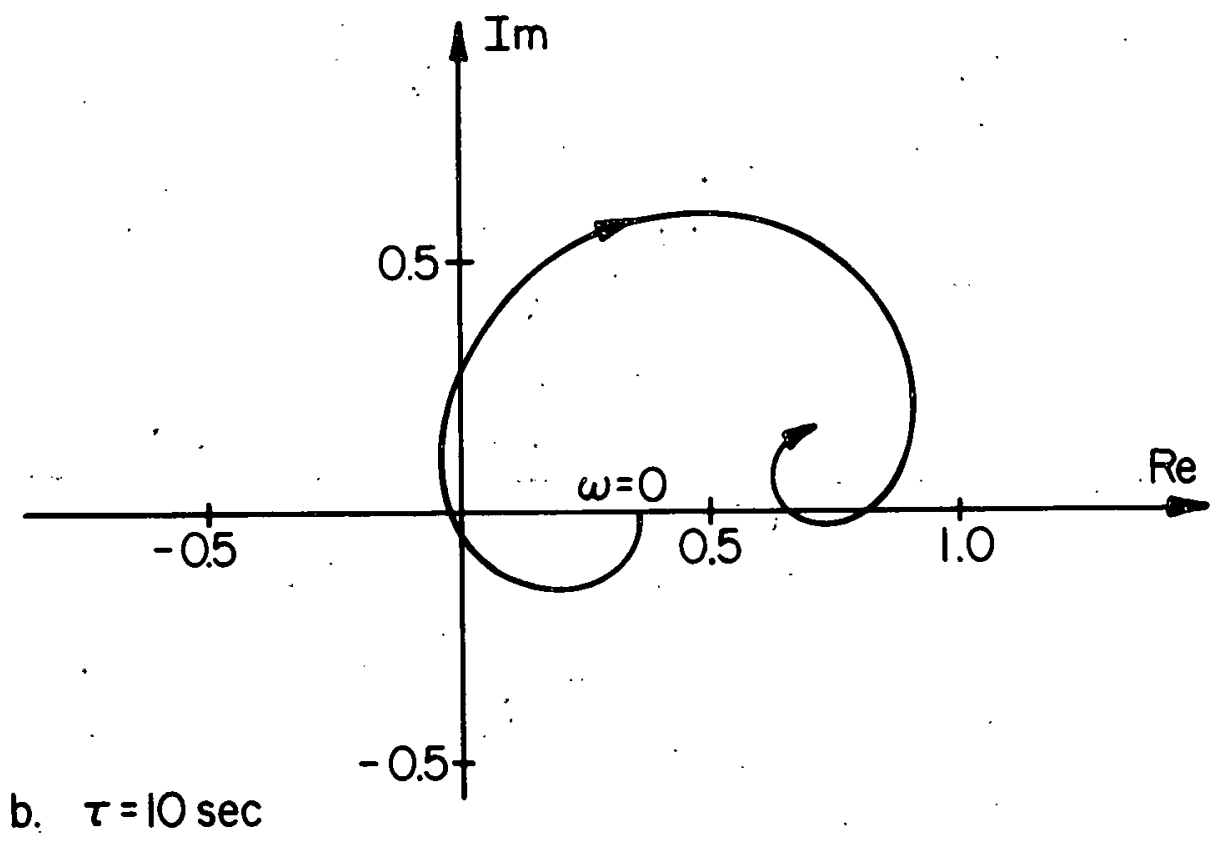

Figure 4,1i. Modified Mikhailov Curves at RP-1. $\tau=1 \mathrm{sec} . \tau=10 \mathrm{sec}$. 


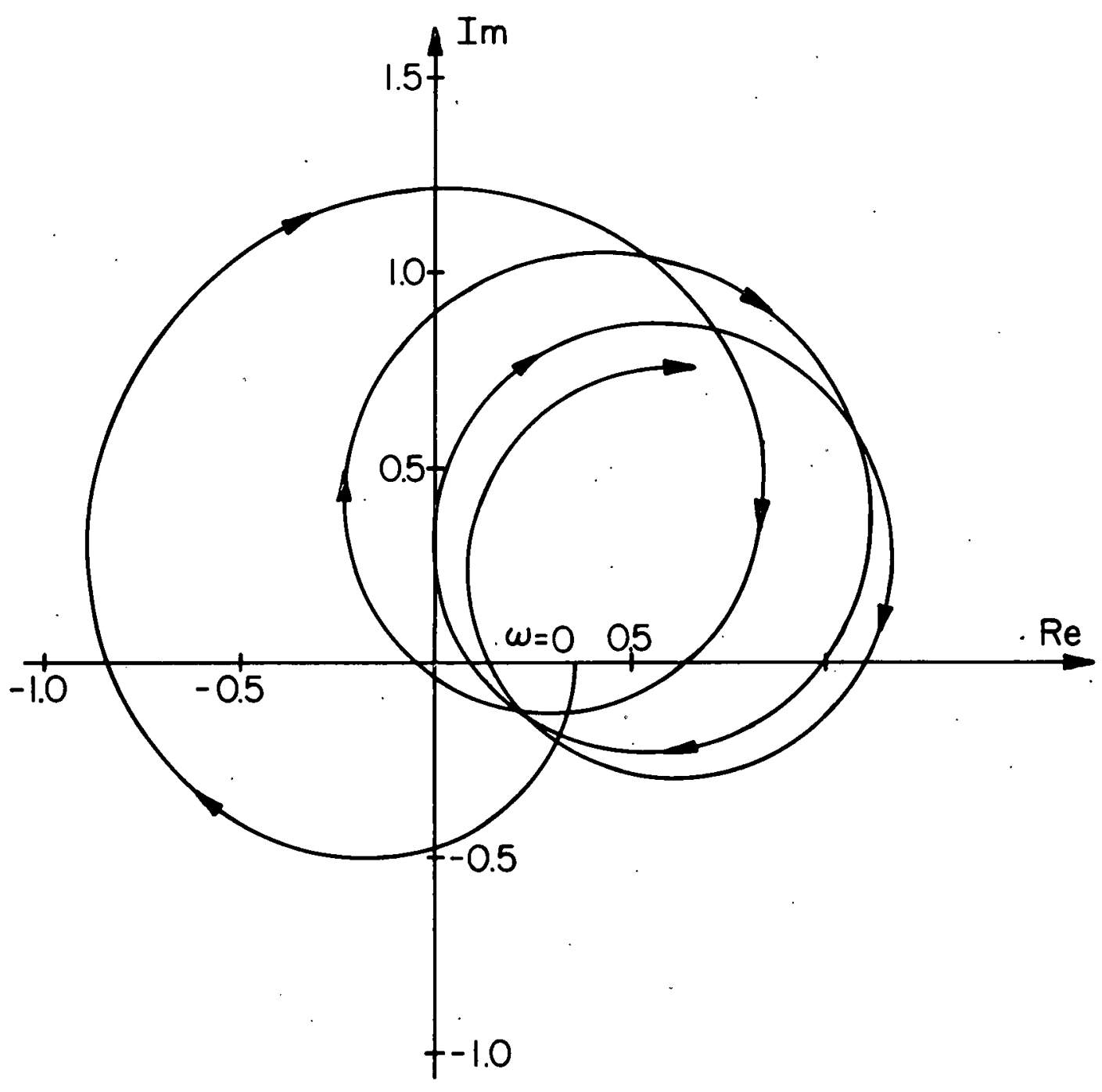

Figure 4.12. Modified Mikhailov Curve at RP-1. $\tau=550 \mathrm{sec}$. 


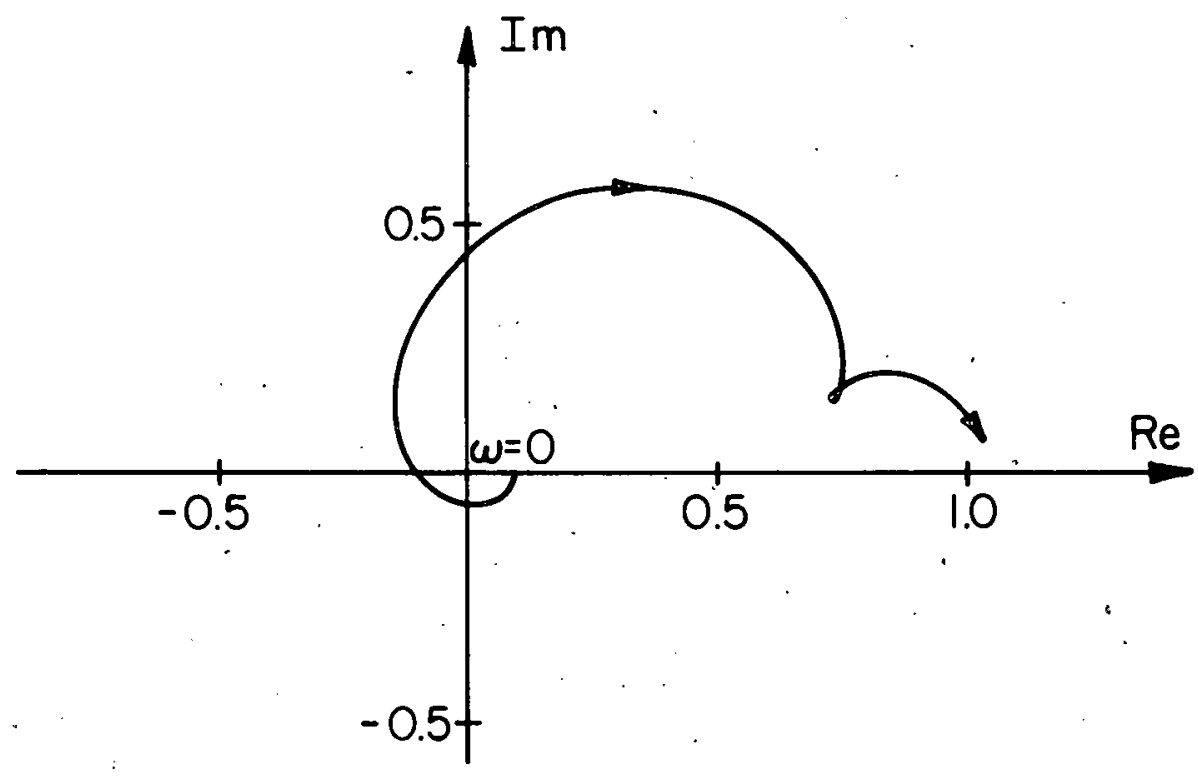

Figure 4:13. Modified Mikhailov Curve at RP-2. $\tau=1 \mathrm{sec}$.

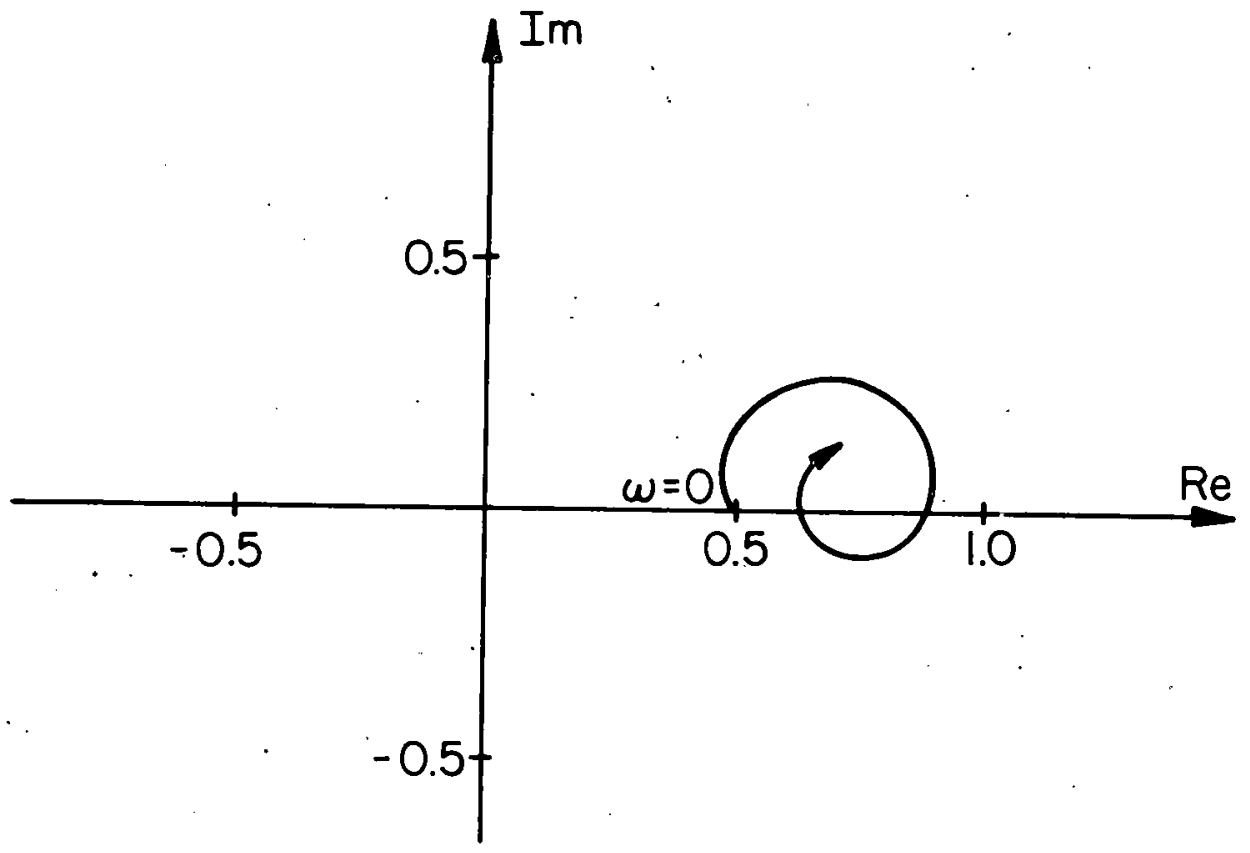

Figure 4.14. Modified Mikhailov Curve at RP-3. $\tau=10 \mathrm{sec}$. 
Figs. 4.11 and 4.12 except. that the curves have been translated somewhat increasing the stability margin in the case of $\mathrm{RP}-3$, but decreasing it for $\mathrm{RP}-2$.

It is interesting to, plot $\tau_{\text {crit-f }}$ as a function of $\gamma_{1}$ along RL-1. This plot is shown in Fig. 4.15. As the value of $\gamma_{1}$ moves closer to the stable first quadrant, the stable range of delays $(0 \leqslant \tau \leqslant \tau$ crit-f $)$ grows rapidly larger. This supports the intuitive notion that the system becomes less stable as the stability boundary is approached.

Consider the physical processes which might result from a small positive perturbation in the neutron power density at RP-1. Initially, there would be a positive reactivity feedback resulting from the positive Doppler coefficient in the fuel region. In a very short time, however, enough of the heat produced from the perturbation would be conducted to the collector-insulator-clad region to produce a net negative reactivity feedback. Until $\tau$ seconds had elapsed, the coolant inlet temperature would remain constant, and the system would behave as the system under the infinite-delay approximation. (In the next section, we will see that the infinite-delay approximation is unstable at $\mathrm{RP}-1$ ). The system would then decrease its power level to a point below the equilibrium at which time the reactivity feedback would again be positive. The system would continue in this manner, and unstable oscillations would result. If, however, an increase in the coolant inlet temperature occurred at a time when the power was decreasing, less heat than before would be conducted away from region two thus tending to negate the positive reactivity due to the decrease of the temperature of this region below the equilibrium value. The coolant recirculation would 


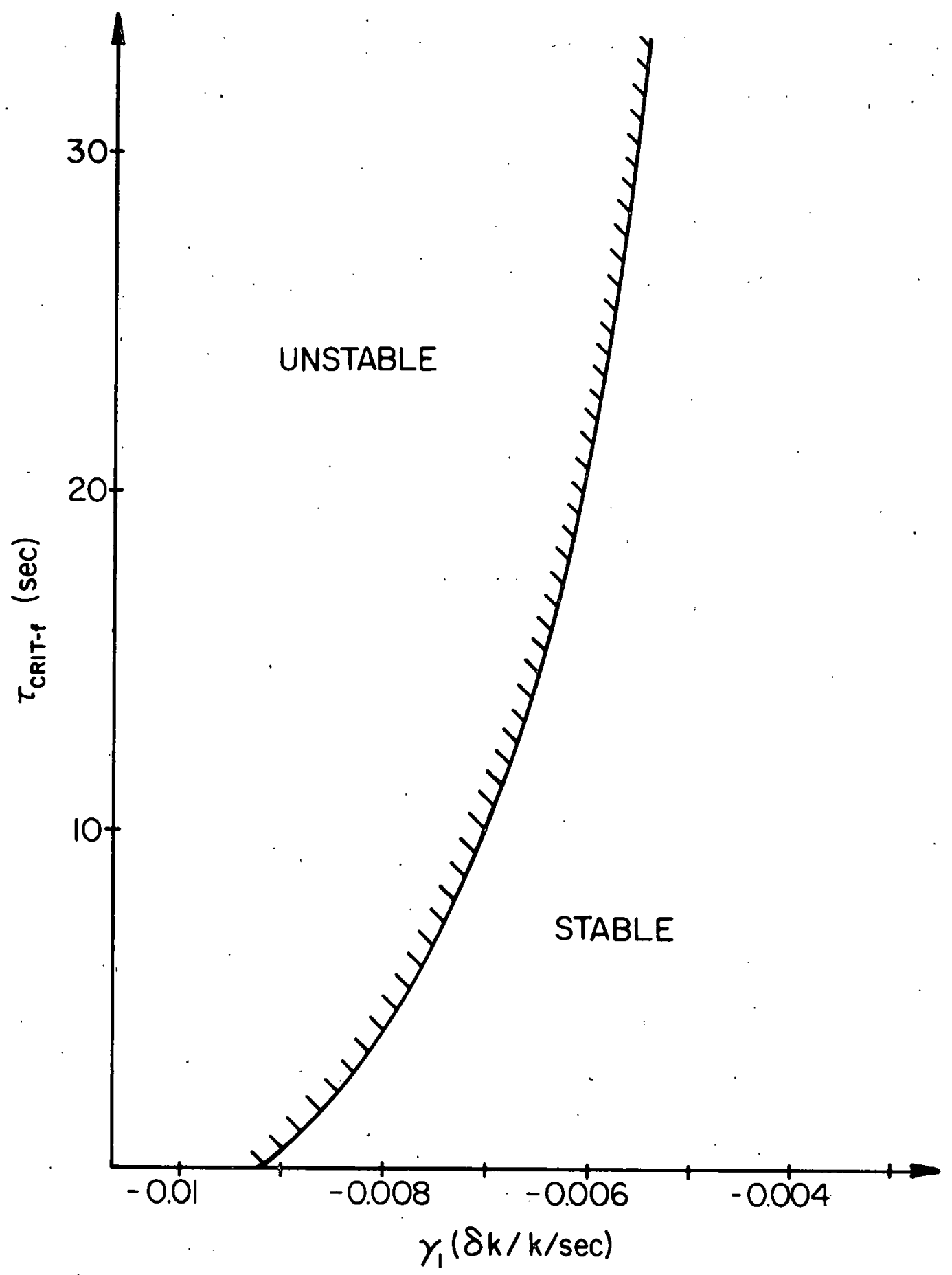

Figure 4.15. $\tau_{\text {crit-f }}$ versus $\gamma_{1}$ on $R L-1$ 
thus enhance the system stability. On the other hand, the coolant could be delayed in a manner which would detract from the system stability. With a standard root solving technique, the following results were obtained at $\mathrm{RP}-4$ which is shown in Fig. 4.8 .

$$
\begin{array}{llll}
\omega_{1} & =2.979 \times 10^{-2} & \sec ^{-1} & \omega_{2}=7.100 \times 10^{-3} \mathrm{sec}^{-1} \\
\tau_{10} & =17.5 & \text { sec } & \tau_{20}=323 \mathrm{sec} \\
\tau_{11}=228 & \text { sec } & \tau_{21}=1209 \mathrm{sec} \\
\tau_{12}=439 & \text { sec } &
\end{array}
$$

Therefore, from our previous discussion, we can determine the following stability conclusions.

$$
\begin{array}{cl}
0 \leqslant \tau \leqslant 17.5 & \text { Stable } \\
17 . \dot{5} \leqslant \tau \leqslant 228 & \text { Unstable } \\
228 \leqslant \tau \leqslant 323 & \text { Unstable } \\
\tau \geqslant 323 & \text { Unstable }
\end{array}
$$

For RP-4, Modified Mikhailov plots for $\tau=10$ seconds, $\tau=75$ seconds, $\tau=275$ seconds, and $\tau=375$ seconds are shown in Fig. 4.16a, 4.16b, 4.17, and 4.18 respectively. These figures confirm the conclusions of $\mathrm{Eq} \cdot(4-53)$.

In Fig. 4.16a, we see that there are no encirclements of the origin and that the system is stable. By increasing the delay through the critical value of 17.5 seconds to 75 seconds, Fig. $4.16 \mathrm{~b}$ shows that we have driven one loop of the spiral to the opposite side of the origin indicating the system is now unstable. A further increase in the delay through the critical value of 228 seconds drives one more loop to the opposite side of the origin (Fig. 4.17). Increasing the delay once 


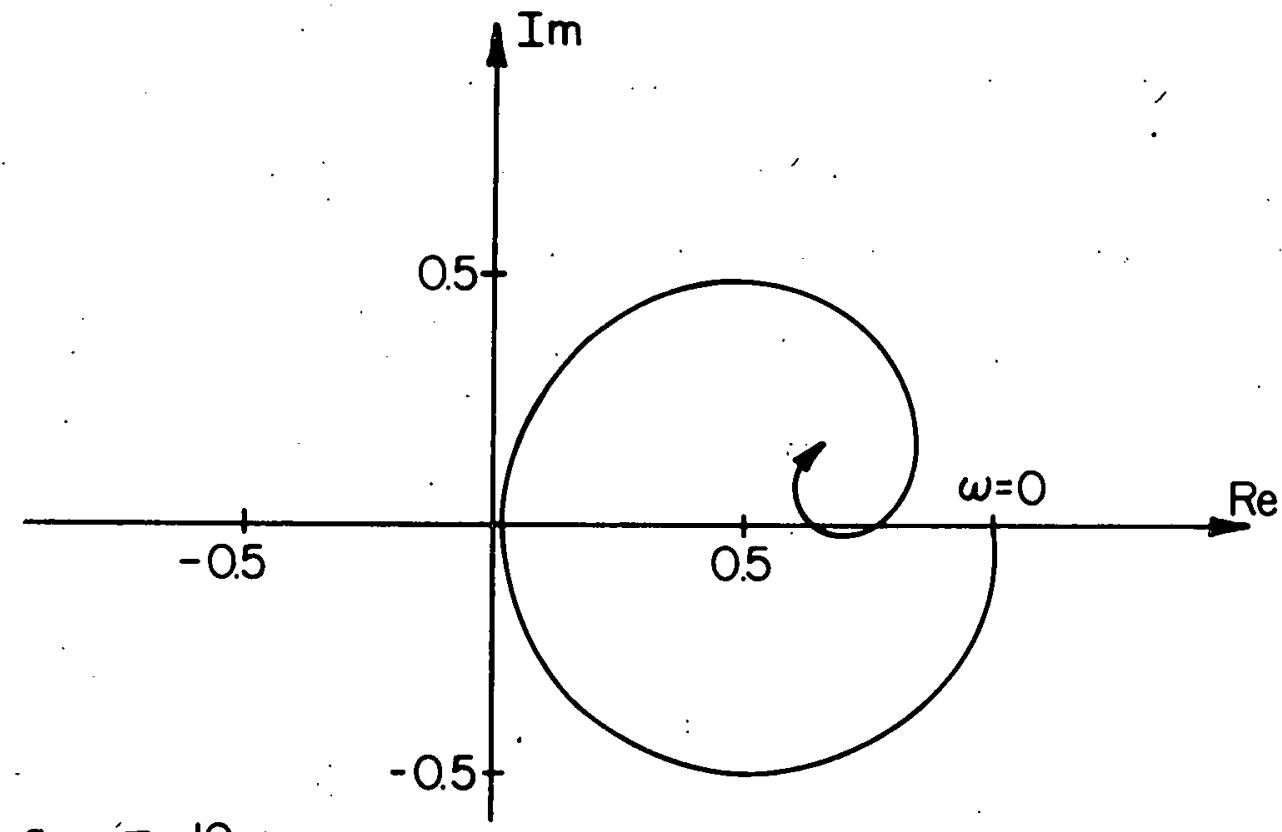

a. $\tau=10 \mathrm{sec}$

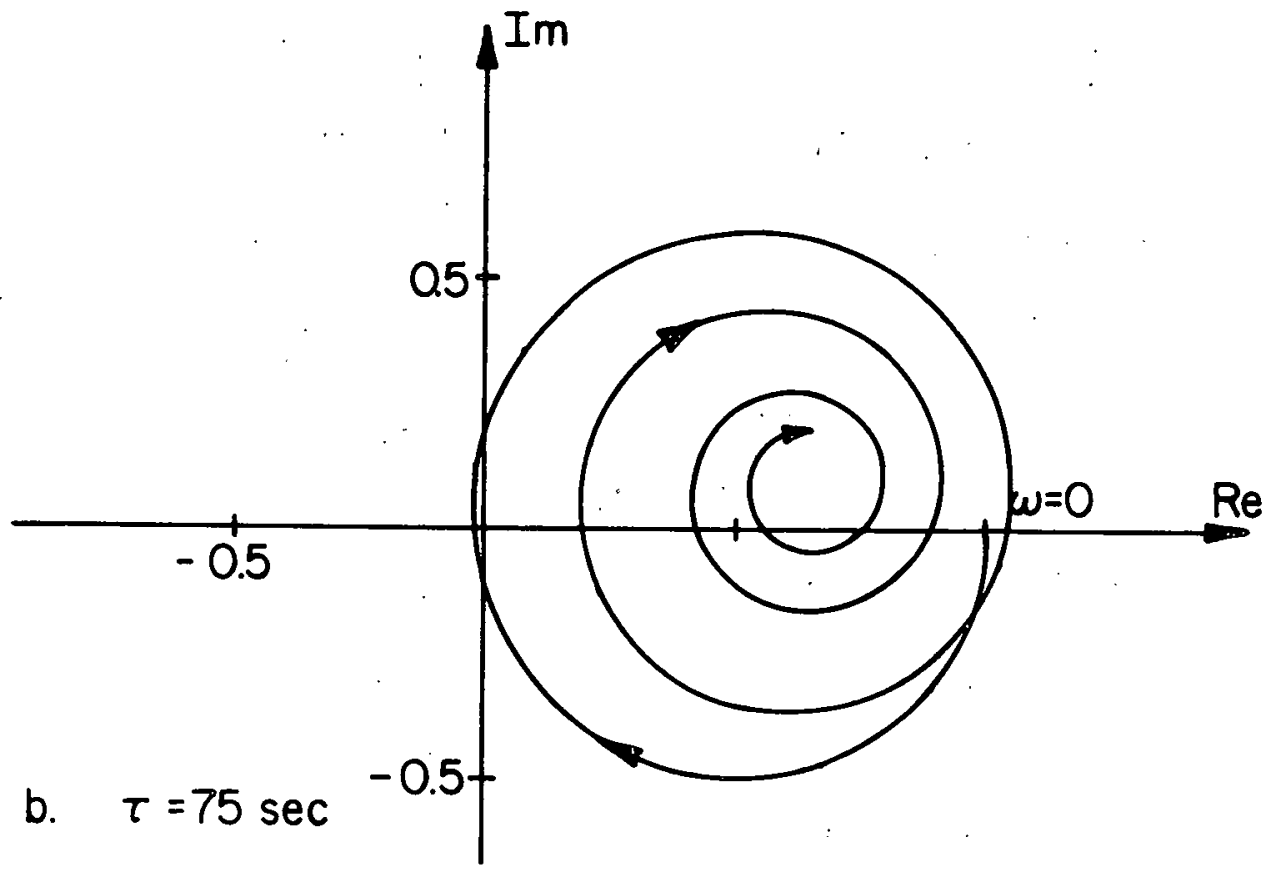

Figure 4.16. Modified Mikhailov Curves at RP-4:

$\tau=10 \mathrm{sec} . \quad \tau=75 \mathrm{sec}$. 


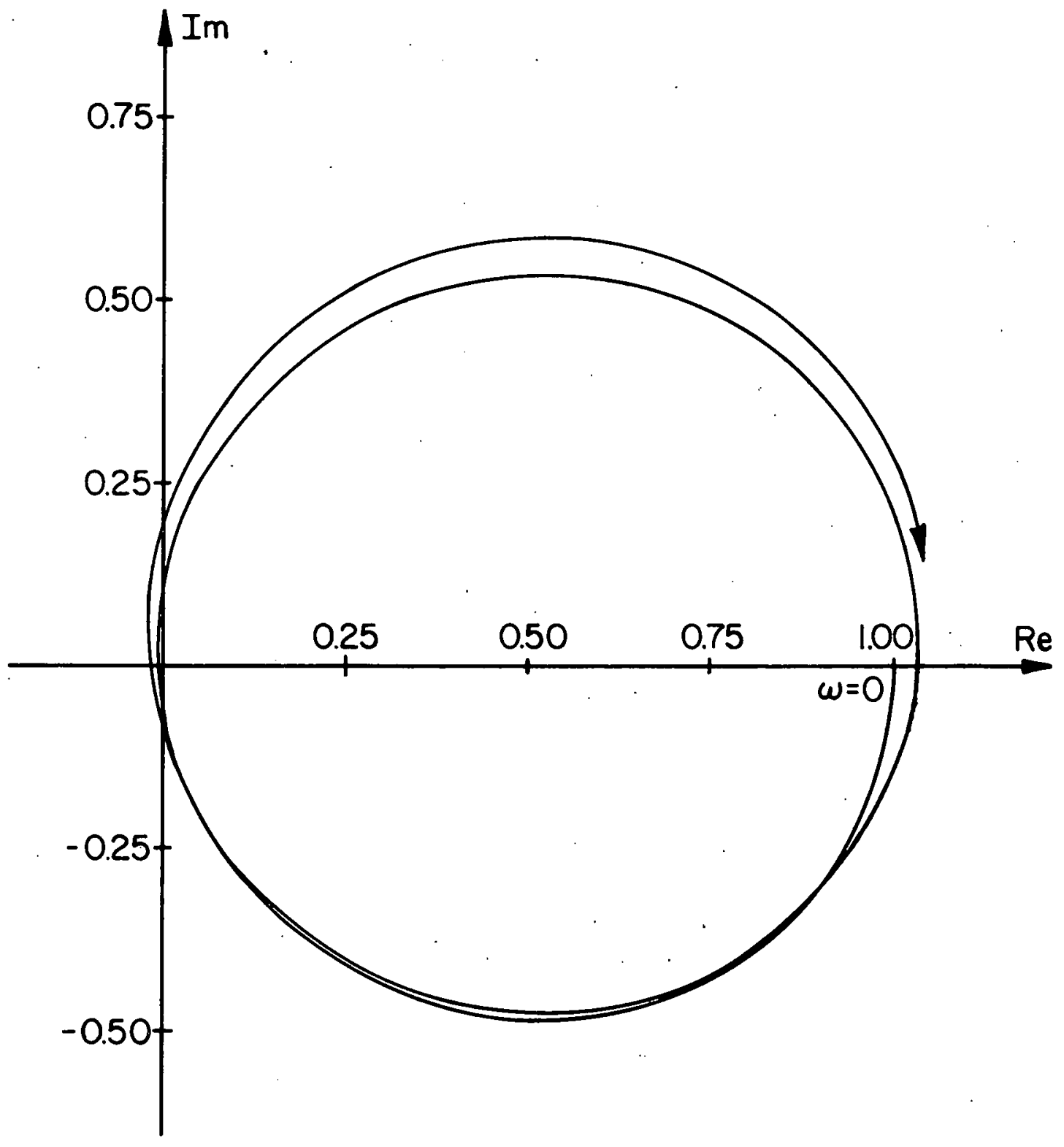

Figure 4.17. Modified Mikhailov Curve at RP-4. $\tau=275 \mathrm{sec}$. 


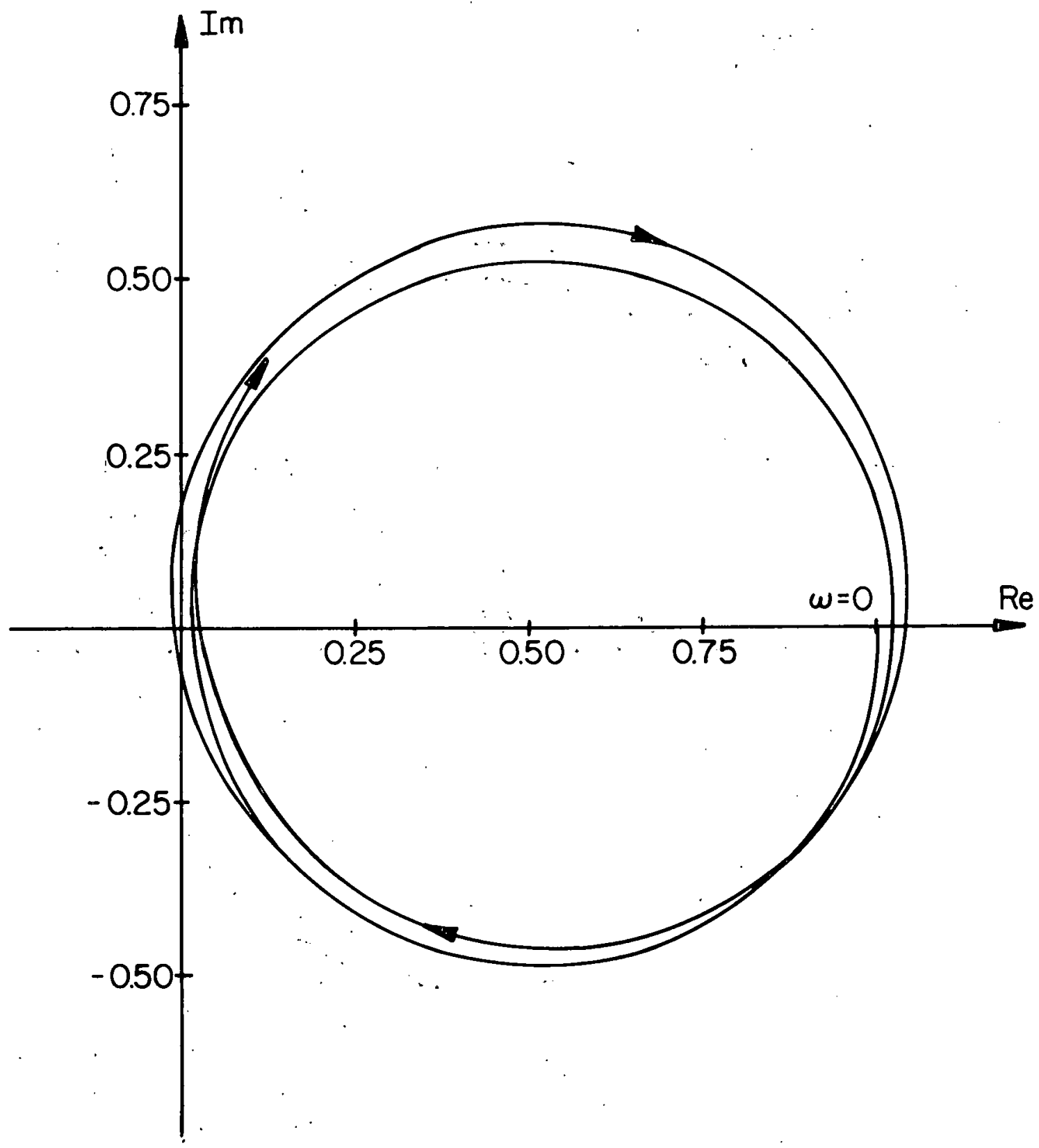

Figure 4.18. Modified Mikhailov Curve at RP-4. $\tau=375 \mathrm{sec}$. 
again past the critical value of 323 seconds (associated with $\omega_{2}$ ) brings one of the loops back to the right of the origin (Fig. 4.18), but the system remains unstable.

If the reference point were moved closer and closer to the stable first quadrant, more and more stability bands would develop. To illustrate this, consider the following information obtained at the point $(-0.0222,0.24)$ on Fig. 4.8 .

$$
\begin{aligned}
& \omega_{1}=2.592 \times 10^{-2} \sec ^{-1} \quad \omega_{2}=1.682 \times 10^{-2} \sec ^{-1} \\
& \tau_{10}=28.5 \mathrm{sec} \\
& \tau=271 \mathrm{sec} \\
& \tau_{12}^{11}=513 \mathrm{sec} \\
& \tau_{13}=756 \mathrm{sec} \\
& \tau_{20}=81.3 \mathrm{sec} \\
& \tau_{21}=455 \mathrm{sec} \\
& \tau_{22}=828 \mathrm{sec} \\
& \tau_{23}=1202 \mathrm{sec}
\end{aligned}
$$

From Eq. (4-54), the following stability bands are obtained.

$$
\begin{aligned}
& 0 \leqslant \tau \leqslant 28.5 \mathrm{sec} \quad \text { Stable } \\
& 28.5 \leqslant \tau \leqslant 81.3 \mathrm{sec} \quad \text { Unstable } \\
& 81.3 \leqslant \tau \leqslant 271 \mathrm{sec} \text { Stable } \\
& 281 \leqslant \tau \leqslant 455 \mathrm{sec} \quad \text { Unstable } \\
& 455 \leqslant \tau \leqslant 756 \mathrm{sec} \quad \text { Stable } \\
& \tau \geqslant 756 \text { sec } \quad \text { Unstable }
\end{aligned}
$$

From Eq. (4-55), we see that there are now three stability bands covering a larger range of delays rather than the one band at RP-4. 


\section{Some Comparisons}

In this section, we will give some rather cursory comparisons of the zero-delay, the infinite-delay, and the. Taylor series approxi-. mations to the exact analysis given in the previous section. Also, the possible validity of the approximations will be indicated.

The Zero-Delay Approximation

In Fig. 4.6, we have already seen the stability boundaries which resulted from the zero-delay approximation; and we have noted their relation to the exact analysis shown in Fig. 4.9. In these figures, it is obvious that the zero-delay approximation does not give a conservative estimate of the actual stability boundaries. It could therefore give erroneous predictions of stability. We have seen, however, that the zero-delay approximation may be used advantageously in conjunction with the analysis developed in Chapter 3.

The Infinite-Delay Approximation

By assuming that the time delays are extremely large, the system is effectively reduced to a fifth order model whose characteristic equation is the ordinary polynomial $Q(s)$. The thermionic reactor core then has a constant coolant-inlet temperature. To perform a Routh-Hurwitz analysis on this reduced model, a polynomial-multiplying computer program was developed to calculate the coefficients of the polynomials making up the first column of the Routh array. Then a standard polynomial-root-solving technique was used to plot the zeros of these polynomials in the parameter space $\left(\gamma_{1}, \gamma_{2}\right)$. It was found that within the physical range of $\gamma_{1}, \gamma_{2}$, only the constant term of $Q(s)$ was important. 
This condition is given by

$$
\gamma_{125} C_{25}+\gamma_{226} C_{26}=0
$$

Equation (4-56) is plotted in Fig. 4.19.

In comparing Fig. 4.19 with the exact analysis shown in Fig. 4.9, we see that the infinite-delay model does not give conservative stability predictions.

The Taylor Series Approximation

After approximating the delayed terms in Eq. $(4-31)$ by the twoterm Taylor seriés, the characteristic equation can be shown to be

$$
M(s)=Q(s)+R(s)\left[\xi_{C R} \xi_{R C} s^{2}-\left(\xi_{R C}+\xi_{C R}\right) s+1\right]
$$

where $Q(s)$ and $R(s)$ are given in Eqs. (4-42) and (4-43). From Eq. (4-57), it is obvious that the system stability no longer depends only on the sum of $\xi_{\mathrm{RC}}$ and $\xi_{\mathrm{CR}}$, but on their specific values as well. To give an indication of how well Eq. (4-57) approximates the delayed system stability, the critical values, $\tau_{\text {crit-f, were calculated }}$ at RP-I by a Routh-Hurwitz analysis of Eq. (4-57) and compared with the exact results previously shown in Fig. 4.15. The calculations were made for two choices of $\xi_{\mathrm{CR}}$ and $\xi_{\mathrm{RC}}$. For Case 1 , we let $\cdot \xi_{\mathrm{CR}}=\xi_{\mathrm{RC}}=\tau$; and for Case 2 we let $\tau=\xi_{\mathrm{CR}^{\mathrm{r}}}$ and $\xi_{\mathrm{RC}}=0$. The results of these two cases are shown along with the exact result in Fig. 4.20. For each of the three, the portion above the curve represents values leading to instability while those below indicate stable values. 


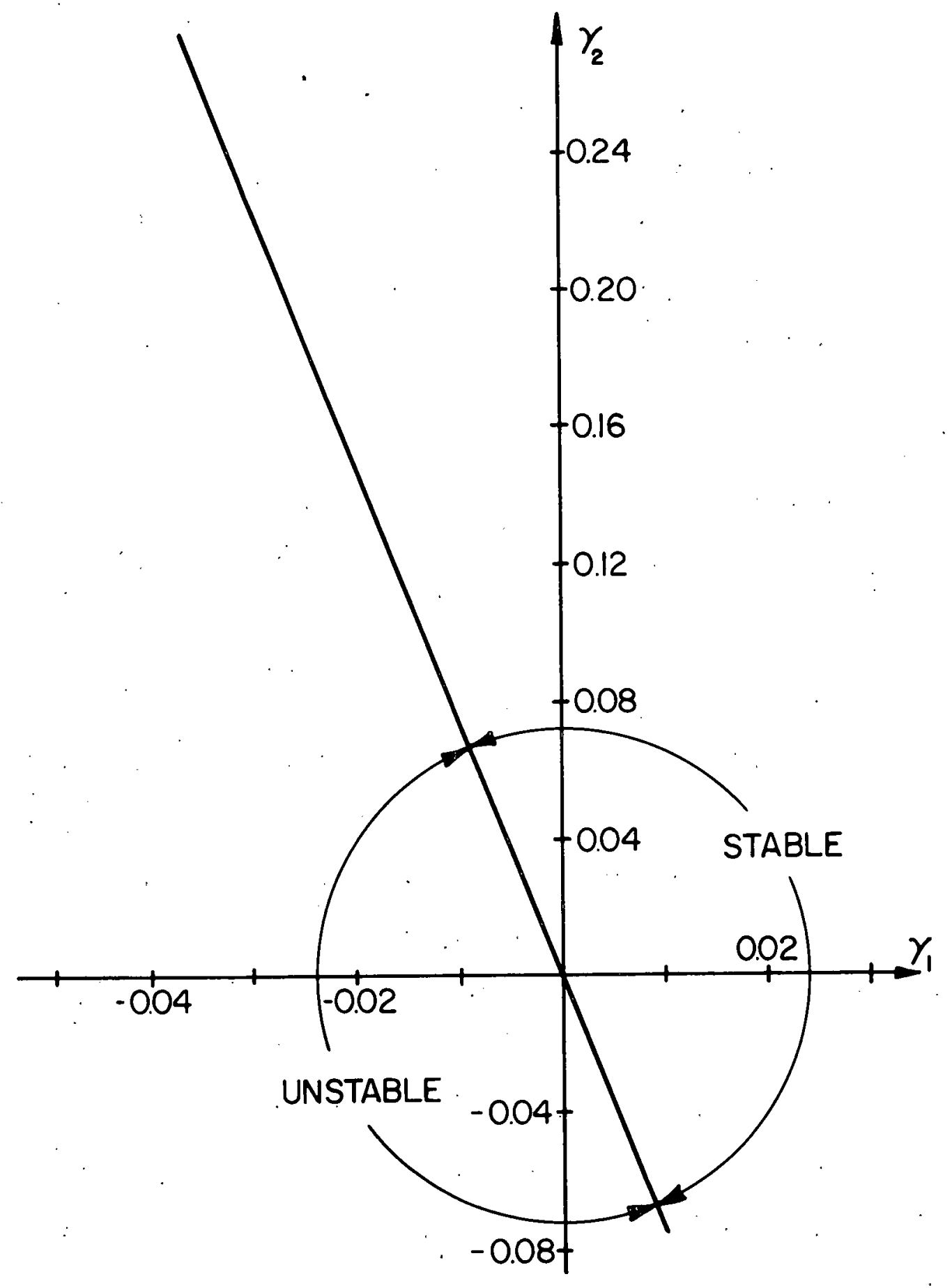

Figure 4.19. Infinite-Delay Stability Result 


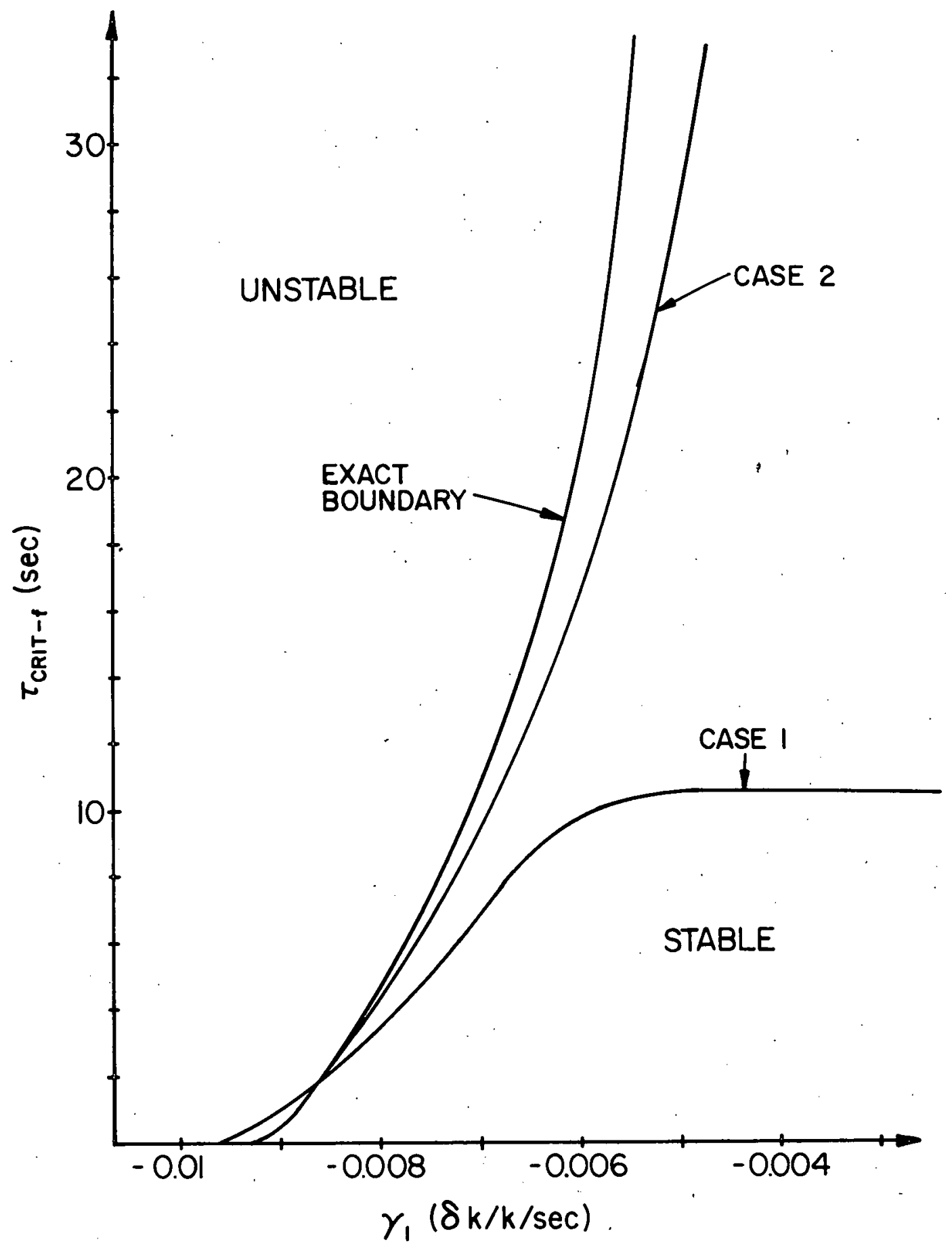

Figure 4.20. Comparison of Exact Analysis and Taylor Series Approximations at RP-1 
In Fig. 4.20, we see that for Case 1 the boundary closely approximates the exact curve over a partial range of $\gamma_{l}$, but then saturates at a value of 10.5 seconds. Further, we can note that, in a short interval, Case 1 gives a non-conservative stability prediction. Since the 10.5 second stability boundary extends into the stable first quadrant this case gives an extremely restrictive stability estimate of the conditional region.

Case 2 more closely approximates the exact boundary over the range shown, but still saturates at a value of $\tau_{\text {crit-f }}=3400$ seconds. From Cases 1 and 2, we can see that a Taylor series approximation can lead to non-conservative as well as highly restrictive stability predictions. As in Case 2, it is seen that in certain instances the Taylor series approximation can give good results in some cases, but the instances can not be determined nor assured prior to its utilization. Since, in addition, the Taylor series analysis is nearly as difficult as the exact treatment, it does not appear promising as an approximate technique for describing systems with pure time delays.

However, in using the Taylor series approximation, the appropriate question to be resolved is how well does the Taylor series approximation describe the physical processes to be modeled rather than how well it approximates the pure time delay model. The answer to this question will of course differ from system to system. 


\section{CHAPTER 5}

\section{CONCLUSIONS}

In the foregoing chapters, a method for analyzing the dependence of linear system stability on the system parameters has been developed for models containing pure time delays. Based on the Mikhailov stability criterion, the method identifies regions of the parameter space in which the system stability is conditional on the value of the time delay. In this identification, use is made of Déscartes' rule of signs and of Sturm's theorem. Combining the results of this analysis with the conventional Routh-Hurwitz analysis of the zero-delay approximation gives a complete linear stability description of the system parameter space.

The method was applied to a seventh-order, in-core thermionic reactor model containing two coolant time delays between the radiator and the core. Stability was found to depend on the sum of the two delays. The results of the analysis revealed the existence of conditional instabilities within physically possible operating regions of the parameter space.

The parametric stability analysis technique developed in this. work is probably best suited for. use in a preliminary stability analysis of a system whose model has been reduced to the essential description of the physical phenomena as in the case of the thermionic model. Since the results from this method can be obtained in a short period of time, 
and since it is possible to gain considerable insight into the effects of the time delays on system stability, the method would appear to be a highly useful approach in preliminary design studies of systems with time delays.

In the analysis of the seventh-order system, the major difficulty encountered was in the development of the characteristic equation. The development was done by hand; and, although quite ponderous even for the seventh-order system, it could probably be extended without change up to an order of about ten, provided the matrix A in Eq. (2-7) remained relatively sparse. Beyond this, however, the hand calculation would become impractical.

Therefore, the stability analysis of larger order systems with one constant delay hinges first on the ability to develop the coefficients of the characteristic equation as functions of the parameters which are under consideration. If these parameters are not numerous nor appear in too many elements of the characteristic determinant, it. would be feasible to develop a computer technique for accomplishing this result. Currently, computer methods are available which will calculate the numerical coefficients of the characteristic polynomial given the numerical elements of the characteristic determinant. By expanding the characteristic determinant into a specific sequence of minors, it is possible to bring all the exponentials and unspecified parameters into the coefficients of the remaining minors and to leave these minors in a form for which computer techniques already exists. A.l though compli- : cated, a technique such as this appears entirely feasible. 
For the seventh-order model, the stability analysis subsequent to the development of the characteristic polynomial made use of a digital computer to plot the stability zones in the parameter space. There would be no difficulty in extending these computer programs to analyze higher order systems up to about 15 . This is also true for the computer techniques used in the analysis of the zero-delay stability. Beyond 15, however, numerical search techniques would probably be necessary to increase the order of the system which could practicably be analyzed.

In Chapter 4, we saw that the infinite-delay, zero-delay, and Taylor series approximations can lead to erroneous as well as highly restrictive stability predictions. These approximations thus offer no real aid in attempting to analyze a larger order system.

A direct numerical solution to the system equations with delay presents difficulties in the regions of conditional stability. If one desires to determine the effect of the coolant lag on system stability, a number of delay intervals must be covered in order to obtain satisfactory results. Thus, one is limited to areas of the conditionally stable region in which the critical delays are manageably small. However, as we have seen, these occur near the unstable boundary of the conditional region meaning that the system has roots which are near the imaginary axis. In this case, solutions require a rather long period of time for the numerical results to indicate the desired properties. Several attempts at numerical solutions in these regions found computer times running at about $1 \frac{1}{2}$ times real time. 
In addition to overcoming some of these mentioned difficulties associated with the analysis of larger system models, several other extensions of this work are salient. The first extension would be to develop the capability of analyzing systems whose stability depends on several time delays rather than just one. This of course would complicate the analysis since Eq. (3-35) would no longer result in a polynomial upon squaring both sides. However, combining the requirements. given in Eq. (3-35) with those of Eq. (3-36) might lead to mutually exclusive ranges of $\omega_{k}$ which would thus simplify the analysis.. Another extension of this method would be to further investigate the dependence of stability on the time delays within the conditionally stable zones. One approach to this problem might be through Sturm's theorem by searching for positive, real roots of Eq. (3-35) within certain finite ranges rather than over the entire positive axis. Finally, a useful extension would be to find some suitable approximation whose stability zone predictions would be conservative relative to the exact zones and whose analysis would be more tractable than the exact approach. 
AP PENDIX A

DEFINITIONS OF SYMBOLS IN CHAPTER 4

$\begin{array}{ll}\mathrm{n} & \text { reactor power density }\left(\mathrm{W} / \mathrm{cm}^{3}\right) \\ \beta & \text { delayed neutron fraction } \\ \rho & \text { reactivity } \\ \lambda & \text { effective one-group decay constant }\left(\mathrm{sec}^{-1}\right)\end{array}$

$\lambda^{-1}=\beta^{-1} \sum_{i} \lambda_{i}^{-1} \lambda_{i}^{-1}$

$\alpha, \alpha_{2} \quad$ temperature coefficients of reactivity for regions one and two $\left({ }^{\circ} \mathrm{K}^{-1}\right)$

$\mathrm{T}_{1}, \mathrm{~T}_{2} \quad$ average temperatures of regions one and two $\left({ }^{\circ} \mathrm{K}\right)$

$\rho_{\mathrm{f}}, \rho_{\mathrm{e}}, \rho_{\mathrm{C}}, \rho_{\mathrm{cl}}, \rho_{\mathrm{C} 0}, \rho_{\mathrm{RP}} \quad$ densities of the fuel, emitter, collector, insulator, clad, coolant, and radiator pipes $\left(\mathrm{gm} / \mathrm{cm}^{3}\right)$

$V_{f}, V_{e}, V_{c}, V_{I}, V_{c I}, V_{c o c} \quad$ volumes of the fuel, emitter, collector, insulator, clad, and coolant associated with one diode $\left(\mathrm{cm}^{3}\right)$

$\mathrm{V}_{\mathrm{RP}}, \mathrm{V}_{\mathrm{COR}}$

volumes of radiator material and coolant associated with one radiator pipe $\left(\mathrm{cm}^{3}\right)$

$\mathrm{C}_{\mathrm{P}_{\mathrm{f}}}, \mathrm{C}_{\mathrm{p}_{\mathrm{e}}}, \mathrm{C}_{\mathrm{p}_{\mathrm{c}}}, \mathrm{C}_{\mathrm{p}_{I}}, \mathrm{C}_{\mathrm{p}_{\mathrm{cl}}}$

specific heats of the component materials (W-sec $/ \mathrm{gm}^{\circ} \mathrm{K}$ )

$\mathrm{C}_{\mathrm{p}_{\mathrm{CO}}, \mathrm{C}_{\mathrm{P}_{\mathrm{RP}}}}$

$T_{f}, T_{e}, T_{R P}$

average temperatures of the fuel

emitter and radiator material $\left({ }^{\circ} \mathrm{K}\right)$

$\mathrm{T}_{\text {coi }}, \mathrm{T}_{\text {coe }}, \mathrm{T}_{\text {co }}$

average coolant temperature associated with the core inlet, exit, and mean $\left({ }^{\circ} \mathrm{K}\right)$ 


$$
\begin{aligned}
& T_{R i}, T_{R e}, T_{R} \\
& k_{f}, k_{e}, k_{c}, k_{I}, k_{c} \\
& L_{f}, L_{e}, L_{c}, L_{I}, L_{c l} \\
& R_{f}, R_{e}, R_{c}, R_{I}, R_{c l} \\
& q_{e} \\
& T_{f}^{\prime}, T_{e}^{\prime} \\
& n_{e} \\
& N_{D S} \\
& N_{D P} \\
& N_{R P}
\end{aligned}
$$

$\mathrm{h}$

$\omega_{\operatorname{coc}}$

${ }^{\text {AN }}$

$\varepsilon_{r}$

$\sigma$

\section{$\mathrm{A}_{\mathrm{RS}}$}

0

tarm average coolant temperature associated with the radiator, inlet, exit, and average. $\left({ }^{\circ} \mathrm{K}\right)$

thermal conductivities of the component materials $\left(\mathrm{W} / \mathrm{cm}^{\circ} \mathrm{K}\right)$

lengths of components associated with one diode $(\mathrm{cm})$

outer component radii associated with one diode (See Fig. 4.1) (cm)

heat-loss rate from emitter surface $\left(W / \mathrm{cm}^{2}\right)$

fuel and emitter temperatures as functions of radial positions $\left({ }^{\circ} \mathrm{K}\right)$

diode efficiency

number of diodes in a diode stack

number of diode stacks in the core

number of radiator pipes

coolant convective heat-transfer coefficient, $\left(\mathrm{W} / \mathrm{cm}^{2}-{ }^{\circ} \mathrm{K}\right)$

coolant mass flow rate associated with one diode stack $(\mathrm{gm} / \mathrm{sec})$

inside surface area of one radiator pipe $\left(\mathrm{cm}^{2}\right)$

emissivity of radiator surface

Stefan-Boltzmann constant $\left(5.6699 \times 10^{-12}\right.$ $\left.\mathrm{W} / \mathrm{cm}^{2}-{ }^{\circ} \mathrm{K}^{4}\right)$

radiator surface area $\left(\mathrm{cm}^{2}\right)$

symbols followed by this subscript indicate that the steady state value is to be taken

radiator armor thickness $(\mathrm{cm})$ 


$$
\begin{aligned}
& R_{P I N} \\
& A_{R C} \\
& U_{C O C} \\
& U_{C O R}
\end{aligned}
$$

inside radius of radiator pipe $(\mathrm{cm})$

cross sectional flow area of radiator pipe $\left(\mathrm{cm}^{2}\right)$

flow velocity in diode stack $(\mathrm{cm} / \mathrm{sec})$

flow velocity in radiator pipe $(\mathrm{cm} / \mathrm{sec})$ 
THIS PAGE

WAS INTENTIONALLY LEFT BLANK 
APPENDIX B

DEFINITIONS OF $\boldsymbol{c}_{\mathbf{i}}$ CONSTANTS

$$
\begin{array}{ll}
c_{1}=n_{1}+n_{3}+n_{4}+n_{5} & c_{2}=n_{3}+n_{4}+n_{5} \\
c_{3}=n_{3} n_{5} & c_{4}=n_{2}\left(n_{3}-\lambda\right) \\
c_{5}=n_{2}\left(n_{3}-\lambda\right)\left(n_{4}+n_{5}\right) & c_{6}=n_{2} n_{3} n_{4} \\
c_{7}=n_{5}+2 n_{6}+n_{7} & c_{8}=n_{5} n_{6}+\left(n_{5}+n_{6}\right)\left(n_{6}+n_{7}\right) \\
c_{9}=n_{5} n_{6}\left(n_{6}+n_{7}\right) & c_{10}=c_{1}+c_{7} \\
c_{11}=c_{2}+\lambda+c_{7} & c_{12}=n_{12} c_{2}+c_{3}+c_{1} c_{7}+c_{8} \\
c_{13}=\lambda c_{2}+c_{3}+c_{7}\left(c_{2}+\lambda\right)+c_{8} & c_{14}=n_{2} c_{2}-c_{4}+n_{2} c_{7}
\end{array} .
$$

$$
\begin{aligned}
& c_{15}=n_{1} c_{3}+c_{2} c_{7} n_{1}+c_{3} c_{7}+c_{1} c_{8}+c_{9} \\
& c_{16}=\lambda c_{3}+c_{7} \lambda c_{2}+c_{3} c_{7}+c_{8}\left(c_{2}+\lambda\right)+c_{9} . \\
& c_{17}=c_{6}+n_{2} c_{3}-c_{5}+\eta_{2} c_{2} c_{7}+n_{2} c_{8}-c_{4} c_{7} \\
& c_{18}=\eta_{1} c_{3} c_{7}+\eta_{1} c_{2} c_{8}+c_{3} c_{8}+c_{1} c_{9} \\
& c_{19}=\lambda c_{3} c_{7}+\lambda c_{2} c_{8}+c_{3} c_{8}+c_{9}\left(c_{2}+\lambda\right) \\
& c_{20}=c_{6} c_{7}+\eta_{2} c_{3} c_{7}-c_{5} c_{7}+n_{2} c_{2} c_{8}-c_{4} c_{8}+\eta_{2} c_{9}
\end{aligned}
$$

111 
112

$$
\begin{aligned}
& \mathrm{C}_{21}=\eta_{1} \mathrm{C}_{3} \mathrm{C}_{8}+\mathrm{n}_{1} \mathrm{C}_{2} \mathrm{C}_{9}+\mathrm{C}_{3} \mathrm{C}_{9} \\
& C_{22}=\lambda C_{3} C_{8}+\lambda C_{2} C_{9}+C_{3} C_{9} \text {. } \\
& c_{23}=c_{6} c_{8}+\eta_{2} c_{3} c_{8}-c_{5} c_{8}+\eta_{2} c_{2} c_{9}-c_{4} c_{9} \\
& c_{24}=n_{1} C_{3} C_{9} \\
& \mathrm{C}_{25}=\lambda \mathrm{C}_{3} \mathrm{C}_{9} \\
& C_{26}=C_{6} C_{9}+\eta_{2} C_{3} C_{9}-C_{5} C_{9} \\
& c_{27}=n_{5} n_{6}\left(n_{6}-n_{7}\right)\left(n_{4}-n_{5}\right) \\
& c_{28}=n_{4}\left(n_{6}-n_{7}\right) n_{5} n_{6} \\
& c_{29}=c_{27}\left(n_{1}+n_{3}\right)-c_{28} n_{3} \text {. } \\
& c_{30}=C_{27}\left(\lambda+\eta_{3}\right)-C_{28} n_{3} \\
& C_{31}=C_{27} n_{2} \\
& c_{32}=n_{1} n_{3}\left(c_{27}-c_{28}\right) \\
& c_{33}=\left(C_{27}-C_{28}\right) n_{3} \lambda \\
& \mathrm{C}_{34}^{\cdot}=n_{2} \lambda \mathrm{C}_{27}
\end{aligned}
$$




\section{APPENDIX C}

\section{DEFINITIONS OF $\mathrm{F}_{i}$ CONSTANTS}

$$
\begin{array}{ll}
F_{1}=2\left(C_{10}-C_{11}\right) & F_{2}=-2 n_{2} \\
F_{3}=C_{10}^{2}-2 C_{12} & F_{4}=C_{11}^{2}-2 C_{13} \\
F_{5}=2 C_{11} n_{2}-2 C_{14} & F_{6}=n_{2}^{2} . \\
F_{7}=2\left(C_{11} C_{12}-C_{15}-C_{10} C_{13}+C_{16}\right) & F_{8}=2\left(n_{2} C_{12}-C_{10} C_{14}+C_{17}\right) \\
F_{9}=C_{12}^{2}-2 C_{10} C_{15}+2 C_{18} & F_{10}=C_{13}^{2}+2 C_{19}-2 C_{11} C_{16}
\end{array}
$$

$$
\begin{aligned}
& \mathrm{F}_{11}=2\left(\mathrm{C}_{13} \mathrm{C}_{14}+\mathrm{C}_{20}-\mathrm{C}_{11} \mathrm{C}_{17}-2 \mathrm{n}_{2} \mathrm{C}_{16}\right) \\
& F_{12}=C_{14}^{2}-2 n_{2} C_{17} \\
& F_{13}=2\left(C_{13} C_{15}+C_{21}+C_{10} C_{19}-C_{22}-C_{11} C_{18}-C_{12} C_{16}\right) \\
& \mathrm{F}_{14}=2\left(\mathrm{C}_{14} \mathrm{C}_{15}+\mathrm{C}_{10} \mathrm{C}_{20}-\mathrm{C}_{23}-\mathrm{n}_{2} \mathrm{C}_{18}-\mathrm{C}_{12} \mathrm{C}_{17}\right) \\
& \mathrm{F}_{15}=\mathrm{C}_{15}^{2}-2 \mathrm{C}_{24}+2 \mathrm{C}_{10} \mathrm{C}_{21}-2 \mathrm{C}_{12} \mathrm{C}_{18} \\
& F_{16}=C_{16}^{2}-2 C_{25}-2 C_{13} C_{19}+2 C_{11} C_{22} \\
& \mathrm{~F}_{17}=2\left(\mathrm{C}_{16} \mathrm{C}_{17}-\mathrm{C}_{26}-\mathrm{C}_{13} \mathrm{C}_{20}-\mathrm{C}_{14} \mathrm{C}_{19}+\mathrm{C}_{11} \mathrm{C}_{23}+n_{2} \mathrm{C}_{22}\right) \\
& F_{18}=C_{17}^{2}-2 C_{14} C_{20}+2 \eta_{2} c_{23} \\
& F_{19}=2\left(C_{16} C_{18}-C_{10} C_{25}-C_{13} C_{21}-C_{15} C_{19}+C_{11} C_{24}+C_{12} C_{22}\right) \\
& F_{20}=2\left(C_{17} C_{18}-C_{10} C_{26}-C_{14} C_{21}-C_{15} C_{20}+n_{2} C_{24}+C_{12} C_{23}\right) \\
& F_{21}=C_{18}^{2}-2 C_{15} C_{21}+2 C_{12} C_{24}-C_{27}^{2}
\end{aligned}
$$


114

$$
\begin{aligned}
& F_{22}=C_{19}^{2}+2 C_{13} C_{25}-2 C_{16} C_{22}-C_{27}^{2} \\
& F_{23}=2\left(C_{19} C_{20}+C_{13} C_{26}+C_{14} C_{25}-C_{16} C_{23}-C_{17} C_{22}\right) \\
& \mathrm{F}_{24}=\mathrm{C}_{20}^{2}+2\left(\mathrm{C}_{14} \mathrm{C}_{26}-\mathrm{C}_{17} \mathrm{C}_{23}\right) \\
& \mathrm{F}_{25}=2\left(\mathrm{C}_{19} \mathrm{C}_{21}+\mathrm{C}_{15} \mathrm{C}_{25}-\mathrm{C}_{16} \mathrm{C}_{24}-\mathrm{C}_{18} \mathrm{C}_{22}-\mathrm{C}_{27} \mathrm{C}_{29}+\mathrm{C}_{27} \mathrm{C}_{30}\right) \\
& \mathrm{F}_{26}=2\left(\mathrm{C}_{20} \mathrm{C}_{21}+\mathrm{C}_{15} \mathrm{C}_{26}-\mathrm{C}_{17} \mathrm{C}_{24}-\mathrm{C}_{18} \mathrm{C}_{23}+\mathrm{C}_{27} \mathrm{C}_{31}\right) \\
& \mathrm{F}_{27}=\mathrm{C}_{21}^{2}-2 \mathrm{C}_{18} \mathrm{C}_{24}-\mathrm{C}_{29}^{2}+2 \mathrm{C}_{27} \mathrm{C}_{32} \\
& F_{28}=C_{22}^{2}-2 C_{19} C_{25}-C_{30}^{2}+2 C_{27} C_{33} \\
& \mathrm{~F}_{29}=2\left(\mathrm{C}_{22} \mathrm{C}_{23}-\mathrm{C}_{19} \mathrm{C}_{26}-\mathrm{C}_{20} \mathrm{C}_{25}-\mathrm{C}_{30} \mathrm{C}_{31}+\mathrm{C}_{27} \mathrm{C}_{34}\right) \\
& \mathrm{F}_{30}=\mathrm{C}_{23}^{2}-2 \mathrm{C}_{20} \mathrm{C}_{26}-\mathrm{C}_{31}^{2} \\
& \mathrm{~F}_{31}=2\left(\mathrm{C}_{22} \mathrm{C}_{24}-\mathrm{C}_{21} \mathrm{C}_{25}-\mathrm{C}_{30} \mathrm{C}_{32}+\mathrm{C}_{29} \mathrm{C}_{33}\right) \\
& \mathrm{F}_{32}=2\left(\mathrm{C}_{23} \mathrm{C}_{24}-\mathrm{C}_{21} \mathrm{C}_{26}-\mathrm{C}_{31} \mathrm{C}_{32}+\mathrm{C}_{29} \mathrm{C}_{34}\right) \\
& \mathrm{F}_{33}=\mathrm{C}_{24}^{2}-\mathrm{C}_{32}^{2} \\
& F_{34}=C_{25}^{2}-C_{33}^{2} \text {. } \\
& F_{35}=2\left(C_{25} C_{26}-C_{33} C_{34}\right) \\
& F_{36}=\dot{C}_{26}^{2}-C_{34}^{2}
\end{aligned}
$$


Archbold, J. W., Algebra, Sir Isaac Pitman \& Sons, Ltd., London (1964).

Ball, S. J., "Approximate Models for Distributed-Parameter Heat Transfer Systems," Instru. Soc. of Amer. Trans., 3, 38 (1964).

Ba11, S. J., "Nuclear Desalination Dual-Purpose Plant Control Interim Report Appendix," USAEC Report ORNL-TM-1618, Part II (January 1967).

Bellman, Richard, and Kenneth L. Cooke, Differential-Difference Equations, Academic Press, New York (1963).

Brehm, R. L., D. L. Hetrick, J. G. Guppy, K. D. Kearns, and T. R. Schmidt, "Dynamics of a Fast Reactor With In-Core Thermionic Converters, Second. Annual Report," Engineering Experiment Station, University of Arizona, Tucson, Arizona (1968).

Brehm, R. L., D. L. Hetrick and T. R. Schmidt, "Stability Studies of In-Core Thermionic Reactor Concepts," Nuc. Appl. and Tech., 7 117 (1969).

Buck, R. Creighton, Advanced Calculus, McGraw-Hill Book Co., Inc., New York (1956).

Chezem, C. G., and R. L. Seale, "Temperature-Dependent Kinetics of Coupled-Core Nuclear Reactor Systems," NASA Contractor Report NASA-CR-447 (May 1964).

Chezem, C. G., and W. H. Köhler, eds., Coupled-Core Reactor Kinetics, Proc., The Texas A. \& M. Press (January 1967).

Churchill, Ruel V., Complex Variables and Applications, McGraw-Hill Book Co., Inc., New York (1960).

DePree, John D., and Charles C. Oehring, Elements of Complex Analysis, Addison-Wesley Publishing Co., Reading, Mass. (1969).

Devooght, J., and H. B. Smets, "Determination of Stability Domains in Point Reactor Dynamics," Nuc. Sci. Eng., 28, 226 (1967).

Driver, Rodney D., "Existence and Stability of Solutions of a DelayDifferential System," Archive for Rational Mechanics and Analysis, 16, 401 (1962). 
El'sgol'ts, L. E., Qualitative Methods in Mathematical Analysis, Translations of Math. Monographs, Amer. Math. Soc., Providence, R. I. (1964).

El'sgol'ts, L. E., Introduction to the Theory of Differential Equations With Deviating Arguments, Holden-Day, Inc., San Francisco (1966).

Forbes, S. G., "Quarterly Progress Report, Reactor Projects Branch," G. O. Bright, ed., USAEC Report IDO-16452 p. 38; (August 1958).

Gage, Stephen J.; F. T. Adler, and P. N. Powers, "Investigations on Nonlinear Stability of Coupled Nuclear Systems," in Neutron Dynamics and Control, Proc. of Symposium, D. L. Hetrick and L. E. Weaver, eds., USAEC Division of Technical Information (1966).

Gronroos, H., "Analog Studies of Thermionic Reactor Dynamics," SPS37-45 Vo1. IV, pp. 151-162, Jet Propulsion Laboratory (1967).

Guppy, J. G., "Analytical and Experimental Studies Relating to the Simulated Start-Up of In=Core Thermionic Reactor Systems," Ph.D. Dissertation, University of Arizona, Tucson (1970a).

Guppy, J. G., Private Communication (1970b).

Heath, C. A., "Possible Instabilities in a Thermionic Reactor With All Negative Reactivity Coefficients," IEEE 1968 Thermionic Specialist Conference, p. 342 (October 1968).

Householder, Alton S., The Theory of Matrices in Numerical Analysis, Blaisdell Publishing Co., New York (1964).

Köhler, W. H., "The Effect of Short Delay Times in Super-PromptCritical Excursions of Fast Reactors," Trans. ANS, 12, 735 (1969).

Krasovskii, N. N., Stability of Motion, Stanford University Press, Stanford, California (1963).

Lehnigk, Siegfried H., Stability Theorems for Linear Motions, Prenticehall, Inc., Englewood Cliffs, N. J. (1966).

Margolis, S. G., and W. G. Vogt, "Control Engineering Applications of V. I. Zubov's Construction Procedure for Liapunov Functions" IEEE Trans. on Automatic Control, 8, 104 (1963).

Murray, H. S., and L. E. Weaver, "Stability of Coupled-Core Nuclear Reactor Systems," NASA Contractor Report NASA-CR-447 (May 1966). 
Pinney, Edmund, Ordinary Difference-Differential Equations, University of California Press, Berkeley and Los Angeles (1959).

Pontryagin, L. S., "On Zeros of Some Elementary Transcendental Functions," Amer. Math. Soc. Translations, Ser. 2, 1, 95 (1955).

Popov, E. P., The Dynamics of Automatic Control Systems, AddisonWesley Publishing Co., Inc., Reading, Mașs. (1962).

Razumikhin, B. S., "The Application of Liapunov's Method to Problems . in the Stability of a System with Delay," Automation and Remote Control, 21, 515 (1960).

Rodden, J. J., "Numerical Applications of Liapunov's Stability Theory," Proc. 1964 Joint Automatic Control Conf., Stanford, 5, 261 (1964).

Thompson, T. J., and J. G. Beckerly, eds., The Technology of Nuclear Reactor Safety, Vol. 1, The M.I.T. Press (1964).

Truxal, John G., Control System Synthesis, McGraw-Hill Book Co., Inc., New York (1955).

Weaver, Lynn E., System Analysis of Nuclear Reactor Dynamics, Rowman and Littlefield, Inc., New York (1963).

Wilkins, D. R., "SIMCON - A Digital Computer Program for Computing Thermionic Converter Performance Characteristics," GESR-2109, (February 1968).

Wright, W. C., and T. W. Kerlin, "An Efficient, Computer-Oriented Method for Stability Analysis of Large Multivariable Systems," Univ. of Tennessee Nuc. Eng. Dept. Report NEUT-2806-3 (July 1968).

Wright, W. C., and T. W. Kerlin, "The Modified Mikhailov Method for Reactor Stability Analysis," Trans. ANS 12, 736 (1969). 